\title{
Impression 4D : Une cécité de confort et/ou une approche systémique empêchée ?
}

\section{D Printing: Comfort blindness and/or a systemic approach prohibited?}

\author{
Frédéric Demoly ${ }^{1}$, Jean-Claude André ${ }^{2}$ \\ ${ }^{1}$ ICB UMR 6303 CNRS, Université de Bourgogne Franche-Comté, UTBM, Rue du Château - 90010 Belfort Cedex - \\ France, Frederic.demoly@utbm.fr \\ ${ }^{2}$ LRGP - UMR7274 CNRS-UL, 1, rue Grandville, 54000 Nancy - France, jean-claude.andre@univ-morraine.fr
}

\begin{abstract}
RÉSUMÉ. En ajoutant de possibles changements temporels de forme et/ou de fonctionnalité à la fabrication additive, l'impression 4D apporte des potentiels nouveaux à ce domaine jeune encore en pleine expansion. Si des promesses transgressives sont associées à cette technologie à fort potentiel de croissance en recherche académique, les applications ciblées sont loin d'être atteintes. Sur la base de ce bilan, il apparait que des domaines proches, en situation de possible porosité disciplinaire, peuvent, avec un peu de créativité, faire quitter les travaux fortement autocentrés actuels pour tenter de ressourcer l'impression 4D, pour autant que la prise de risque en recherche interdisciplinaire soit mieux soutenue. Une proposition de « feuille de route » probablement améliorable est proposée : elle concerne d'une part, un axe scientifique où l'activité 4D recherchée s'appuie sur des couplages originaux entre matériaux (dont les méta-matériaux) et procédés, des fabrications hybrides multi-matériaux, un axe organisationnel où l'interdisciplinarité et la prise de risque seraient privilégiées.
\end{abstract}

ABSTRACT. By adding possible temporal changes of form and/or functionality to additive manufacturing, 4D printing brings new potentials to this still expanding new field. While there is transgressive promise associated with this technology with high growth potential in academic research, targeted applications are far from being achieved. On the basis of this assessment, it appears that related fields, in a situation of possible disciplinary porosity, can, with some creativity, move away from the current highly self-centered work to try to re-invigorate $4 \mathrm{D}$ printing, provided that the risk-taking in interdisciplinary research is better supported. A proposal for a "roadmap" that is likely to be improved is proposed: it concerns on the one hand, a scientific axis where the 4D activity researched relies on original couplings between materials (including meta-materials) and processes, hybrid multi-material fabrications, and on the other hand, an organizational axis where interdisciplinarity and risk-taking would be privileged.

MOTS-CLÉS. Fabrication additive, impression 3D/4D, créativité, interdisciplinarité, rupture technologique, applications industrielles.

KEYWORDS. Additive manufacturing, 3D/4D printing, creativity, interdisciplinarity, technological breakthrough, industrial applications.

«La caresse transcende le sensible. Non pas qu'elle aille au-delà du senti, plus loin que les sens, qu'elle se saisisse d'une nourriture sublime, tout en conservant, dans sa relation avec ce senti ultime, une intention de faim, qui va sur la nourriture qui se promet et se donne à cette faim, la creuse, comme si la caresse se nourrissait de sa propre faim. La caresse consiste à ne se saisir de rien, à solliciter ce qui s'échappe sans cesse de sa forme vers un avenir à solliciter, ce qui se dérobe comme s'il n'était pas encore. Ce n'est pas une intentionnalité de dévoilement, mais de recherche : marche à l'invisible » (Levinas, 1990).

\section{Introduction}

«Par quoi croyez-vous qu'Edison commence ? Par la fin, bien évidemment. Il met avec application la charrue avant les bœufs. Pourquoi se lancer en effet dans une entreprise difficile si personne n'y croit » (Akrich, Callon et Latour, 1988). Quand l'opinion est assez mûre, Edison lève le voile sur ses inventions, qui sont alors co-construites, adaptées, puis adoptées... De plus en plus, dans les réponses aux appels à propositions, les chercheurs jouent sur la promesse, parce que le jugement a priori est de règle... C'est vrai qu'il existe de nombreuses sources d'incertitudes, tout 
d'abord d'être considéré et financé pour réaliser des systèmes technologiques qui n'existent pas encore, de maîtriser le comportement individuel des futurs utilisateurs, de tenir compte de l'interaction des technologies avec des systèmes naturels et artificiels complexes, etc. (Hanson, 2010). Mais qu'en est-il pour l'impression 4D, présentée schématiquement sur la figure 1 ? C'est tout l'enjeu de cet article jouant entre connaissances scientifiques et techniques et environnement de la recherche et du transfert à la société, en allant des sciences «dures » aux sciences plus douces (et réciproquement)!

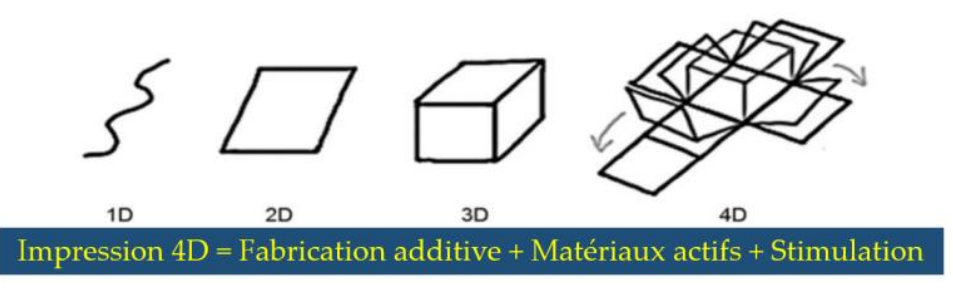

Figure 1. Impression $4 D$

Un domaine nouveau correspond à l'application d'une certaine vision sensible du monde à un futur scientifique et/ou technologique, noyau de création de voies de recherches inscrites souvent dans des «grammaires » que d'aucuns appellent paradigmes, qui s'appuient sur des intuitions primordiales qui font sens. Après cette gratuité originelle de l'idée heureuse, réel acte d'expression d'une liberté libérée, se créent des voies d'accomplissement visant une certaine « rentabilité », à l'intérieur de la morphologie des cultures et des organisations d'encadrements scientifiques. C'est à ce stade que le nouveau s'intègre dans des savoirs acquis exploités et développés par des communautés scientifiques, techniques et économiques. L'utilitarisme recherché par certains qui en sont issus exerce alors des influences (promesses, savoirs nouveaux, publications, etc.) jusqu'à la manifestation évidente de la dévitalisation du domaine, alors qu'un corps constitué de détenteurs de savoirs préalablement acceptés empêche un possible « dépôt de bilan » de la singularité validée initialement, avec une absence de volonté de soutenir des remises en causes, de favoriser la divergence ou la transgression et la prise de risques. « Entre expansion initiale et régression finale, la force créatrice s'abolit dans l'immobilisme critique; la puissance conquérante dans l'espace déliquescent ; et l'esprit de domination dans la décomposition spirituelle » (Colosimo, 2018)...

Or, les chercheurs du monde académique vivent entre pairs et sont, pour l'essentiel, financés sur programmes. Avec un tel pilotage formaté, il est sans doute difficile d'inculquer une culture de la remise en cause. "Et pour cause, l'évolution de leur carrière se construit sur le nombre de publications scientifiques qu'ils réalisent et le classement des journaux scientifiques publiant » (Nguyen, 2020). Or, entreprendre et s'engager dans des secteurs trop peu connus, aller vers du disruptif ou même simplement sortir de sa discipline sont autant d'investissements qui empêchent ou retardent le chercheur dans la rédaction d'articles, piliers de la carrière et du rayonnement quantitatif. Personne ne choisit volontairement d'échouer.

«Pour produire du nouveau, il faut certes «douter de...». Mais aussi pouvoir «se douter que... », c'est-à-dire accepter, au moins provisoirement, une connaissance mal étayée, fragile et provisoire. Par-delà le doute, c'est le risque de la pensée qu'il faut assumer » (Lévy-Leblond, 2020).

La créativité est une façon de penser. Il n'y a pas d'intitulé de poste dans le monde académique, ni un rôle au sommet de la hiérarchie parce qu'elle devrait, pour être effective, être intégrée dans la culture des unités de recherche, valable pour l'ensemble des personnels. Il ne peut y avoir en local de prétendu responsable des idées à mettre en œuvre parce qu'il n'y aurait pas appropriation aisée de ces dernières. Dans les faits, un climat propice à l'échange d'idées n'existe pratiquement pas, ce qui peut conduire à se déresponsabiliser et de rester pour des raisons de confort (mais de bonne qualité 
scientifique) dans la réponse à des appels d'offre convenus, où la rupture n'est pas recherchée (Lovric et Schneider, 2020). Par habitude, les chercheurs ne se sentent ainsi plus trop concernés par des opérations risquées, bien que l'idée leur plaise. Ils changent probablement d'avis lorsqu'ils rentrent dans les détails, étudient les avantages et les faiblesses à court terme d'opérations en rupture. Le principal obstacle n'est peut-être pas dans l'émergence d'idées, mais plutôt de conviction de l'intérêt de l'engagement dans ces idées pour les concrétiser. Pour Yves Michaud, cité par Atlantico (2020) « [L'] industrialisation de la science entraîne une bureaucratisation des programmes (la lutte pour les financements avec la question permanente des feed-back et de l'évaluation), pas mal de rigidité, mais aussi des phénomènes plus complexes. La communication permanente entre chercheurs et la compétition produisent des déplacements rapides vers les secteurs prometteurs ». Pour Jean-Michel Besnier, cité dans la même référence : «Voyez [...] le constat déprimant qui établit que l'effort de recherche consenti par nos sociétés croît tandis que la recherche de productivité décroît - constat, en d'autres termes, que la production des idées par nos chercheurs n'offre plus le retour sur investissement que l'on en espérait » (voir également Bloom et al, 2020).

Alors, les questions suivantes doivent être posées : Comment amener les chercheurs à faire émerger et à soutenir de nouvelles idées, en sachant qu'elles pourraient échouer ? Comment soutenir, en temps de crise et/ou d'indécision de possibles bifurcations? Des sorties des déterminismes ordinaires et des explorations de la complexité du monde ? A-t-on encore le droit de se tromper? Et surtout, l'erreur sert-elle à quelque chose ? Peut-on sortir des biais "d'homophilie » avec des évaluations plus favorables pour les tenants d'une théorie ou d'une école de pensée proches de celle des évaluateurs ? (AFIS, 2020). Alors, comment sortir de la conception d'objets fictionnels, devenue une fin en soi ? Etc. Comme le souligne Mercier (2020) : «Et n'est-ce pas aussi une des missions du chercheur d'élever le niveau global de curiosité, de surprendre et de transmettre un désir de science, une vocation »?

«Un type d'argument qui vous avertit que, si vous franchissez une première étape, vous vous retrouvez entraîné dans une suite de conséquences desquelles vous serez incapable de vous dégager et que, à la fin, vous roulerez de plus en plus vite, vers quelque résultat final désastreux » (Walton, 1992).

Peut-on appliquer ces considérations un peu morbides et fortement négatives à l'impression 4D, issue en 2013 de la « vieille » fabrication additive (premier brevet en 1984 par André, Le Méhauté et de Witte...) ? Cette base réflexive est partiellement engagée par André (2020 b). Selon Tibbits (2013) «Une révolution sans précédent est en cours. Il s'agit de la capacité de programmer des matériaux biologiques et physiques afin qu'ils changent de forme, changent de propriétés [...]. L'idée derrière l'impression $4 \mathrm{D}$ est de réaliser l'impression 3D avec des matériaux multiples, afin de pouvoir y mettre plusieurs matériaux, et d'y ajouter une nouvelle capacité : la transformation. Dès qu'elles sortent, les pièces peuvent se transformer d'une forme à une autre, directement, toutes seules. C'est comme de la robotique sans fils ni moteurs. Vous imprimez cette pièce, et elle peut se transformer en autre chose ». Qui n'en a pas rêvé après avoir le film «Terminator » ? Tibbits (2013 a) définit ainsi un objectif nouveau à l'impression 3D avec, en anticipation, son inclusion dans le contexte d'Industrie 4.0 (Jardim-Goncalves, Romero et Grilo, 2017; André, 2019), avec l'expression d'un besoin nouveau, parce que l'on ajoute un degré de liberté à la fabrication 3D.

\section{REMARQUE : fabrication additive}

La fabrication additive naît avec un principe de transformation localisée de la matière l'élément minimal permettant la description de cette transformation (liquide/solide poudre/solide ; etc.) est, par analogie au pixel, appelé voxel. Quand une surface a été façonnée, une nouvelle couche ou un autre ajout de matière), jointive avec la précédente, est à nouveau transformée, etc. De proche en proche, l'objet est ainsi construit. 
Or, avec l'Industrie 4.0, concept introduit en 2013 par Kagermann, Wahlster et Helbig, c'est tout le paradigme de la stratégie industrielle qui est transformé par le numérique, y compris sa logique de performance économique et les mécanismes de création de valeur qui la sous-tendent. En réduisant le coût de la complexité portée par la composante numérique d'un produit ou d'une machine à base standardisée, la fabrication additive 3D s'inscrit déjà dans cette logique. Mais, avec une opportunité complémentaire, qui est de disposer de possibilités originales liées à l'évolution résolue dans le temps et dans l'espace de la forme et de la fonctionnalité des objets créés, l'impression 4D peut disposer d'atouts spécifiques pour mieux coopérer avec les autres briques élémentaires de l'Industrie 4.0. Pour rappel, ce sont, hors Intelligence Artificielle, la robotique (souple) et l'Internet des objets (IoT). Le développement de l'axe spécifique constitué par l'impression 4D peut donc être un élément stratégique important d'intégration dans le progrès technique associé au concept d'Industrie 4.0. Par conception, la fabrication 4D génère une inséparabilité technologique lorsqu'il s'agit de produire une pièce en 3D dans l'imprimante, rendue active par des stimuli, plutôt que par des activités organisées en différentes étapes successives. Il s'agit donc d'une intégration perturbatrice majeure qui peut révolutionner le domaine 4.0, si elle peut satisfaire aux spécifications industrielles.

D'une part, l'impression 4D est récente et le nombre de publications (quelques centaines) encore accessible à une lecture (au moins partielle) de la part des auteurs. Il est donc possible, à partir de connaissances acquises en impression 3D, d'être un observateur de l'évolution d'un domaine émergent à fort contenu technologique. D'autre part, Tibbits en 2013 a placé avec détermination sur l'échiquier scientifique l'impression 4D comme une promesse importante avec des potentialités d'applications nombreuses en réparation médicales (prothèses et orthèses), dans les transports, dans la domotique, la robotique, etc., domaines applicatifs et/ou contributifs au concept d'Industrie 4.0.

Les auteurs, impliqués dans des recherches sur les thèmes 3D/4D ne vivent pas comme des ascètes hors du monde et intellectuellement radicaux, mais ont juste souhaité être des observateurs attentifs, curieux et responsable d'un domaine émergent dans lequel ils investissent beaucoup de leur temps. En ce sens, ils sont juges et parties prenante dans cette opération! C'est probable, leur exigence de douter, de se remettre en cause de manière aussi professionnelle que possible peut entrainer des rejets de la part de leur communauté scientifique considérant qu'il ne s'agit que d' " extravagantes suppositions » (ce que dénonçait déjà Descartes dans un cadre plus philosophique pour les réfuter (Descartes, 1990)). Cette situation d'alerte, alors que tous les voyants sont apparemment au vert sur la 4D, impose une discipline la plus exigeante possible, avec le risque que les propositions soient considérées comme cruelles parce qu'elles font sortir l'impression 4D de ses domaines actuels de confort. Toutefois, il existe des voies de ressourcement et le risque d'angoisse de l'anéantissement autour du sujet est limité à l'obligation de penser de manière moins suiviste, mais plus agile et plus responsable les recherches du domaine...

« L'Histoire alimente l'Histoire » (Battu, 2018) et il est peut être tôt pour que la clarté logique du développement de l'impression 4D sorte d'un certain flou, celui-ci ne pouvant apparaître qu'avec le plus recul possible. Mais, sept ans, c'est court... Cependant, nous avons pris le risque de tenter de tracer des liens qui unissent le passé récent au présent et de lancer des mises en garde sur les difficultés d'activités associées à des promesses déjà extraordinaires en 2013. Sur ces constats, il nous a paru intéressant d'examiner comment on peut passer d'une idée apparemment pertinente et surtout spectaculaire à un devenir, le plus légitime possible, avec ses réussites et ses échecs. C'est tout l'enjeu de cet article, qui ne doit pas être considéré comme une fin en soi, mais comme une ouverture sur un monde qui est encore à découvrir.

«La condition éleucratique [...] serait accordée, toute nationalité confondue, à ceux qui assument de considérer comme, au-dessus de toute forme de sacralité, la liberté de penser, la leur et celle des autres » (Ménissier, 2020). 
«Il y a, à l'intérieur des institutions de l'enseignement supérieur et de la recherche, une inévitable compétition pour des ressources matérielles et humaines limitées. Dans cette compétition, chacun tend à mettre son programme en valeur en présentant de simples espérances dans des promesses, voire, comme des résultats, et en jetant la suspicion sur les programmes concurrents » (Sperber, 1996).

L'histoire des grandes ruptures technologiques montre qu'au-delà de la découverte de nouveaux procédés, ce sont bien davantage les changements que celles-ci ont induits, par contamination croisée, à la fois dans les modes de production/consommation, les relations de travail, les moyens de transport et de communication, qui ont déterminé ces révolutions (cf. électricité, électronique, internet, GPS, etc.). Toutefois, après 36 ans depuis le premier brevet, l'innovation liée à la fabrication additive n'a pas reconfiguré le marché dans sa globalité, mais a changé des règles et des découpages obligeant des industriels à se réinventer (Zerbib et Mamavi, 2020). L'envie d'apporter sa petite pierre pour changer le monde nous tenaille tous parce que cela correspond à une valeur ajoutée personnelle, au témoignage d'une réalisation et c'est un sens au temps de présence sur Terre.

«Excité par son projet, sa première démarche pratique [celle du bricoleur] est pourtant rétrospective : il doit se retourner vers un ensemble déjà constitué, formé d'outils et de matériaux ; en faire ou en refaire l'inventaire ; enfin et surtout engager avec lui une sorte de dialogue, pour répertorier, avant de choisir entre elles, les réponses possibles que l'ensemble peut offrir au problème qu'il lui pose. Tous ces objets hétéroclites constituent son trésor, il les interroge pour comprendre ce que chacun d'eux pourrait " signifier », contribuant ainsi à définir un ensemble à réaliser, mais qui ne différera finalement de l'ensemble instrumental que par la disposition interne des parties » (Lévi-Strauss, 1990). C'est, dans une logique de bricolage savant, un peu comme cela que la créativité se développe au quotidien, même si, par ailleurs, des ruptures scientifiques majeures conduisent à des transformations d'importance (ex. transistor, éclairage électrique, etc.).

Avec l'impression 4D (comme en 1984 avec la 3D), il s'agit, en principe, d'ordonnancer de manière originale des acquis stabilisés associant fabrication additive et matériaux adaptatifs. Léonard de Vinci, au XVIe siècle aurait écrit : «Prenez vos leçons dans la nature, c'est là qu'est notre futur... »! Le bio-mimétisme et la bio-inspiration sont une approche consistant à étudier la nature sous toutes ses formes (Bœuf, 2014) et l'impression 4D peut être considérée comme un domaine qui peut en être issu. L'écaille de la pomme pin est un modèle exemplaire pour associer une démarche de bio-inspiration à l'impression 4D. Il s'agit d'un actionneur hygro-morphe dont la réponse est déclenchée par une variation d'humidité et donc de teneur en eau au sein des tissus (Le Duigou, Beaugrand et Castro, 2017 ; Le Duigou et al, 2019 ; Momeni et Ni, 2018). D’autres idées issues du fonctionnement des pattes du gecko s'en rapprochent (Wang et Lee, 2017) ou bien du déplacement des animaux comme les serpents grâce à plusieurs modes de reptation (Boyer, Mauny et Porez, 2019), etc.

Dans la mesure où les pièces 4D admettent une complexité géométrique et une fonctionnalité inédites, certaines étapes d'assemblage peuvent être supprimées, des gains de place peuvent être atteints dans les objets actifs réalisés, tout en réduisant la complexité de l'amont de la chaîne de valeur puisque les intrants sont essentiellement des poudres, des bobines de fil fusibles ou des fluides. Alors, avec l'impression 4D, avec des options issues du bio-mimétisme, on dispose d'une réelle opportunité d'accomplissement, qui n'en est qu'à ces débuts avec un chiffre d'affaire annuel mondial inférieur à 100 Millions $€$ (à comparer aux 80 Milliards $€ /$ an du seul secteur français de la mécanique). Mais, on n'en est pas encore à la démonstration d'une rupture technologique, plutôt à un enjeu à éclairer de ce domaine récent qui est encore de « découvrir » à quoi il peut réellement 
servir. En réalisant un bilan, on peut commencer à examiner si l'impression 4D est effectivement porteuse de ruptures sociétales.

\subsection{De la matière à l'objet 4D - La place de l'imprimante}

« L’ordre n'est qu'un cas particulier et aléatoire du désordre » (Schiffter, 2019).

«Les modèles embryonnaires sont lacunaires et dysfonctionnels. Ils tendent ainsi à rassurer les acteurs installés, qui continuent de juger leur offre plus pertinente. Or, cela n'a évidemment qu'un temps. Lorsque les modèles alternatifs deviennent plus matures et engagent une dynamique de massification, les opérateurs ont beau saisir le danger réel, il est trop tard » (Zerbid et Mamavi, 2020). Alors, dans ce paragraphe, il a paru important d'essayer de mettre en correspondance promesses scientifiques et exploitations futures par les entreprises du potentiel 4D. Cependant, l'innovation dans ces dernières intervient dans un processus complexe, cumulatif et culturel associé à l'accumulation de compétences (Dosi, 1988 ; Patel et Pavitt, 1997 ; Pavitt, 2005). Elle diffère considérablement d'un secteur à l'autre en termes de degré d'opportunité technologique et de cumul, de bases de connaissances et de barrières technologiques à l'entrée (Marsili, 2000) avec des hétérogénéités considérables (Dachs et Palcic, 2020). Pour qu'il y ait disruption, il faut aller au-delà des promesses et des présentations spectaculaires pour attirer les entreprises dans le changement.

\subsubsection{Une question de définition}

En utilisant une impression 3D, Ge et al (2016) montrent sur la figure 2 comment il est possible de faire évoluer la forme d'un objet 3D en fonction de la stimulation (thermique) qu'il reçoit.

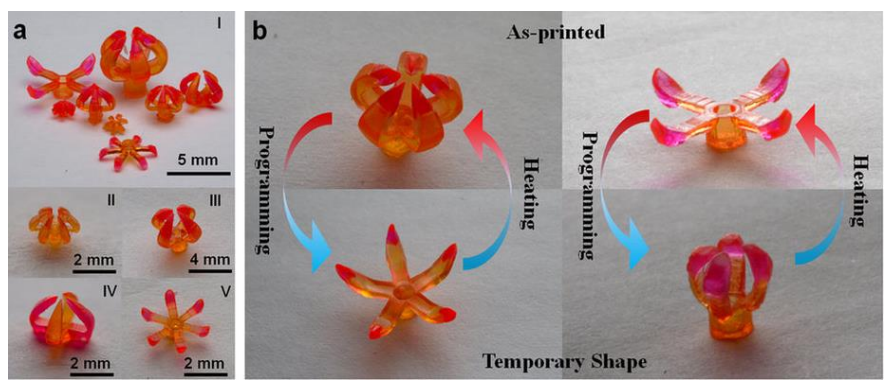

Figure 2. L'anisotropie du matériau crée une courbure lors du changement de la température - Des morphologies complexes (ici une fleur) sont générées avec des évolutions dans les formes des objets

Cette figure résulte d'une utilisation d'un procédé $3 \mathrm{D}$, de matériaux thermosensibles et d'une stimulation. Sur cette base, la figure 3 encadre ce que l'on entend par impression 4D. Elle présente le principe général associé au passage par un procédé classique de fabrication additive : il faut tout d'abord que l'on puisse concevoir et construire un objet et dans une deuxième phase être capable de faire évoluer sa forme à partir d'un stimulus spécifique. Il y a donc dans cette opération couplage matériaux-procédés-fonctionnalité. En dehors des méthodes présentées dans ce document, les technologies de fabrication 3D commencent à se stabiliser (André, 2017) avec leurs avantages et leurs défauts. Pour atteindre un objectif applicatif réel, la mutualisation interdisciplinaire est nécessaire, mais a également ses propres limites. 


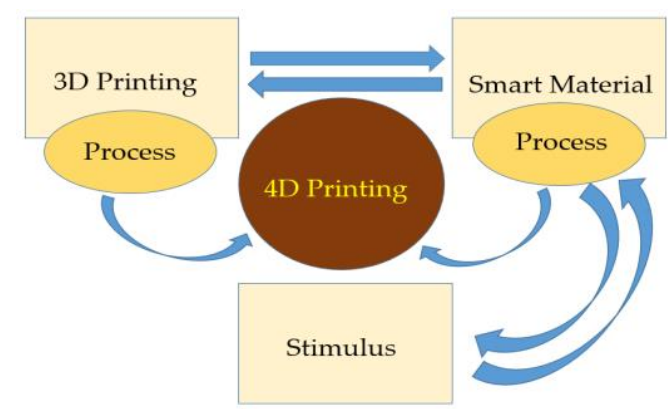

Figure 3. Couplage Procédés -Matériaux en impression 4D

Par définition, l'impression 4D résulte de l'utilisation d'un processus de fabrication additive avec un ou plusieurs matériaux, dont au moins un est stimulable. Elle sera appelée Impression 4D homogène dans la suite de ce document. Avec des matériaux homogènes, il est envisagé de jouer sur la forme pour réaliser des actionneurs intégrés (Zolfagharian et al, 2019 ; Momeni et al, 2017) et l'investir la robotique souple (Zolfagharian, Kaynak et Kouzani, 2020), la fonctionnalité par exemple pour des vêtements ou des changements de couleur pour des applications militaires, etc.

Par exemple, pour compléter le propos, Airbus développerait des fibres de carbone programmables comme économiseurs de carburant et purificateurs d'air ambiant (Rander, 2020). Pour Garcia (2020), la robotique est limitée par les matériaux «durs » utilisés ; la robotique « douce » devrait résoudre ce problème en utilisant certains matériaux souples stimulables. De même, dans l'industrie textile, l'impression 4D permettrait de fabriquer des vêtements qui s'adaptent à la forme et au mouvement du corps. Par exemple, cité par Garcia (2020), l'armée américaine testerait des vêtements qui peuvent modifier leur couleur selon l'environnement ou réguler la transpiration des personnes en fonction de la température extérieure. Le besoin croissant d'objets souples ou flexibles dans diverses applications, telles que les emballages pliants, les éoliennes adaptables, etc., alimente l'émergence de l'impression 4D, l'impression de différents matériaux qui forment une anisotropie matérielle et qui permettent à l'objet de modifier la structure en se pliant, s'allongeant, se tordant et s'ondulant le long de ses axes (Future Bridge, 2020).

Même s'il est encore difficile de savoir quels sont les domaines applicatifs essentiels, plusieurs études donnent des indications sur la répartition des activités. Pour Market Analysis Report (2017), en Chine, les efforts principaux concerneraient les applications militaires, l'espace, l'aéronautique, l'automobile, les textiles «intelligents » et la santé. Pour Market Intellica (2019), quatre quarts approximatifs dessinent le paysage de la 4D : l'espace, l'aéronautique, l'automobile et des divers.

\section{REMARQUE : Bio-printing}

Le bio-printing est une forme d'impression 4D dans laquelle les matériaux sont, au moins en partie, constitués de matière vivante. Ce thème qui a sa propre destinée (André, 2020 ; Danzo et André, 2020) ne sera pas abordé dans cet article.

\subsubsection{Veille active et démarche entreprise}

Il résulte des actions des hommes quelque chose d'autre que ce qu'ils ont projeté et atteint, que ce qu'ils savent et veulent immédiatement. Ils réalisent leurs intérêts, mais il se produit en même temps quelque autre chose qui y est cachée, dont leur conscience ne se rendait pas compte et qui n'entrait pas dans leurs vues » (Hegel, 2003). 
Une veille peut être catégorisée (Peguiron, 2008 ; Raymond, 2000) en fonction des éléments suivants :

- Domaine d'application : veille économique, scientifiques, technologique ;

- Objet d'observation : veille concurrentielle, sectorielle, technologique, client, fournisseur, brevet, produit, image, tendances, etc. ;

- Entreprise qui en a la charge ;

- Moyens utilisés : contacts humains, open source, prospective, web (Angelovska et Mavrikiou, 2013), etc. ;

- Finalité : veille stratégique, tactique ou opérationnelle ;

- Caractéristiques temporelles : veille continue, ponctuelle, anticipative, prospective, conjoncturelle.

La veille créative (Sadok, Benabdallah et Lesca, 2003) se fonde sur l'observation de signaux faibles, mais passés. Elle a pu, depuis 2013 être menée sur ce sujet par les auteurs, compte tenu du nombre modeste (mais en forte augmentation) d'informations issues de la littérature grise et des publications scientifiques publiées. La veille prospective (Antoine, 1992), par son travail d'imagination des opportunités et des menaces possibles pouvant porter à moyen ou long terme sur la légitimité des actions de recherche (Dorow et al, 2015), s'appuyant sur les acquis fait l'objet de la présente publication.

Les réflexions associées à cette mise en situation du thème émergent concernant l'impression 4D permet d'envisager des éventualités qui, par rapport à la prospective, peuvent être considérées comme décalées, voire transgressives. De la sorte, nous avons essayé d'imaginer de nouvelles idées sans contrainte, tout en s'appuyant sur l'existant (Barbieri-Masini, 2000) sans chercher comme Ju et al (2020) à représenter et à modéliser l'incertitude et à structurer l'imagination (Grosul et Feist, 2014). En ce sens, de nouveaux processus d'innovation, en identifiant des verrous et des problèmes non résolus, de besoins en rapport avec des solutions qui leur sont proposés (ou pas), ainsi que des voies d'exploration possibles, seront proposés. Dans notre approche d'utilisation des connaissances scientifiques existantes ou en cours pour l'application, l'innovation a été considérée comme un processus débouchant sur l'intégration couronnée de succès d'une création ou d'une invention sur un marché ou dans une organisation (Alter, 2000).

\subsubsection{Emergence des recherches et bilans économiques}

En comparaison avec la technologie «mère », l'impression 3D (30 milliards $€ /$ an), le marché mondial actuel de l'impression 4D est encore modeste comme l'attestent les données rassemblées dans le tableau 1. Cependant, il est important de noter que pour les auteurs cités dans ce tableau, les taux de croissance sont sensiblement deux fois plus importants que celui de la fabrication additive «traditionnelle». 


\begin{tabular}{|l|l|l|l|}
\hline $\begin{array}{c}\text { Marché mondial } \\
\text { (Millions US\$) }\end{array}$ & \multicolumn{1}{|c|}{$\begin{array}{c}\text { Valeur en Millions US\$ } \\
\text { (Année) }\end{array}$} & \multicolumn{1}{|c|}{$\begin{array}{c}\text { Taux d'augmentation } \\
\text { annuel (\%) }\end{array}$} & \multicolumn{1}{c|}{ Référence } \\
\hline $62(2019)$ & $488(2025)$ & 42 & Mordor Intelligence (2020) \\
\hline Environ 60 (2019) & $540(2025)$ & 43 & Market and Market (2017) \\
\hline $65(2019)$ & Environ 450 (2025) & Environ 40 & Market Analysis Report (2019) \\
\hline $52(2018)$ & $440(2026)$ & 31 & VMR (2019) \\
\hline & $420(2026)$ & 26 & PMR (2020) \\
\hline & $250(2028)$ & 28 & NKWR (2019) \\
\hline & $240(2028)$ & & Market Screener (2019) \\
\hline $12(2015)$ & $420(2026)$ & 33 & Market Watch (2020) \\
\hline & & 40 & VSR (2019) \\
\hline $35(2019)$ & $200(2025)$ ou 100 2025) & 33 ou 20 & Research and Market (2016) \\
\hline
\end{tabular}

Tableau 1. Estimation des marches de l'impression 4D et son évolution temporelle

A l'évidence, en dehors d'un intérêt lié à quelques applications encore très modestes en termes économiques, citées plus haut, on est très loin d'un succès effectif; mais avec de tels taux de croissance de $40 \%$ comme ceux estimés par des officines citées en référence, on pourrait arriver à 12 milliards €/an dans 10 ans ! Il s'agit d'un marché supérieur à celui qu'avait en 2000 la fabrication additive ! Le regard sur le sujet est donc fondé.

D'un point de vue scientifique, on dispose de données par les publications évaluées par les pairs issues du site du CNRS. Les résultats quantitatifs bruts font l'objet de la figure 4 avec, comme seul mot clé utilisé, «4D printing ». Il y a une corrélation des données avec une évolution exponentielle avec un taux de doublement impressionnant de l'ordre de 17 mois !

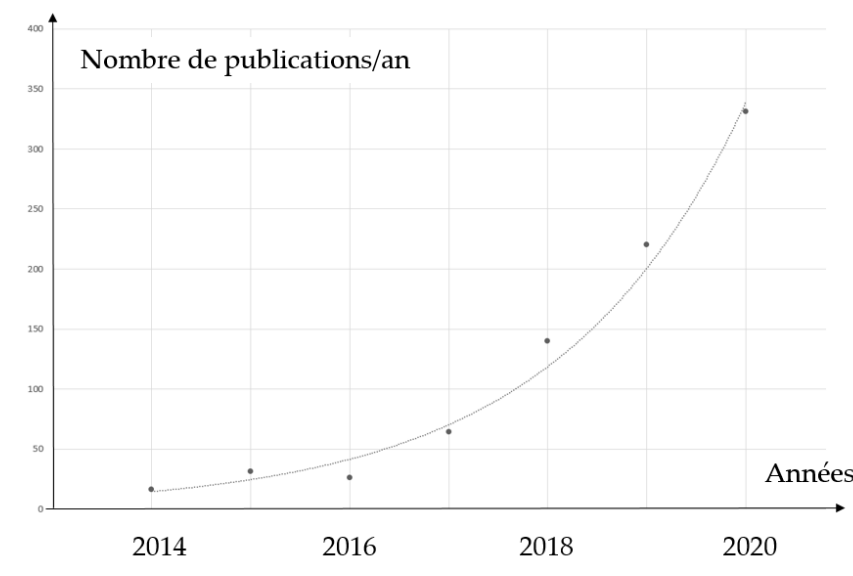

Figure 4. Evolution exponentielle du nombre de publications annuelles sur le thème 4D Printing

\subsection{Contraintes et limites de l'impression 4D}

«A moins que que je ne veuille allumer une lanterne pour faire voir le soleil » (More, 1987). 
Les auteurs s'accordent sur l'association rappelée sur le figure 2 entre une méthode d'impression 3D, de matériaux adaptatifs et d'une stimulation pour agir sur la forme et/ou la fonctionnalité des objets créés. Comme on va le montrer ci-après, chaque contributeur au domaine apporte ses propres contraintes conduisant en final à la réalisation de dispositfs dont l'opérationnalité est questionnée. Il s'agit ici d'aider à comprendre la réalité des questions posées et des réponses de la science pour permettre ultérieurement de centrer les débats de fond sur la hiérarchisation des arguments en présence et, autant que faire se peut, les choix les plus pertinents pour valoriser la technologie. L'aspect scientifique ou technique des observations relève des sciences, de épistémologie et de la conduite des projets, mais également des sciences humaines et sociales, notamment l'économie et la sociologie.

\subsubsection{Matériaux classiques utilisés en impression 4D homogène}

«L'exigence d'un savoir en transformation, amélioration et croissances continues, n'a rien d'utopique » (Chalmers, 1990).

Les matériaux doivent disposer d'un module d'élasticité faible ou décroissant pour participer à la déformation. Ils font partie des familles suivantes : polymères, colloïdes, mousses, émulsions et matière granulaire. Plusieurs modes de stimulation peuvent être évoqués permettant de produire un objet 3D par fabrication additive et des modifications de forme induite par une stimulation (cf. par exemple, Ryan, Down et Banks, 2020).

\section{REMARQUE : fonctionnalité}

S'il existe de nombreux travaux sur les changements de fonctionnalité, en particulier sur les changements de couleur, ceux-ci pour des raisons de linéarité des réflexions ne sont pas considérés ici (cf. cependant, Piedrahita-Bello et al, 2020). Toutefois, une grande partie des commentaires présentés dans cet article peuvent leur être appliqués.

\subsubsection{Polymères actifs - Muscles photochimiques}

Une approche concerne l'effet de la lumière sur une structure polymérique (Nakano, 2010 ; Yoshino et al, 2010 ; Yu et al, 2003 ; Kuksenok et Balzs, 2013 ; Khim et al, 2014 ; Yu et al, 2015 ; Khoo et al, 2015; Fang et al, 2017 ; Jin et al, 2018) : 1'irradiation induit des rotations internes dans le matériau, ce qui contribue à changer la géométrie de la zone irradiée conduisant à des déformations qui dépendent du flux reçu; celles-ci peuvent être réversibles si l'on dispose de matériaux photochromes (transformation provisoire, réversible). L'ensemble des processus en dehors de la photo-dégradation est représenté sur la figure 5.

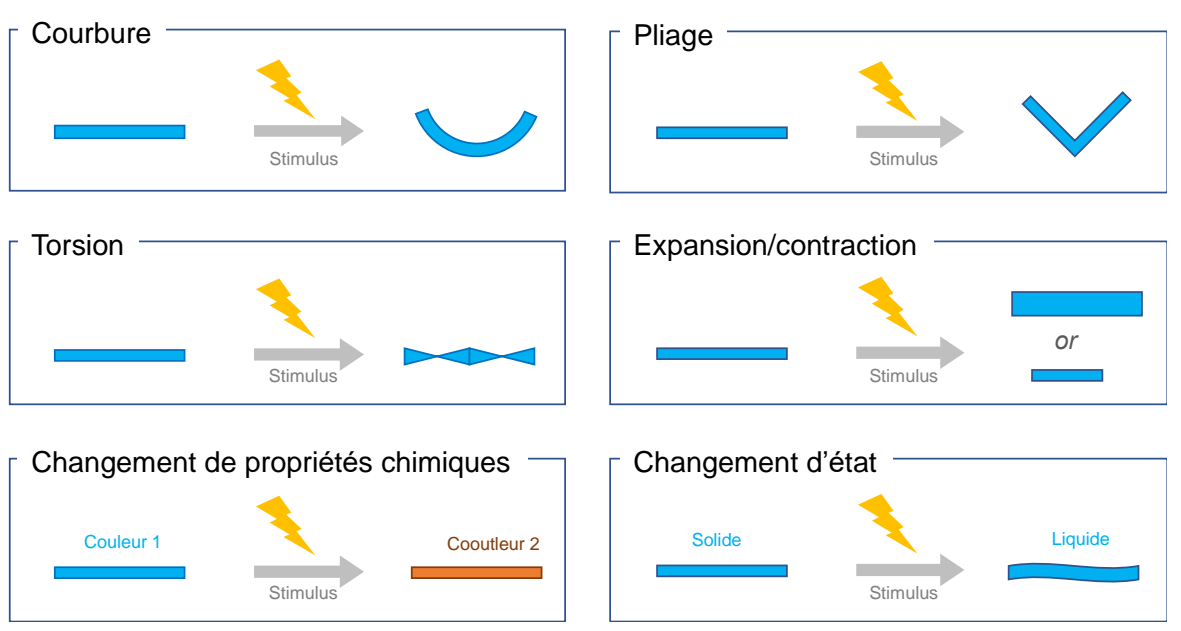

Figure 5. Mécanique de déformation de polymères sous stimulation 
Parmi les exemples, la déformation importante de polymères contenant des groupes azo-benzène (cf. Mahimwalla et al, 2012) est à noter (cf. figure 6). Ces groupements, par irradiation passent de configurations Trans à Cis selon la longueur d'onde utilisée (voir également De Simone, Gidoni et Novelli, 2015 ; Rosales et al, 2015 ; Liu et al, 2017 ; Boydston et al, 2018 ; Zhang et al, 2019).
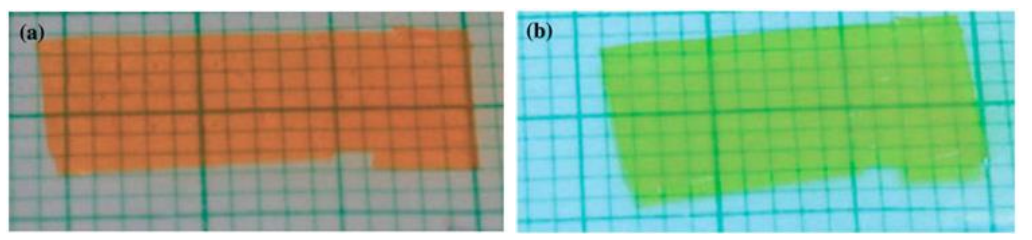

Figure 6. Effet de l'irradiation sur un film de polymère contenant des groupes azo-benzène : (a) avant irradiation ; (b) aussitôt après selon Mahimwalla et al (2013)

La figure 7 aimablement fournie par Qi Ge (extraite de Ge et al, 2016) illustre une transformation réversible d'un polymère qui se déforme avec la température. D'autres travaux peuvent répondre à ce principe de transformation (Ercole, Davis et Evans, 2010 ; Cheng et al, 2010 ; Ilic-Slovanovic et al, 2011 ; Petr et al, 2015 ; Zhang et al, 2015 ; Gao et al, 2016 ; Patel et al, 2017).
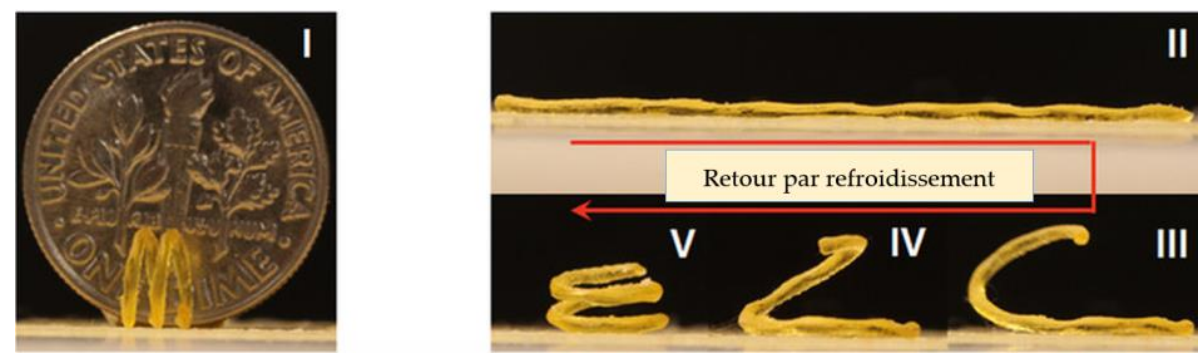

Figure 7. Déformations d'un film de polymère induites par la lumière

\subsubsection{Polymères actifs - Effets thermiques sur des polymères}

Pour Zhao, Qi et Xie (2015), il est possible de disposer de matériaux polymériques dont la forme peut dépendre soit de la température, soit de transformations photochimiques. Il s'agit pour ces auteurs de trouver des réactions réversibles affectant l'espace entre molécules ou macromolécules (cf. figure 8) (voir également Zare et al, 2019 ; Xin et al, 2019 ; Goo, Hong et Park, 2020).

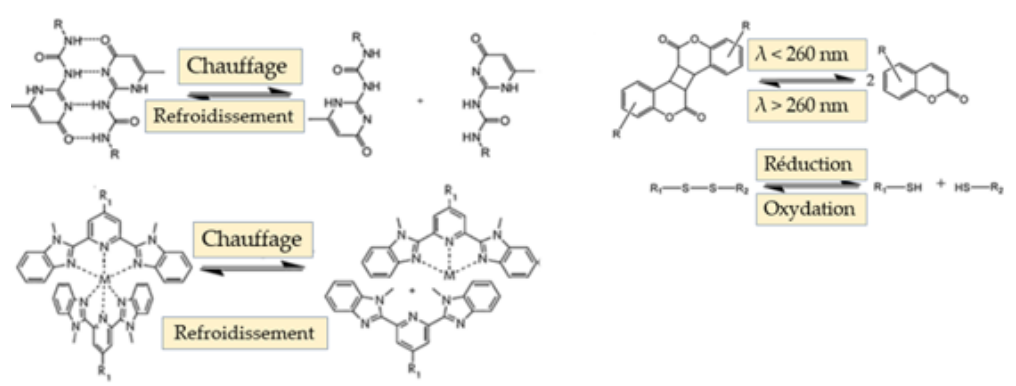

Figure 8. Systèmes réversibles avec changement de volume

Un exemple explicatif est donné sur la figure 9 illustrant le changement de forme (voir également Zhao, Qi et Xie (2015) sur l'analyse et la description de différents matériaux actifs). 


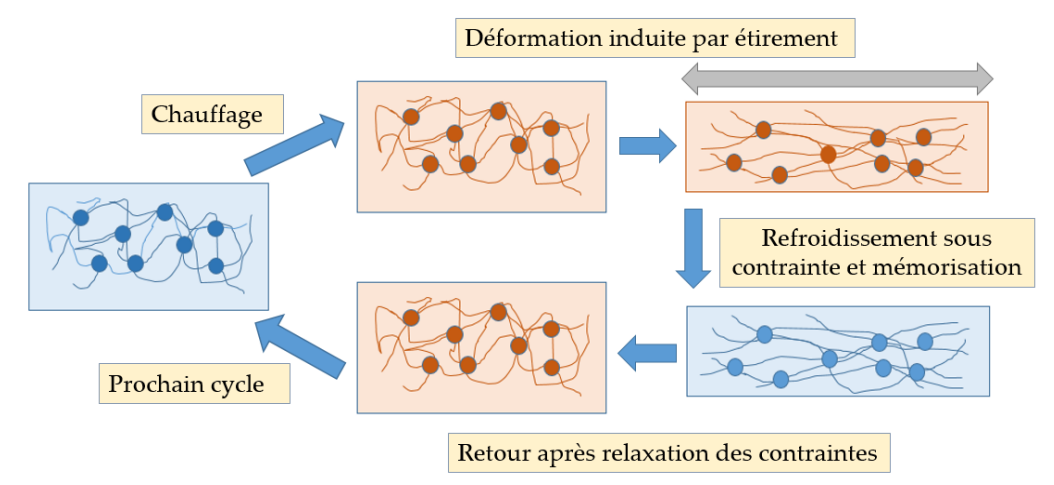

Figure 9. Principe des déformations réversibles induites thermiquement

Des encres réalisées à base d'hydrogels sont capables de déformations induites par l'humidité et par la température (André, 2017). Le Duigou et al (2016) ont utilisé des particules de bois comme charge dans un polymère. Orientées dans le cisaillement d'extrusion de ce polymère fusible, elles gardent la mémoire de leur orientation en dessous du point de gel du matériau et peuvent voir leur forme évoluer en présence d'humidité (ou d'un chauffage). Enfin, d'un point de vue optimisation Zhang et al (2011) ont ajouté au matériau sensible (hydrogel) à la chaleur et à la lumière, le poly-(Nisopropylacrylamide) (pNIPAM), des charges en nanotubes de carbone qui facilitent le transfert de chaleur dans la masse du matériau et l'absorption de lumière en surface. Han et al (2012) ont exploité des transformations induites par le $\mathrm{pH}$, etc.

\subsubsection{Polymères actifs - Effets de gonflement dans des polymères}

D'autres phénomènes physiques peuvent être exploités pour voir changer de forme un objet 3D réalisé dans un matériau sensible à un environnement: $\mathrm{pH}$, présence d'un solvant, etc. (Bakar et Djaider, 2007 ; Liu et al, 2017, Shiblee et al, 2018). Le gonflement des réseaux de polymères réticulés en présence d'un solvant est un phénomène bien connu (cf. par exemple Ancla, 2010 ; Bounouira, 2015). Dans ce dernier cas, présenté figure 10, on peut observer des modifications très sensibles de la forme d'un objet 3D.

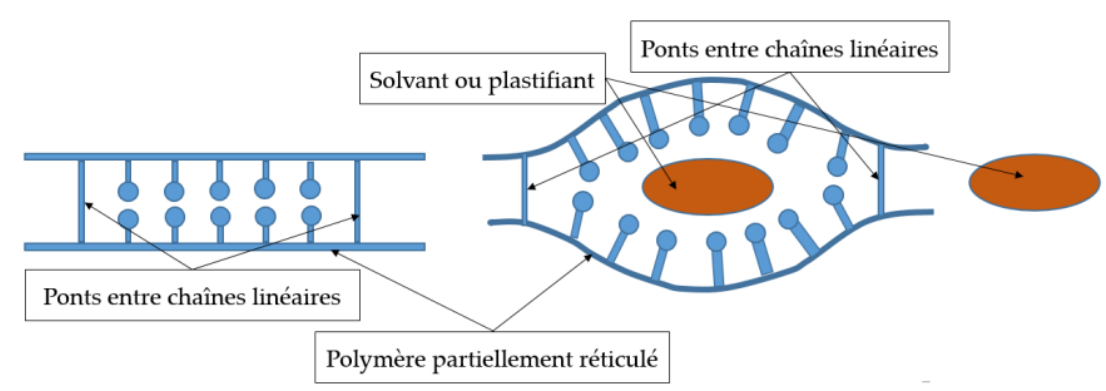

Figure 10. Changements de forme en présence d'un solvant

Avec certains solvants, les tronçons de chaîne entre deux points de réticulation se déplient comme dans le cas des chaînes linéaires, mais ce mouvement est limité par l'existence des ponts. Le phénomène se limite donc à un gonflement, d'autant plus prononcé que le taux de réticulation est faible.

\section{REMARQUE : hydrogel}

Le terme d'hydrogel définit des matériaux dotés d'une composante liquide et d'une composante solide. Ils sont composés de chaines de polymères assemblés via le processus de réticulation et peuvent contiennir des liquides comme de l'eau. Les hydrogels peuvent être utilisés en fabrication additive et voir leur forme évoluer en présence d'un solvant. 
Ces quelques lignes illustrent une assez grande richesse de matériaux essentiellement organiques sensibles à un environnement : d'un stimulus appliqué peut résulter un changement de forme.

\subsubsection{Polymères électro-actifs}

Les polymères électro-actifs, ou EAP, sont des polymères qui présentent un changement de taille ou de forme lorsqu'ils sont stimulés par un champ électrique. Les applications les plus courantes de ce type de matériaux sont les actionneurs et les capteurs. Une propriété caractéristique typique d'un EAP est qu'il subira une grande quantité de déformations tout en maintenant des forces importantes (Bar-Cohen, 2004 ; Lendlein, 2010 ; Hu et al, 2012 ; Hager et al, 2015 ; Lendlein et Gould, 2019 ; Duduta et al, 2019; Hisour, 2020)). A la fin des années 90, il a été démontré que certains EAP pouvaient présenter une réduction de taille considérable allant jusqu'à $380 \%$ de la taille initiale, ce qui est beaucoup plus que tout actionneur en céramique. L'une des applications les plus courantes des EAP est la robotique souple dans le développement de muscles artificiels (Maziz et al, 2017). L'EAP possédant une caractéristique de transformation réversible, peut avoir plusieurs configurations, mais est généralement divisé en deux classes principales: diélectrique (Du et al, 2015) et ionique. Une difficulté est hélas à noter; si les caractéristiques de ces matériaux sont remarquables, il n'existe pas à notre connaissance de fabrication de ces polymères par fabrication additive.

\subsubsection{Entre objet 3D et stimulations}

«Dans la littérature, [le créatif] est souvent vu comme un observateur curieux, facilement distrait, intuitif, judicieux, maniant l'humour, alerte, présomptueux, mais il est aussi difficilement gérable, car il a un esprit indépendant et un comportement imprévisible » (Bourcier et Van Andel, 2017).

Avec des d'objets 3D contenant massivement de la matière qui peuvent être activés par différents stimuli ( $\mathrm{pH}$, chaleur, lumière, champ électromagnétique, etc. - cf. André, 2020 a), il faut réaliser une pièce en fabrication additive avec des matériaux actifs, suivie d'une stimulation localisée dans l'espace et dans le temps pour faire évoluer la forme 3D d'un objet. Plusieurs méthodes (cf. figure 11) peuvent être retenues en termes de mouvements réversibles avec différents stimuli externes à l'objet 3D (cf. par exemple Ge et Dunn, 2013 ; Sossou et al, 2019 ; 2019 a ; André, 2017) :

- Polymères sensibles à l'humidité, à la chaleur : soit le polymère absorbe de l'eau, soit il en perd, sa densité change avec un éventuel rétrécissement (gonflement/dégonflement). Il existe également des systèmes réversibles sensibles à la chaleur (polymères à mémoire de forme) avec des changements de densité ;

- Les «muscles » photochimiques : l'irradiation induit des rotations internes dans le matériau (exemple classique de l'azo-benzène et de ses dérivés), ce qui contribue à modifier la géométrie de la zone irradiée, entraînant des déformations qui dépendent du flux lumineux reçu ; celles-ci peuvent être réversibles si des matériaux photochromiques sont disponibles (transformation réversible; bistabilité).. ;

- Avec les systèmes multi-matériaux, il est possible de jouer sur la différence de comportement des matériaux avec une stimulation pour produire des effets de type « bimétal » ou bilame ;

- Avec des matériaux chargés, un champ électromagnétique peut induire une orientation de la charge conduisant à une déformation souhaitée de l'objet 3D ;

- Etc. 


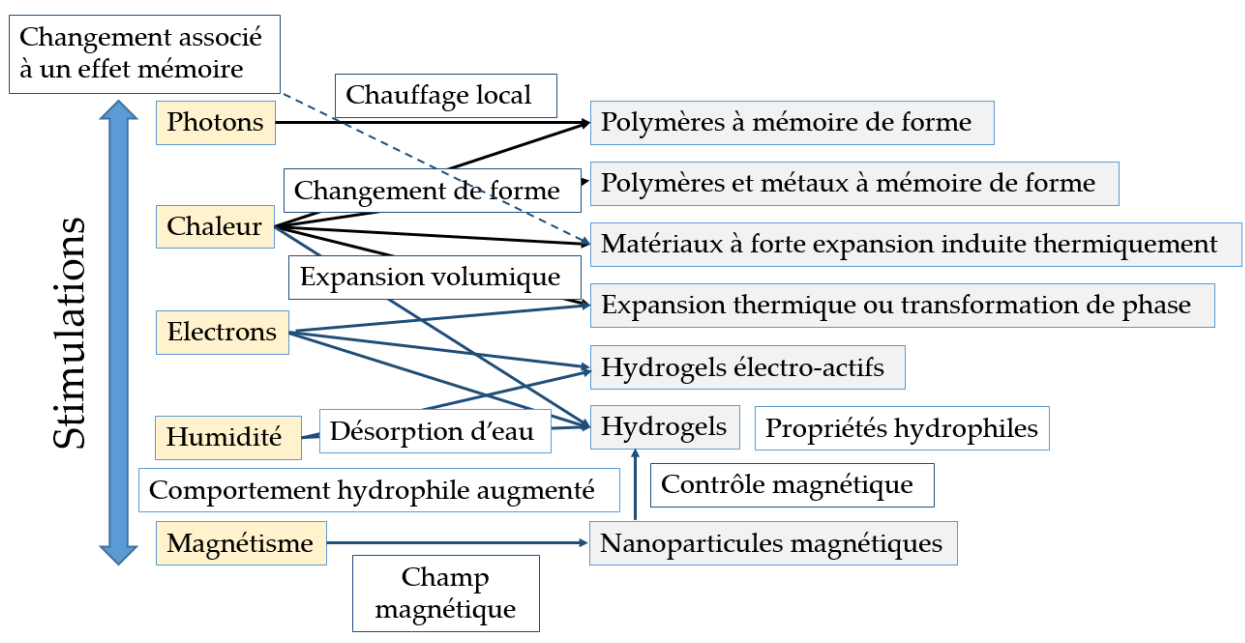

Figure 11. Les différents modes de stimulation selon Ahmed, Musbah et Atiyah (2020) et Zhang, Demir et Gu (2019)

Le tableau 2 issu des considérations présentées dans André (2020 a) et dans Momeni et Ni (2020) rappelle les intérêts et les limitations des procédés 4D massifs homogènes. La connaissance de ces résultats synthétiques présentés dans ce tableau servira ensuite à prendre en considération des aspects prospectifs au $\S 3$ concernant les développements scientifiques et technologiques de l'impression 4D.

\begin{tabular}{|l|l|}
\hline Impression 4D & Commentaires (cf. André, 2020 a) \\
\hline Attractivité & $\begin{array}{l}\text { - Procédé 3D permettant en une seule étape de fabriquer un objet et de disposer ensuite de } \\
\text { modes d'actuation plus ou moins homogènes à l'intérieur de l'objet massif } \\
\text { - Utilisation de modes de stimulation externes pour faire évoluer la forme (cf. figure 5) et/ou } \\
\text { la fonctionnalité de l'objet } \\
\text { - Grand nombre de modes de stimulation (mécanique, chauffage, humidité, pH, lumière et } \\
\text { autres champs électromagnétiques) } \\
\text { - Champ d'application envisagé très vaste (en termes de formes ou de fonctionnalités) } \\
\text { - Aspect spectaculaire évident }\end{array}$ \\
\hline $\begin{array}{l}\text { Limitations } \\
\text { - Choix limité de matériaux actifs, à très forts coefficients de déformation, essentiellement } \\
\text { par conséquence, à propriétés mécaniques modestes }\end{array}$ \\
$\begin{array}{l}\text { - Difficulté de localiser la stimulation sur l'objet } \\
\text { - Anisotropie de la stimulation } \\
\text { - Taille du dispositif de stimulation relativement à celle de l'objet 4D } \\
\text { - Temps de réponse long suite à une stimulation, incompatible avec des usages industriels } \\
\text { (plusieurs minutes) } \\
\text { - Energie de la stimulation susceptible d'endommager l'objet } \\
\text { - Anisotropies liées au mode de fabrication par additivité } \\
\text { - Tenue dans le temps } \\
\text { - Modélisation des déformations volumiques difficile (Sossou et al, 2019) }\end{array}$ \\
\hline
\end{tabular}

Tableau 2. Attractivités et limitations actuelles de l'impression 4D

Soit on se contente d'un environnement global qui évolue (température, humidité, etc.) et auquel cas, il n'y a pas de dispositif à implanter, soit on souhaite faire évoluer localement la forme de l'objet. Dans ce cas, le dispositif de stimulation doit impérativement être en mesure d'intervenir dans des zones précises de l'espace. Ce que l'on montre dans André (2020), c'est qu'il est peu réaliste d'utiliser des modes de chauffage localisés (transfert de chaleur dans l'objet) ou d'excitation lumineuse (absorption non aisément localisée sur les zones actives, chauffage lié à des transitions non radiatives, effets d'ombre, etc.). Ce que l'on montre donc, c'est qu'il est difficilement envisageable d'utiliser des modes de stimulation tels qu'envisagés dans les publications pour 
réaliser des dispositifs d'utilisation industrielle courante. Cette considération introduit bien le paragraphe suivant sur d'autres limites.

\subsection{Limites et contraintes}

« C'est donc une chose toute avérée, que l'origine des plus grands et des plus durables sociétés, ne vient point d'une réciproque bienveillance que les hommes se portent, mais d'une crainte mutuelle qu'ils ont les uns des autres » (Hobbes, 1993).

Les entreprises devraient faire preuve d'audace en matière d'innovation, quitte parfois à essuyer quelques échecs ne mettant pas en cause leur survie. Ce qui a été montré dans André (2017) pour l'impression 3D, c'est que le processus conduisant au développement et à l'introduction d'une technologie perturbante peut être difficile pour différentes (bonnes) raisons, alors qu'elles doivent, selon les décisions passées de l'entreprise, ses activités habituelles, ses ressources accumulées et sa culture, «se moderniser tout en restant ancrées dans la tradition » (Celhay et Cusin, 2011). Entre l'introduction d'un procédé valide et sa mise en œuvre industrielle, il faut du temps. Pour l'impression 3D, il aura fallu plus de 20 ans environ. Il n'y a aucune raison que cette durée soit très différente pour la 4D, introduite en 2013, pour autant qu'elle dispose des mêmes types d'atouts que celle qui a été à son origine. Il est donc important de rappeler, outre les nombreux avantages de la fabrication additive que l'on retrouve dans de nombreux ouvrages et publications (cf. Berchon, 2020 ; Weller, Kleer et Piller, 2015 ; Frigant, 2020 ; Rafiee et al, 2020), que l'impression 3D qui est encore en développement ( $20 \%$ environ de croissance annuelle des nombres de publications.) Elle reste une technologie attractive, mais encore fragile.

La figure 12 issue en partie de Kanu et al (2019) met en évidence l'ensemble des sous-thèmes à maîtriser pour une impression 4D optimisée.

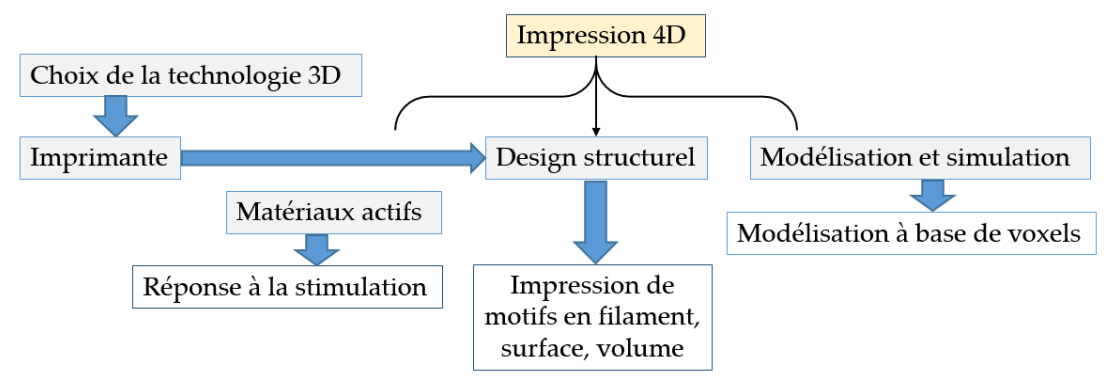

Figure 12. Sous-thèmes concernés en impression $4 D$ à maîtriser

\subsubsection{De l'impression 3D à la 4D}

«Une forme d'activité subsiste parmi nous qui, sur le plan technique, permet assez bien de concevoir ce que, sur le plan de la spéculation, peut être une science que nous préférons appeler « première » plutôt que primitive : c'est celle communément désignée par le terme de bricolage » (Lévi-Strauss, 1990).

«Par essence, la fabrication additive génère de l'inséparabilité technologique dès lorsqu'il s'agit de produire une pièce au sein de l'imprimante, plutôt que par activités organisées en différents stades successifs » (Frigant, 2020). Comme le domaine 4D exploré ici prend sa source et son devenir par l'usage de la fabrication additive, il nous a paru utile de rassembler quelques éléments associés à ce domaine relativement stabilisé dans le tableau 3 (avec des ouvertures sur l'impression 4D). 


\begin{tabular}{|c|c|c|}
\hline Impression 3D & Impression 4D & Commentaires \\
\hline $\begin{array}{l}\text { Cadre général (André, } \\
\text { 2017) }\end{array}$ & $\begin{array}{l}\text { Cadre général (Pei et Loh, } \\
\text { 2018) }\end{array}$ & 3 devenant 4 degrés de liberté... \\
\hline Personnalisation & Personnalisation & Frigant, 2020 \\
\hline $\begin{array}{l}\text { Utilisation en conditions } \\
\text { extrêmes (ex. spatial) }\end{array}$ & $\begin{array}{l}\text { Utilisation en conditions } \\
\text { extrêmes (ex. spatial) }\end{array}$ & Chen et al, 2019 ; Rander, 2020 ; Garcia, 2020 \\
\hline $\begin{array}{l}\text { Simplification des } \\
\text { assemblages }\end{array}$ & Simplification des assemblages & $\begin{array}{l}\text { Cf. robotique souple (Rafsanjani, Bertoldi et } \\
\text { Studart, } 2020 \text {; Jiang et al, 2020) }\end{array}$ \\
\hline $\begin{array}{l}\text { Débats sur les aspects } \\
\text { financiers et écologiques }\end{array}$ & $\begin{array}{l}\text { Débats sur les aspects } \\
\text { financiers et écologiques }\end{array}$ & Cf. André, 2020 \\
\hline $\begin{array}{l}\text { Inhomogénéité du } \\
\text { processus de fabrication }\end{array}$ & Inhomogénéité du processus & $\begin{array}{l}\text { Notion de fabrication additive avec des } \\
\text { anisotropies (André, 2017) }\end{array}$ \\
\hline Conception numérique & $\begin{array}{l}\text { Modélisation et conception } \\
\text { plus complexes des } \\
\text { déformations et donc de la } \\
\text { conception }\end{array}$ & Kanu et al, 2019 \\
\hline Cyber-sécurité et copies & Cyber-sécurité et copies & Chan et al, 2018 ; Schwab, 2017 \\
\hline Voxels identiques & Voxels identiques & Temps perdu dans la fabrication \\
\hline $\begin{array}{l}\text { Structures fermées } \\
\text { impossibles à réaliser }\end{array}$ & $\begin{array}{l}\text { Structures fermées impossibles } \\
\text { à réaliser }\end{array}$ & $\begin{array}{l}\text { Besoin de fabrication 3D volumique (cf. Zyla et } \\
\text { al, } 2020 \text {; Stevenson, 2020) }\end{array}$ \\
\hline $\begin{array}{l}\text { Peu de machines « multi- } \\
\text { matériaux» }\end{array}$ & $\begin{array}{l}\text { Peu de machines « multi- } \\
\text { matériaux» }\end{array}$ & $\begin{array}{l}\text { Situation qui peut être critique en } 4 \mathrm{D} \text { pour } \\
\text { réaliser des bilames ou des systèmes complexes } \\
\text { (cf. figure 13). Des progrès existent (Hirsch, } \\
\text { Charlet et Amstad, 2020) }\end{array}$ \\
\hline $\begin{array}{l}\text { Pas d'orientation des } \\
\text { voxels (sauf FDM) }\end{array}$ & $\begin{array}{l}\text { Pas d'orientation des voxels } \\
\text { (sauf FDM) }\end{array}$ & $\begin{array}{l}\text { Difficulté en } 4 \mathrm{D} \text { d'exploiter la position spatiale } \\
\text { des voxels }\end{array}$ \\
\hline
\end{tabular}

Tableau 3. Quelques éléments critiques associés à la fabrication 3D pour la 4D

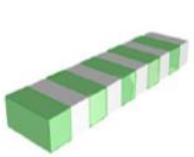

1

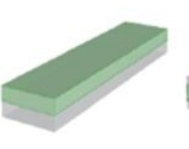

2

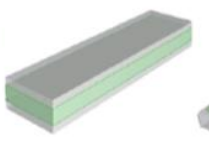

3

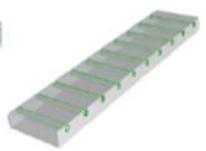

4

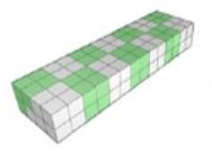

5

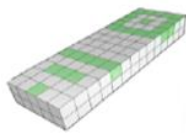

6

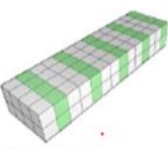

7

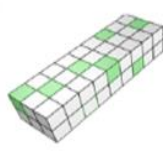

8

(A) 1 : Structure segmentée ; 2 : Bilame ; 3 : Sandwich ; 4 : Renforcée fibres ; 5 : Régulière ; 6 : Gradient ; 7 : spécifique ; 8 : Aléatoire

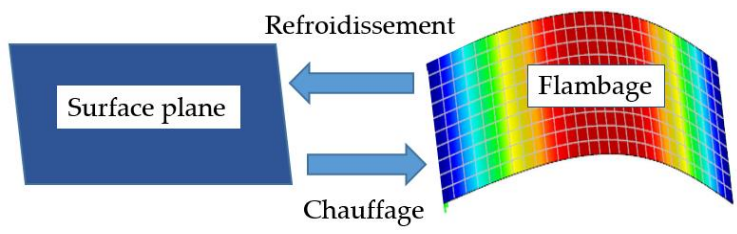

(B)

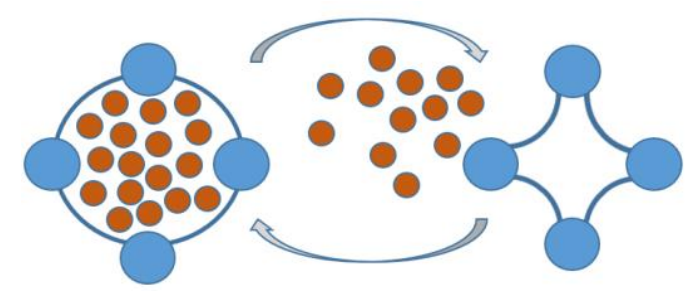

(C)

Figure 13. Intérêts d'une fabrication multi-matériaux pour l'impression $4 D(A)$ : Approche numérique (cf. Jian, 2020) - (B) : Déformations d'une structure 2D (cf. par exemple Tibbits, 2014) - (C) approche continue (cf. Evans et al, 2020) 
Comme le montre cette figure, la présence de matériaux à comportements différents pour la même stimulation peut entrainer des déformations spatiales répondant aux critères de la définition de l'impression 4D. Le développement de machines 3D multi-matériaux dédiées est donc important, voire essentiel, pour celui de la 4D. La modélisation et la simulation avec des voxels pour une distribution donnée lorsque les matériaux actifs sont exposés à un stimulus reste complexe et devra être engagée si l'on veut rentrer dans des solutions à des problèmes inverses. Pour Kanu et al (2019), il est important de modifier l'approche actuelle de modélisation et de simulation basée sur les voxels (qui n'en est qu'à ses tout débuts) pour réaliser de manière efficace des objets $4 \mathrm{D}$, en particulier bio-inspirés (cf. la démarche de Teoh et al, 2017 ; Sossou et al, 2018 ; 2019). La figure 14 , issue de cette dernière référence illustre ce que peut apporter une modélisation sur une poutre.

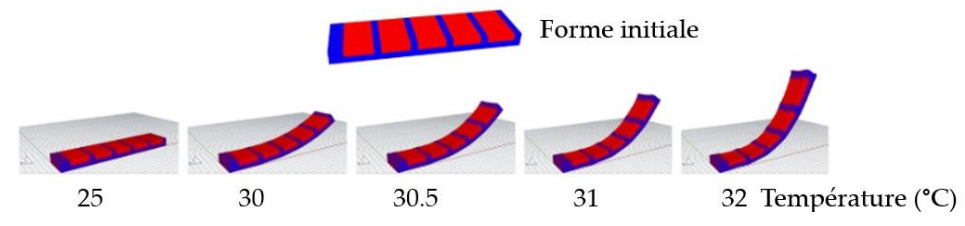

Figure 14. Déformation d'un actionneur à base d'hydrogel

L'impression 4D peut en fait être abordée dans la conception sous différents points de vue, tels que la conception du système, la conception du produit/de la pièce et la conception des matériaux. Dans ce document, le domaine de la connaissance sera limité à la conception de produit/pièce, bien que chaque échelle de conception mérite d'être étudiée. En fait, les artefacts introduits par les questions d'impression $4 \mathrm{D}$ peuvent être considérés comme un réseau de contraintes interdépendantes. L'ontologie envisagée (appelée HERMES) développée par Dimassi et al (2020) permettra une aide à la décision multi-vues et une conception assistée par la connaissance en ce qui concerne la conception pour l'impression 4D. À titre d'exemple, les objectifs d'utilisation potentiels peuvent être répartis entre la planification de la séquence de transformation, la sélection intelligente des matériaux, la distribution intelligente des matériaux, la planification des processus de gestion de l'environnement, pour n'en citer que quelques-uns, et nécessiteront des interfaces de programmation d'applications spécifiques liées aux acteurs concernés. En conséquence, ce cadre (illustré à la figure 15) place l'ontologie HERMES comme un silo sémantique central et hermétique où de multiples raisonnements et requêtes basés sur des règles peuvent être effectués par de multiples acteurs (c'està-dire l'architecte du produit, le concepteur du produit, l'expert en matériaux, le planificateur de processus) ayant des préoccupations diverses dans le processus de conception. Mais, on l'aura compris, il ne s'agit que d'une étape.

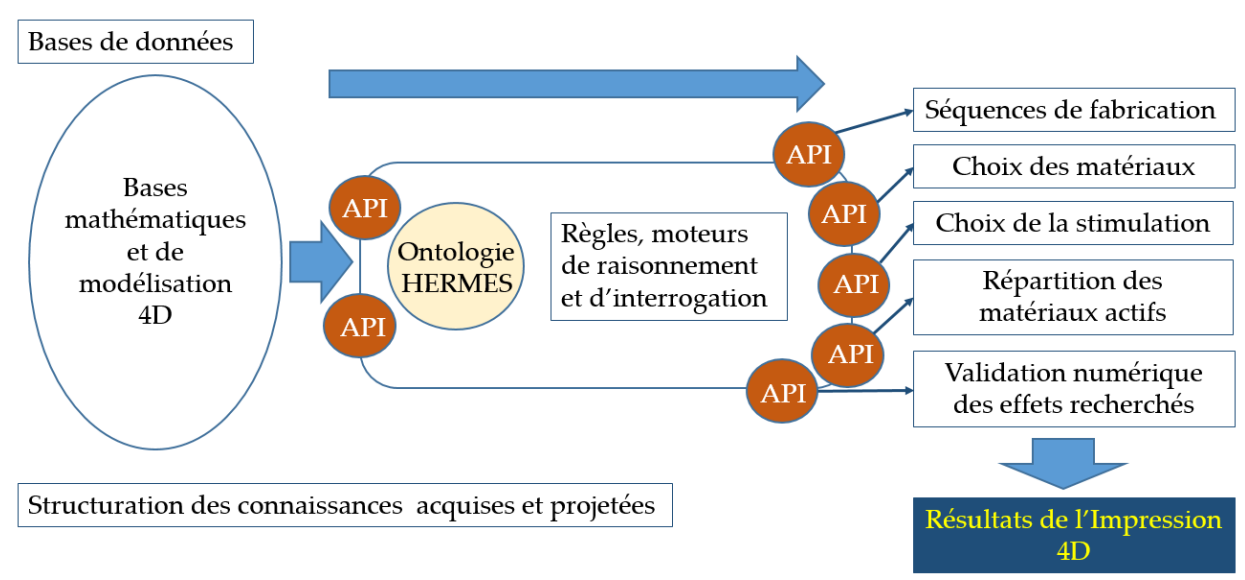

Figure 15. Cadre de construction et d'utilisation s'appuyant sur l'ontologie HERMES (API pour "Application Programming Interface » ou en français «Interface deProgrammation d'Application ») 


\section{REMARQUE : Re-design}

En fabrication additive, en profitant du potentiel de la technologie, il est possible de reconcevoir des formes d'objets pour se rapprocher des fonctionnalités souhaitées (tenue mécanique, transferts de matière et de chaleur améliorés, gains en poids, etc.) (Exemples, Fillingim et al, 2020 ; 4D_Additive, 2020). Ce type d'opération engagé depuis plusieurs années n'a pas encore atteint sa phase de routine. Gageons qu'elle pourra être utilisée comme base de travail pour des logiciels de relation forme - dépôt localisé de matériaux différents fonctionnalités souhaitées-stimulation. Sinon, on laissera une large part de la destinée 4D à l'improvisation, ce qui ne relève pas trop du vocabulaire industriel sur ce point !

Pour revenir à la pomme de pin (Le Digou et Castro, 2020), deux phénomènes sont à l'œuvre : l'orientation des fibres et une structure de bicouche définissant une architecture particulière d'une part et le transfert de l'humidité dans le matériau d'autre part sont responsables des effets d'ouverture des lamelles de la pomme de pin. Avec des machines 3D actuelles, seule une partie de ce besoin est couverte.

Par ailleurs, nous avons rappelé les faiblesses liées aux matériaux actifs qui doivent être suffisamment élastiques pour qu'ils puissent se déformer, avec des modules d'Young modestes (André, 2020) et des difficultés d'implantation et/ou de problèmes physiques liés à la stimulation. Et pourtant, comme on le montre ci-après, il existe une remise en cause modeste de ces difficultés dans les publications sur le sujet.

\subsubsection{Bilan bibliographique}

«L'herbe ne pousse jamais sur la route où tout le monde passe » (Proverbe africain).

«Une discipline scientifique se caractérise ainsi par une autolimitation volontaire par rapport au projet philosophique et donc par une efficacité heuristique et une légitimité épistémologique autre » (Wissmann, 2010).

Une fois installés une discipline, une méthodologie, un procédé, etc., se constituent des groupes d'acteurs ayant des visions communes (sociétés savantes, réseaux d'acteurs, etc.). D'un point de vue historique, l'exemple de Thomas Edison peut être rappelé, imposant un point de vue sur l'utilisation du courant continu (Corazza, 2015). Cette situation qui a pu répondre à des besoins sociétaux a été mise à mal difficilement par Tesla, imposant l'usage du courant alternatif. Mais il a fallu aller audelà de corporatismes, d'habitudes et d'absence de volonté de prendre des risques, ce qui correspond toujours à une démarche difficile surtout si l'on ne dispose pas de motivations profondes ou de solutions alternatives crédibles...

$\mathrm{Au}$ stade de l'idée, presque rien n'a été engagé et tout est encore possible en termes de développements. Pour aller un peu plus loin, il va falloir modéliser et/ou réaliser des preuves de concepts qui ont généralement l'avantage de faire émerger des questions scientifiques, techniques, épistémologiques, etc. Une dynamique inhérente à la recherche (interdisciplinaire ou pas) consiste à se laisser porter par sa curiosité ou à s'engager sur des demandes spontanées ou stimulées vers des questions nouvelles ou des objets nouveaux, cela d'autant plus qu'en science, l'innovation devrait être un critère majeur de réussite. Avec les modes de gestion de la recherche académique, les conservatismes l'emportent souvent sur le créatif et la prise de risque...

Les processus d'innovation incrémentale conduisent cependant parfois vers des objets ou des questionnements qui dépassent ceux définis par les structures cognitives et institutionnelles qui organisent la recherche, c'est-à-dire les disciplines. Alors, l'innovateur qui est parti d'une idée, qui l'a explorée et approfondie peut s'appuyer sur un certain nombre d'hypothèses, sur les éléments de 
sa perception de la réalité avec une projection vers son possible usage. C'est à partir de cette étape qu'il pourra peut-être réaliser une preuve de concept, ce qui peut lui permettre de proposer une ébauche d'utilisations, si possible mirifiques (convaincre par la promesse)... Si un cahier des charges fonctionnel peut être réalisé, des travaux de recherche et de $R \& D$ peuvent permettre, après de nombreux efforts, de traiter de la complication du système pour arriver à un vrai démonstrateur (démarche algorithmique). Mais, comment aller au-delà de l'idée créatrice, de la disruption associée, de la preuve de concept pour atteindre des applications concrètes situées en dehors de l'accepté ?

A partir de la lecture des titres (et de nombreux résumés) des différents articles associés aux termes «4D printing » issus de la base CNRS, il a été possible de réaliser la figure 16. Plusieurs mots clés ont été retenus, correspondant à : 1- matériaux organiques et leurs stimulations ; 2Matériaux inorganiques et leurs stimulations ; 3 - Articles de synthèse ; 4- Modélisations ; 5- Divers ; 6- Applications et 7 : Procédés (dont les origamis 4D).

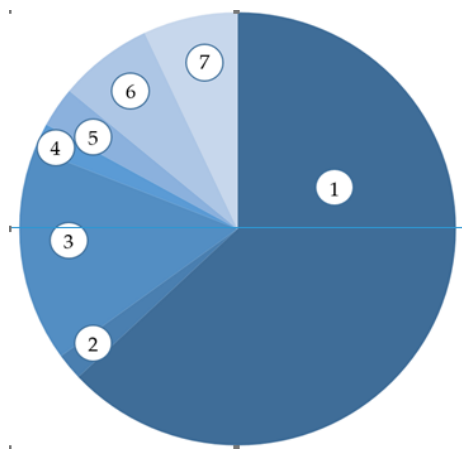

Figure 16. Thématiques couvertes dans les publications en impression $4 D$

(1: $63 \% ; 2: 2 \% ; 3: 16 \% ; 4: 2 \% ; 5: 3 \% ; 6: 7 \% ; 7: 7 \%)$

REMARQUE : Les applications traitent (hors bio-printing) de robotique souple, de chaussures 4D, d'applications en termes de capteurs et de délivrance de médicaments, de construction mécanique et d'optique.

Ce qui est très important à souligner, c'est la très grande unité des travaux centrés sur les stimulations de matériaux organiques purs (polymères) et chargés (en particulier avec des nanoparticules et des fibres), ce qui correspond à $63 \%$ des activités publiées. Mais si l'on retire $16 \%$ de publications de synthèse (pour un domaine en émergence où le nombre de publications se chiffre en quelques centaines), ce pourcentage devient $79 \%$ du total. Et quand on a retiré les documents divers et les applications, ce chiffre est encore plus élevé...

Ce constat conduit à l'implication prépondérante d'un domaine scientifique dominant constituant un ensemble de travaux dont l'applicabilité des résultats reste modeste et qui donc peut poser question (mais qui respecte la définition de l'impression 4D avec des matériaux adaptatifs avec des machines 3D)... Est-ce dû au fait que le couplage impression 3D - matière programmable ou stimulable ne peut se réaliser que sur ce type d'exemple ? Ou bien se situe-t-on dans l'exploitation du vieux principe dit du réverbère ?

Il existe quelques travaux sur des matériaux inorganiques qui se rapprochent en termes de performances mécaniques et de temps de réponse de ce qu'on peut exiger de la part de systèmes (actionneurs) réalisés par des voies classiques... Or, ceux-ci ne représentent que quelques $\%$ du total... Cette voie est-elle à privilégier, ou d'autres, exploitant d'autres paradigmes (qui seront discutés plus loin), d'autres visions (Impression 4D hétérogène par exemple : Jeong et al, 2019 ; Li et al, 2019 ; Zolfagharian, Kaynak et Kouzani, 2020 ; Ding et al, 2017) ou exploitaion des propriétés physiques et de conception des matériaux (Coulais et al, 2016 ; Hu et al, 2020) ? Ne doit-on pas 
favoriser une réelle créativité qui semble absente par un certain conservatisme et par des habitudes perpétuées ? Au fond, cette direction principale est-elle satisfaisante ?

Encadré 1 : Métaux et impression 4D

Ma et al, 2017 ; Kadic al, 2019 ; Lohmuller et al, 2019 ; Ambrosi, Webster et Pumera, 2020 ; Evans et al, 2020, etc. ont réalisé des impressions 4D de métaux à partir de machines spécialisées. C'est une des premières démonstrations d'utilisation de métal avec la publication de Lohmuller et al (2019). Dufaud, Marchal et Corbel, (2002) d'une part, Khim et al (2014) d'autre part avaient cependant déjà réalisé des impressions multi-matériaux pour l'un, avec des nanoparticules piézoélectriques pour le second.

Ces matériaux disposent de propriétés mécaniques satisfaisantes pour les applications, mais leur mise en forme est plus difficile (machines 3D plus coûteuses) et les processus de stimulation sont plus délicats à mettre en œuvre.

Voilà donc une tension qu'il faudrait guérir. « Nous fabriquons nos représentations dérisoires de ce monde mais toutes les structures que nous pouvons concevoir se situent dans nos représentations » (Hacking, 2001)... L'idée d'un domaine autonome et porteur de futur résiste difficilement à l'examen, mais ne peut s'effacer que devant l'idée non moins aporétique d'une science évanescente et ouverte aux quatre vents... (Utopie réaliste vs dystopie morbide ?).

\section{Pour sortir de l'impasse}

«La route en lacets qui monte. Belle image du progrès. Mais pourtant elle ne me semble pas bonne. Ce que je vois de faux, en cette image, c'est cette route tracée d'avance et qui monte toujours ; cela veut dire que l'empire des sots et des violents nous pousse encore vers une plus grande perfection, quelles que soient les apparences; et qu'en bref l'humanité marche à son destin par tous moyens, et souvent fouettés et humiliés, mais avançant toujours. Le bon et le méchant, le sage et le fou poussent dans le même sens, qu'ils le veuillent ou non, qu'ils le sachent ou non. Je reconnais ici le grand jeu des dieux supérieurs, qui font que tout serve leurs desseins. Mais grand merci. Je n'aimerais point cette mécanique, si j'y croyais [...]. Pour moi, je ne puis croire à un progrès fatal; je ne m’y fierais point » (Alain, 1962).

La fabrication additive est attrayante pour la recherche (en particulier après l'expiration des brevets de base). A l'évidence existent des problèmes importants d'organisation, d'intégration et d'impact réaliste de cette recherche. Pour Gao et al (2015), la raison principale tient à une recherche fragmentée dans laquelle les mécanismes d'intégration sont absents. Ce chapitre discute de ces éléments, applicables à l'impression 4D dans les paragraphes qui suivent. Il s'agit ici d'imaginer les mécanismes capables d'engendrer des solutions aux problèmes évoqués au paragraphe précédent. Plusieurs voies sont envisagées, l'une tirant son orientation sur des aspects scientifiques et technologiques, l'autre plus organisationnelle associée à des façons possibles de gérer les recherches émergentes.

Une définition complète de l'innovation pourrait être : «processus de traduction d'une idée ou d'une invention en un bien ou un service qui crée de la valeur avec des clients acceptant de la payer ». Pour qu'une idée soit qualifiée d'innovation, elle doit pouvoir être reproduite à un coût économique et répondre à un/des besoin(s) spécifique(s). "L'innovation implique l'application délibérée d'informations, d'imagination et d'initiatives pour tirer des ressources des valeurs plus grandes ou différentes, y compris tous les processus par lesquels de nouvelles idées sont générées et converties en produits utiles » (Mamasioulas, Mourtzis et Chryssolouris, 2020). Une innovation 
technologique disruptive est alors une innovation technologique qui finit par renverser (au moins une partie de) la technologie dominante ou le statuquo sur le marché. Pour l'instant, avec l'impression 4D, percée qui repousse des limites de la science et de la technologie pour sa mise en œuvre en fabrication, si la voie peut être au moins partiellement tracée, les clients ne sont pas encore atteints... on n'en est pas au développement et encore moins à la fabrication (Vielhaber et Stoffels, 2014).

La figure 17 proposée par Mamasioulas, Mourtzis et Chryssolouris (2020) représente une classification des types d'innovation de l'industrie manufacturière. Les domaines où l'impression 4D a une place sont indiqués par une couleur plus ou moins foncée selon son importance. Comme cette figure l'illustre, il y a une grande place potentielle pour le développement de cette technologie (qui n’a pas encore donné tout ce qu'elle pouvait)...

Innovations en relation avec la fabrication

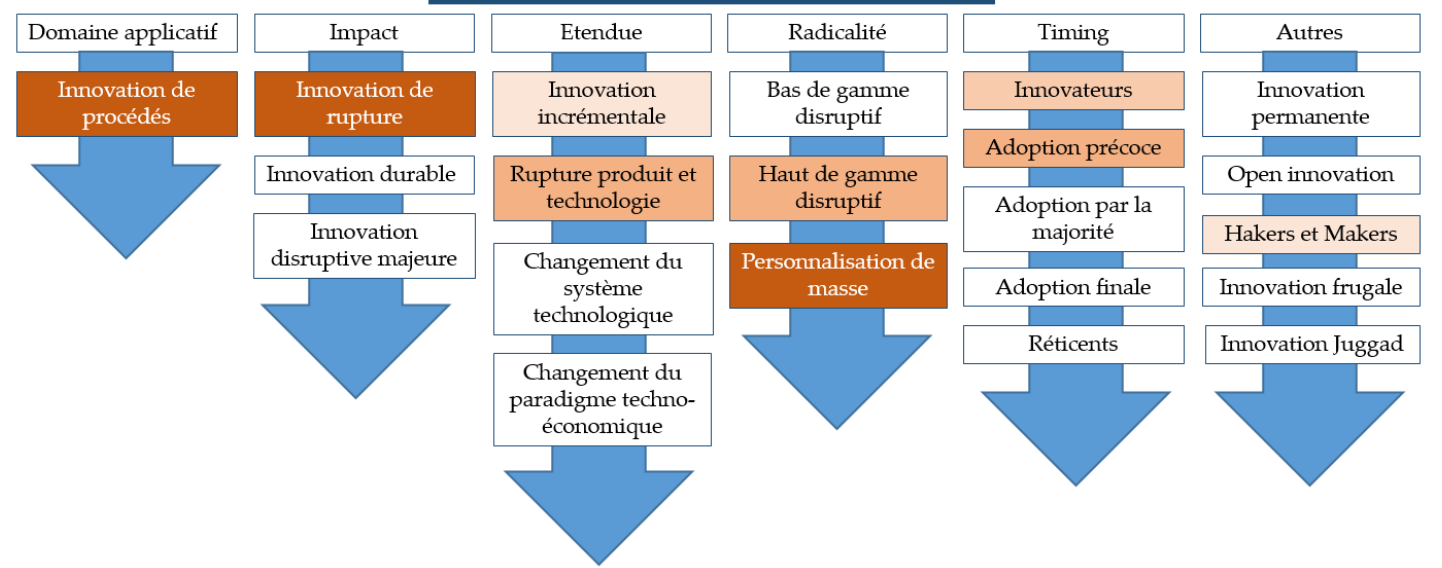

Figure 17. Classification des innovations en fabrication et place de l'impression 4D (couleurs)

Sur cette figure, on se situe dans la «théorie de la longue traîne » (Anderson, 2008) où l'on rend accessible des informations peu « marketées » rentrant dans des processus de dé-massification...

\section{REMARQUE : Compétences et maîtrise des technologies de fabrication}

L'utilisation d'une pluralité de technologies dans le secteur manufacturier est pratiquement obligatoire. C'est pourquoi le développement d'un nouveau processus comme l'impression 4D doit s'intégrer dans un ensemble de technologies disponibles (par exemple, les automates industriels, la mécatronique, les systèmes de fabrication flexibles, l'impression 3D, l'optoélectronique, etc.). Selon la taille des entreprises concernées et du potentiel humain, il est nécessaire de disposer de compétences couvrant tout le champ des outils disponibles, ce qui peut être difficile à gérer, surtout avec des technologies instables, en cours d'émergence...

\subsection{Aspects scientifiques et technologiques}

\subsubsection{Domaines scientifiques connexes}

La gamme des systèmes déformables est large, sans qu'une voie spécifique soit aujourd'hui privilégiée. Cependant, un certain nombre de problèmes scientifiques et technologiques restent à résoudre, soit à l'intérieur du concept strict d'impression 4D, soit en cherchant dans des domaines proches pour examiner si la porosité existante entre domaines technologiques peut permettre d'envisager des suites plus favorables au développement applicatif promis. Or, dans les domaines de la robotique souple et de l'actuation se développent depuis plusieurs années des travaux proches de la fabrication 4D. Il peut s'agir de l'utilisation d'origamis, de matériaux dont un au moins est adaptatif ou de l'usage de méta-matériaux ou de principes mécaniques souples. 
Cette expression d'Extrême Orient s'invite ici parce que l'on peut donner une forme à trois dimensions à une structure initiale qui est plate sur laquelle on peut imprimer des matières actives (passage de 2D à 4D). Pour des entités de taille au moins centimétrique, il est possible de positionner un actionneur classique à certains plis ; leur rotation permet le mouvement des plans et par suite le déplacement de l'origami qui peut, par une commande adaptée, devenir un robot-origami (Nisser et al, 2016, Ackerman, 2016).

La figure 18 dont le principe est issu de Ge et al (2014) reprend la même idée avec des systèmes thermomécaniques. Dans leur publication les temps de relaxation sont de l'ordre de 0.4 seconde. Sur un design adapté, il y a possibilité d'atteindre des formes plus complexes pour autant que l'objet puisse se décrire sur un plan avant d'être plié (Abtan, 2019; Zaghloul et Bone, 2020 ; Callens, Tümer et Zadpoor, 2019).

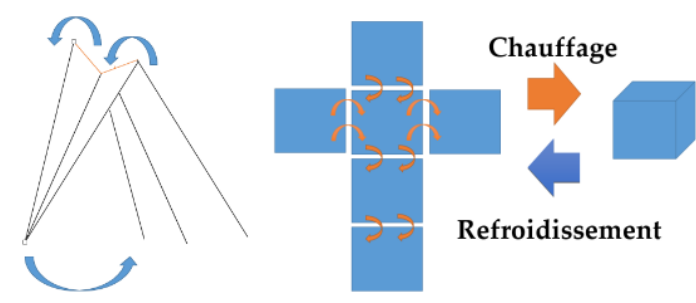

Figure 18. Principe de fonctionnement d'un origami thermomécanique

\section{REMARQUE : Kirigamis (Jian et al, 2019 ; Aharoni et al, 2018)}

Il s'agit d'une extension en 2D des origamis avec des changements selon des directions privilégiées imposés par une stimulation conduisant par exemple avec des matériaux élastiques au passage d'une plaque à un cône.

\section{Méta-matériaux}

La complexité structurelle des méta-matériaux est en principe illimitée, mais la plupart des conceptions comprennent des architectures périodiques qui conduisent à des matériaux présentant des caractéristiques spatialement homogènes (Coulais et al, 2016). Le flambage, qui était autrefois considéré comme le «summum » de l'échec de conception, a été exploité au cours des dernières années pour développer des méta-matériaux mécaniques aux fonctionnalités avancées permettant de créer des structures tridimensionnelles à propriétés exploitables en impression 4D (Janbaz et al, 2019 ; Cui, Smith et Liu, 2010 ; Cui et al, 2014 ; Buriak et al, 2016 ; Coulais et al, 2016 ; Krödel et al, 2017 ; Jayashankar et al, 2019 ; Hahn et al, 2020). La figure 19 (Novak, Vesenjak et Ren, 2016 ; Ion et al $(2016 ; 2018 ; 2018$ a) illustre sur ces bases la possibilité de réaliser des structures souples qui peuvent être mises en mouvement en utilisant des actionneurs mécaniques externes ou internes. Ces structures peuvent être réalisées en impression 3D. Ainsi, contrairement à l'impression 4D homogène, on utilise des structures filaires ou des mousses (et non un matériau en volume) avec des actuations externes (rappelons cependant que les matériaux doivent être suffisamment souples pour envisager la déformation, mais peuvent n'être que « passifs »). 


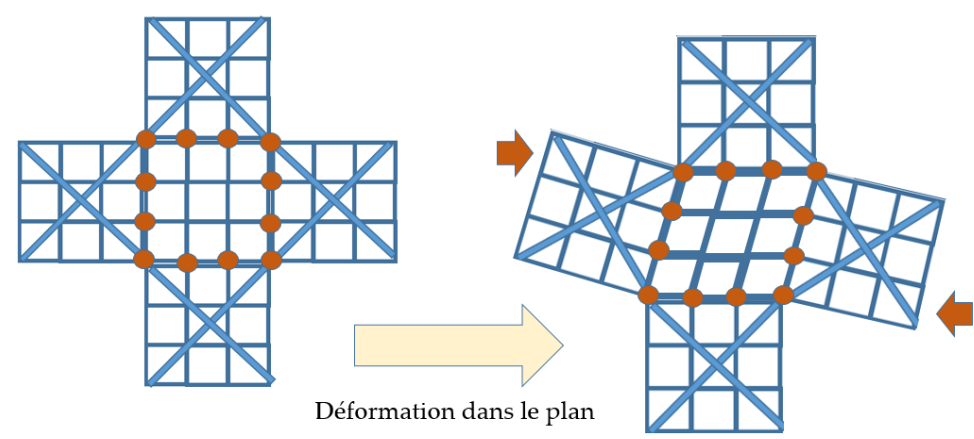

(A)
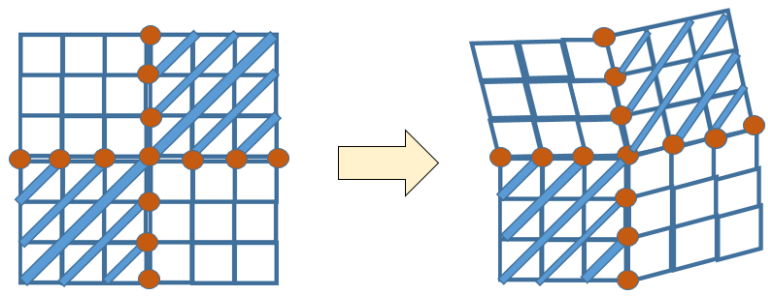

Déformation hors du plan

(B)

Figure 19. Déformation de structures hétérogènes sous l'action d'une force externe (les disques en rouge correspondent à des points d'attaches entre les différentes structures : A : Déformation planaire ; $B$ : déformation spatiale à partir d'une structure plane)

\section{Actionneurs souples}

Il peut s'agir comme le montre la figure 20 de solutions simples et traditionnelles d'actionneurs qui peuvent être réalisés par fabrication additive (Peele et al, 2015). Sur cette figure, le déplacement à gauche ou à droite dépend de la différence de pression gazeuse entre les deux compartiments de l'actionneur.

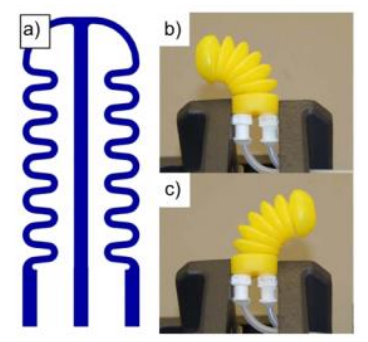

Figure 20. Actionneur 3D pneumatique

Dans le même esprit, les travaux de Walters, Rossiter et McGoran (2010) ; Hwang, Paydar et Candler (2015); Gong et al, 2016; de Jeong et al (2019); Li et al (2019); Lau (2019); Zolfagharian et al, 2019 ; Zolfagharian, Kaynak et Kouzani (2020); Tang et al (2020) conduisent à des pinces ou à des actionneurs utilisables en robotique. Le principe de fonctionnement est le même que celui utilisé par Peele et al (2015).

\section{Conclusion}

Ce que montrent ces résultats synthétiques, c'est qu'il existe des voies de déploiement du thème 4D vers l'utilisation de matériaux connus, présents « sur étagère », mais dont l'organisation spatiale peut servir à la réalisation d'objets disposant de formes souples ou de fonctionnalités nouvelles. 
Alors, pourquoi ne pas déplacer le centre d'intérêt de la recherche vers l'utilisation de ce type d'organisation spatiale ? Le tableau 4 tente de faire une comparaison entre ces deux champs de possibilité, entre $4 \mathrm{D}$ printing homogène et ses ouverture qualifiées de $4 \mathrm{D}$ printing hétérogène.

\begin{tabular}{|l|l|l|}
\hline Thème & Commentaires 4D homogène & Commentaires 4D hétérogène \\
\hline Matériaux & Massifs & Filaires ou surfaciques \\
\hline $\begin{array}{l}\text { Propriétés } \\
\text { mécaniques }\end{array}$ & Aujourd'hui insuffisantes & Adaptées \\
\hline Actuation & Dans le matériau lui-même & $\begin{array}{l}\text { Externe avec des dispositifs additionnels. } \\
\text { Compte tenu des proriétés mécaniques } \\
\text { des matériaux 4D homogènes, il n'y a pas } \\
\text { (ou peu) de travaux où l'on utilise les } \\
\text { propriétés actives des matériaux en } \\
\text { particulier polymères }\end{array}$ \\
\hline Stimulation & Externe & Externe ou interne \\
\hline Modélisation & Difficile dans des matériaux massifs & $\begin{array}{l}\text { Possible avec des systèmes filaires, plus } \\
\text { délicat avec des éléments surfaciques }\end{array}$ \\
\hline $\begin{array}{l}\text { Résolution spatiale } \\
\text { des propriétés }\end{array}$ & $\begin{array}{l}\text { Difficile, sauf avec des systèmes multi- } \\
\text { matériaux }\end{array}$ & Adaptée \\
\hline Problème inverse & Très difficile & Difficile \\
\hline
\end{tabular}

Tableau 4. Comparaison entre les deux formes homogène et hétérogène de fabrication $4 D$

\subsubsection{Le problème inverse}

«L'innovation jouit d'une aura et d'un prestige sans précédent qui n'entretient qu'un rapport
ténu avec les réalités économiques » (Fressoz, 2018).

La figure 21 rappelle la finalité de l'impression 4D avec deux opérations associées interdépendantes : la fabrication d'un objet par une technologie 3D et l'activation de ce dernier. Avec par exemple un actionneur, on peut définir un cahier des charges fonctionnel et, en examinant la liste des matières et/ou des matériaux actifs utilisables en fabrication additive, les machines 3D accessibles, définir de manière intuitive un dispositif simple qui répondra à la demande. En revanche, avec des objectifs plus compliqués (robotique souple, par exemple), la situation devient plus problématique : où doit-on placer les matériaux actifs ? Comment les stimuler et avec quels dispositifs? Etc. A cette date, on ne sait pas s'il existe une, zéro ou une infinité de solutions. La question du problème inverse est une question en soi, et elle n'a pas fait l'objet d'un traitement conceptuel à ce jour.

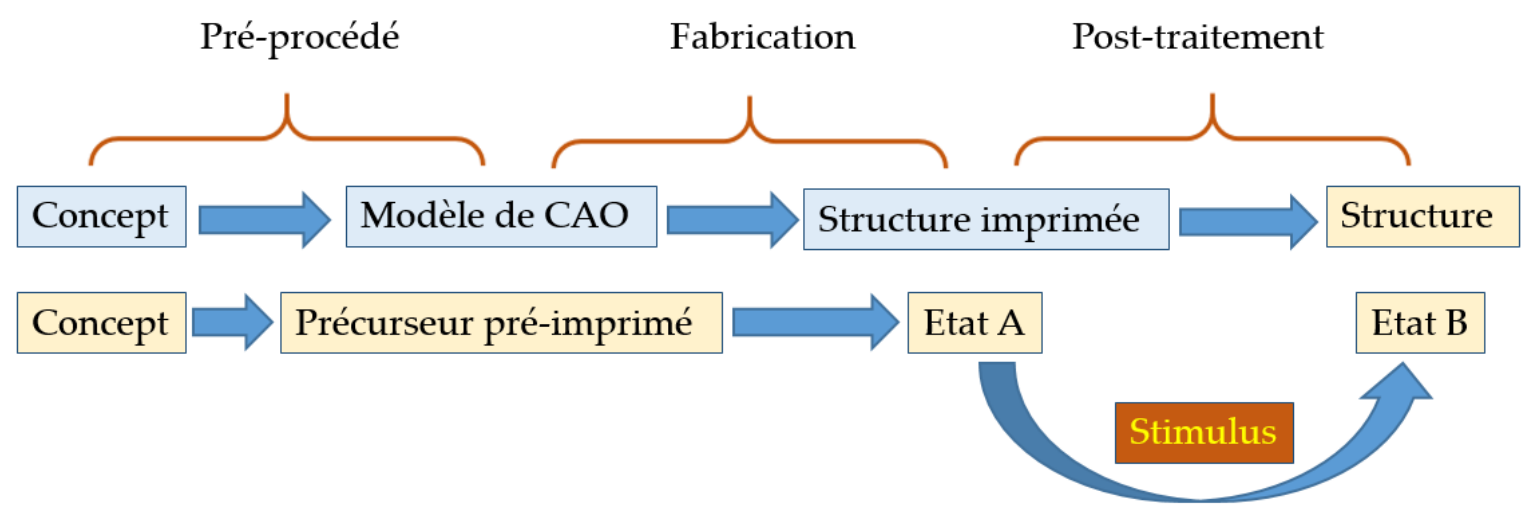




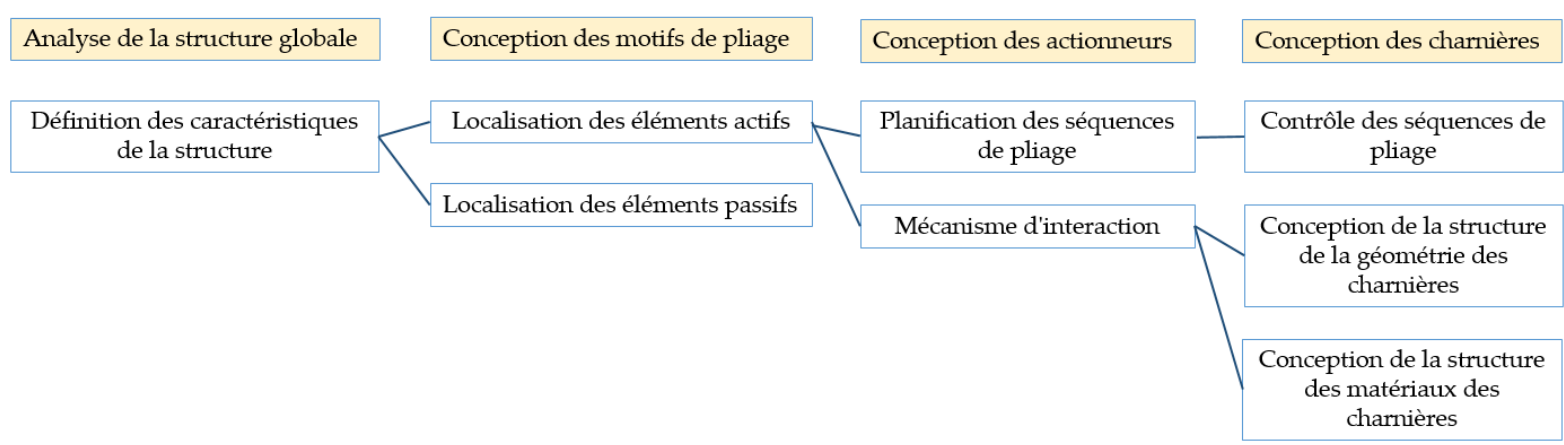

Figure 21. Couplages Fabrication - actuation en impression 4D (avec un grand nombre de possibilités de changements de forme - figure du haut : cas général ; figure du bas : origamis)

\subsection{Aspects organisationnels (et financiers)}

«La croyance heuristique est ainsi celle qui consiste, pour l'auteur du projet, à éprouver dans le temps, à la fois la viabilité de son projet et le sérieux de son intention, là où l'hypothèse, qui formalise une interprétation ou un schème explicatif, n'est pas nécessairement corrélée à une intention, un souhait de réalisation » $(\mathrm{Nal}, 2013)$.

Les solutions «satisficing »(Simon, 1976), pour décrire celles dont on se satisfait, faute de pouvoir en pratique déterminer une solution optimale correspond peu ou prou, pour le moment, à ce que la recherche peut proposer pour l'impression 4D. Il s'agit de solutions acceptables via une " recherche heuristique », une recherche sélective tâtonnante utilisant des connaissances acquises (d'où certains conservatismes). Il n'y a pas, à la connaissance des auteurs, de recherche opérationnelle conduisant à l'utilisation d'algorithmes de calcul d'une solution optimale, avec réduction du problème pour qu'il puisse satisfaire les contraintes de modélisation (Avenier, 2019). Un certain flou règne donc, laissant une large place à l'imagination, pour autant qu'elle existe et qu'on sache la promouvoir.

\subsubsection{Cadre général}

«Jusqu'à la classe de terminale, je me représentais la science comme un jardin à la française, où tous les chemins étaient soigneusement tracés » (Monod, 1997).

Ce qui parait important aux deux auteurs, c'est la finalité ; l'impression 3D est alors un moyen pour atteindre un objectif de réalisation d'objets actifs dont on peut imaginer un grand nombre d'applications. Alors, dans un monde gouverné par des soutiens financiers orientés, la demande concerne l'existence d'un pouvoir chargé de l'organisation et du financement de la recherche qui soit « philosophiquement» neutre, ne cherchant pas a priori à imposer une conception, sous la seule réserve que les choix soient éclairés à l'intérieur de règles communes (choix des experts, accords sur les prises de risques, par exemple). Pour autant, il ne s'agit pas de se réfugier dans un budget global «fourre-tout » de type PIB (Michéa, 2007). Ce cadre spinoziste, forme d'intelligence de la nécessité avec une stratégie du «moindre mal », rappelle cette phrase de Benjamin Constant qui écrivait : «Prions l'autorité de rester dans ses limites, qu'elle se borne à être juste, nous nous chargeons d'être heureux »! Orwell (2008) parlait de « société décente » (common decency). Il s'exprimait pour une société dans laquelle chaque acteur rationnel aurait la possibilité de vivre honnêtement d'une activité qui ait réellement un sens humain. Il est vrai que ce critère apparemment minimaliste implique déjà une réduction conséquente des inégalités. Où est, toutes choses égales par ailleurs, la «common decency » en recherche, sans qu'il soit question de soutenir à tout prix l'apologie de la transgression? 
Une communauté 4D ouverte, faisant abstraction de certitudes inadaptées, doit être un regroupement pour le partage d'une passion commune avec des interactions régulières et le partage des meilleures pratiques dans la communauté pour faire émerger des idées nouvelles. Cette créativité nécessaire dans un domaine en pleine émergence doit être considérée comme une source d'innovation. La coopération nécessaire passe probablement par de nouvelles méthodes de management (Sarazin, Cohendet et Simon, 2017). Si les contraintes initiales sont trop rigides, de nombreuses directions de développement possibles seront fermées par un projet général ne laissant pas de place aux initiatives et il sera difficilement possible que les objectifs non inclus dans l'ensemble initial exercent une grande influence sur la conception finale (Simon, 1995). Il faut donc savoir ce que l'on veut... Anticiper ou suivre ! Prendre des risques ou pas !

D'ailleurs, il est déjà difficile de convertir les nouveaux concepts technologiques en produits commercialisables et de faire passer les projets de la découverte au développement de produits nécessite le passage de ce qu'on appelle la vallée de la mort. Si le soutien de l'Etat n'est pas présent à ce stade ultérieur, cette lacune ne peut être comblée (Dedehayir, Mäkinen et Ortt, 2018 ; Russell et Smorodinskaya, 2018). Dean, Zhang et Xiao (2020) montrent que la complexité organisationnelle et la complexité du produit interagissent avec les turbulences du marché sur la transition via cette vallée)... D'où la nécessité d'un effort et d'un soutien à la prise de risque. Pour les chercheurs académiques, c'est, quand cela fonctionne, le rôle dévolu aux SATT (Sociétés d'Accélération du Transfert de Technologies)... Mais, comme elles doivent prouver leur rentabilité à court terme, il faut trouver un chargé de «clientèle» disposant de valeurs fortes pour apporter ce soutien nécessaire.

Comme dans le cas de la fabrication additive, l'impression 4D peut être présentée comme un élément d'une nouvelle révolution industrielle, dans laquelle la numérisation, l'information, la flexibilité et la connectivité transforment l'innovation en matière de produits. Si les avantages offerts peuvent convaincre, les recherches actuelles suggèrent que les promesses des technologies 4D sont rarement vérifiées dans la pratique. Il faut par un soutien éclairé démontrer que l'adoption des procédés 4D apporte de réels avantages aux entreprises (concepteurs et utilisateurs). Dans la situation de désordre associée à des crises (Samra et al, 2019; Mac Causland, 2020) comme celle liée au coronavirus, avec un environnement confronté à l'incertitude, la fabrication 4D qui est une technologie numérique, devrait être soutenue, pour autant que côté recherche, le monde académique et celui du transfert aille effectivement vers autre chose que des preuves de concept spectaculaires, mais industriellement peu utiles.

Beltagui, Sesis et Stylos (2019 proposent, dans un autre cadre, de tenter de comprendre comment l'impression 4D est utilisée par des innovateurs indépendants (Fab-Lab par exemple), dans le contexte des espaces de production, pour générer des innovations. Le Fab-Lab permet à des communautés l'accès à ces nouvelles technologies, d'apprendre à les utiliser et de développer leur capital social, avec pour objectif :

- De comprendre les motivations des innovateurs qui utilisent la 4D et les espaces de production ;

- De disposer d'informations sur le rôle que l'impression 4D et les espaces de production peuvent jouer dans l'innovation commerciale.

Cette interface active jouant avec du «bricolage savant» est une approche de l'innovation qui met l'accent sur l'expérimentation, l'improvisation et la mise en réseau pour surmonter les contraintes liées aux ressources. Elle peut être d'une redoutable efficacité (André, 2017). Dans un premier temps, cette activité permettrait de démontrer que l'impression 4D peut être utilisée pour combler des lacunes, en créant des pièces non standardisées ou autrement inaccessibles, en combinaison avec d'autres ressources disponibles. Le Fab-Lab pourrait ainsi jouer un rôle dans le partage, de métissage des connaissances, pour contribuer à l'innovation et son intégration dans la société (comme pour la 3D selon Joseph et Holm, 2015 ; Heemsbergen et al, 2019). Saura-t-on 
comme dans le cas de la fabrication additive intégrer l'impression 4D dans la chaîne de valeur industrielle ? (Durach, Kurpjuweit et Wagner, 2017 ; Hannibal et Knight, 2018 ; Lam et al, 2019 ; Kunovjanek et Reiner, 2020 ; Culot et al, 2020). Dans ce domaine, une augmentation de la relation avec les clients avec la possibilité de co-création se manifeste avec un effet sur les ventes par le biais du prototype réel du produit commercialisé ou par un soutien technique à long terme (Godina et al, 2020).

\subsubsection{Gérer l'interdisciplinarité et la complexité}

«C'est le meilleur directeur de toute l'Allemagne, la totalité de l'orchestre semble sortir de sa baguette » (Mendelssohn, 1999).

Maslow (1966) écrivait : «Un marteau tend à voir tous les problèmes sous forme de clou »! La créativité qui est souvent mise en avant avec ses nombreux clichés ou images d'Epinal constitue un point de départ obligé à l'innovation, en particulier, celle issue de la recherche explorant des approches téléologiques, parce qu'elle force à penser autrement à l'intérieur de systèmes de plus en plus complexes et incertains. Cette situation devenue urgente, sans être nouvelle sur le fond, induit un phénomène nouveau dans les structures de recherche, en particulier académiques, celui de la prise de risque d'échec (mais en cas de succès, celui qui arrive le premier gagne tout, surtout s'il est déjà reconnu par ses pairs et sa hiérarchie...). Or, à l'évidence, les recrutements des chercheurs se font sur une aptitude à approfondir (avec des taux de sélection très réduits ; la compétence scientifique de ces derniers n'est donc certainement pas en cause) avec des soutiens financiers qui sont définis (pour faire simple) par des personnalités ayant montré leur pertinence dans l'ancien monde qu'il s'agisse du niveau national ou européen. L'incrémental règne donc. S'il n'est pas question de supprimer ces voies d'accès à des formes de progrès, les auteurs estiment nécessaire de mieux soutenir ces «empêcheurs de penser en rond » que sont les créatifs, qui de toutes les manières auront besoin, pour mener leur idée à un terme «vendable », de spécialistes affirmés, ceux qui explorent aujourd'hui la continuité et l'exploration sérieuse de paradigmes scientifiques.

La créativité peut être considérée comme « l'habileté à transformer les idées neuves et pleines d'imagination en réalité » (Blanc, 2019), le développement de compétences créatives dans le cadre des sciences de l'ingénieur sont essentielles, cruciales et, dans le contexte dessiné, finalement indispensables. Cependant, l'ingénierie a été surtout perçue comme un domaine prenant appui sur des connaissances mathématiques et technologiques disciplinaires (Stouffer, 2004). Il y a donc nécessité de sortir du confort douillet des avancées incrémentales (certes utiles, mais peu productrices de remises en cause, car ces approches dépendent trop de la ré-application de connaissances factuelles (Cropley, 2015)).

\section{L’impression 4D repose-t-elle sur la complexité ?}

« Il n'y a pas d'idée simple, parce qu'une idée simple [...] doit être insérée, pour être comprise, dans un système complexe de pensées et d'expériences » (Bachelard, 2003).

La façon de se positionner face à l'idée de complexité renvoie au degré de fermeture (ou d'ouverture) attribué à la définition de ce qui est complexe : est-ce de l'hyper-complication (s'l s'agit d'une check-list elle perd toute spécificité) ou est-ce réellement complexe ? Entre ces deux extrêmes, la définition de la notion implique des processus de négociation des significations qui s'y rattachent, ajoutant ce faisant des ambiguïtés (Alhadeff-Jones, 2008). Pour les auteurs, la complexité engendre des changements sur nos modes de tentatives de maîtrise du monde réel : Le premier concerne le statut de la réalité à l'exemple de la dualité onde-particule, le second concerne l'articulation des disciplines sur lesquelles s'appuie la réflexion complexe (cf. convergence et interdisciplinarité) (Piaget, 1967 ; Atlan, 1979 ; Araujo-Jorge, 1991 ; Beguin et Cerf, 2009 ; Le Moigne, 2001 ; Donnadieu et al, 2003 ; CNRS, 2002 ; Mathieu et Schmid, 2014 ; Simon, 2004, 
etc.). Selon ces auteurs, la systémique est une « discipline qui regroupe les démarches théoriques, pratiques et méthodologiques, relatives à l'étude de ce qui est reconnu comme trop complexe pour pouvoir être abordé de façon réductionniste, et qui pose des problèmes de frontières, de relations internes et externes, de structure, de lois ou de propriétés émergentes caractérisant le système comme tel, ou des problèmes de mode d'observation, de représentation, de modélisation ou de simulation d'une totalité complexe ». La figure 22 rappelle quels sont les différents éléments à prendre en considération dans une approche système.

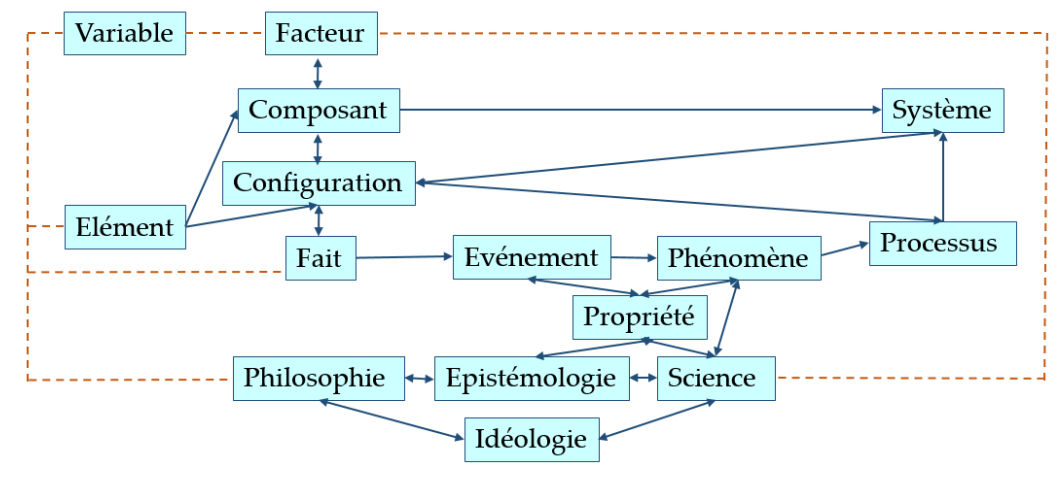

Figure 22. Approche système : catégories des description d'état et de processus

\section{Gestion de projet interdisciplinaire}

«La recherche à la française est une aventure solitaire à l'intérieur d'un système démocratique » (Crozier et Tillette, 2000).

Abordé au $\$ 2.1 .1$, ce thème pose la question du recrutement de personnes motivées engagées dans l'action. Cette délicate opération menée, différentes méthodes ont pour mission de renforcer les liens entre les différents acteurs et par suite, de gagner du temps dans la transition entre l'idée et la réalisation d'un projet via une étape de conception. Pour Barnard (2017), une règle classique est d'examiner si une solution envisageable a plus de poids dans l'une des disciplines convoquées, mais égale dans tous les autres, cette solution dominera probablement. Des hiérarchies peuvent alors s'exprimer. Mais, à l'évidence, il n'y a pas une méthode unique, même si la plupart de celles-ci impliquent la création d'un groupe projet (cf. figure 23) et la définition de liens entre les différentes étapes (cf. figure 24, issue de Marsot, 2002), en tentant de bien séparer chaque étape de l'action, constituant un noyau d'indépendance provisoire.

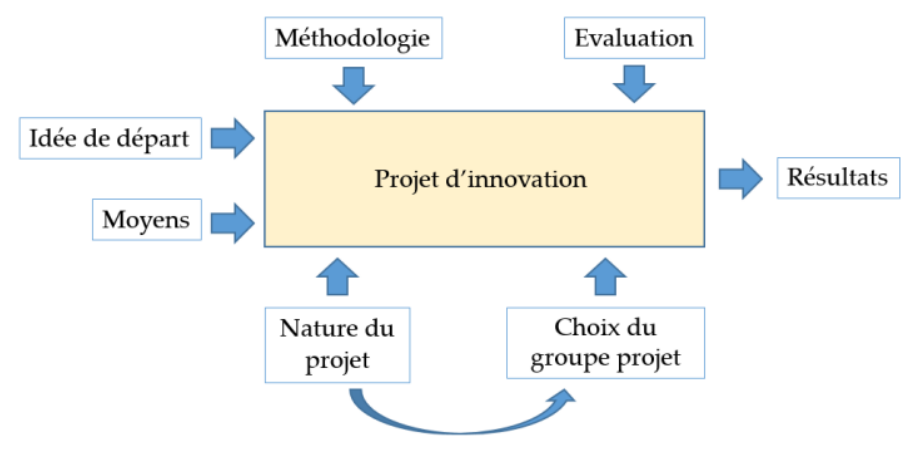

Figure 23. De l'idée au résultat via l'étape de conception

Dans ce projet représenté par un rectangle sur cette figure se placent différents éléments qui définissent selon Roozenburg et Ekels (1995) le cycle élémentaire de la conception avec une voie royale (quand tout marche) et des retours, voire des arrêts quand des problèmes émergent. La figure 24 illustre ce principe qui est une base organisationnelle (parmi d'autres). De plus, des rejets et du travail déjà réalisé peuvent émerger de manière latérale d'autres idées. 


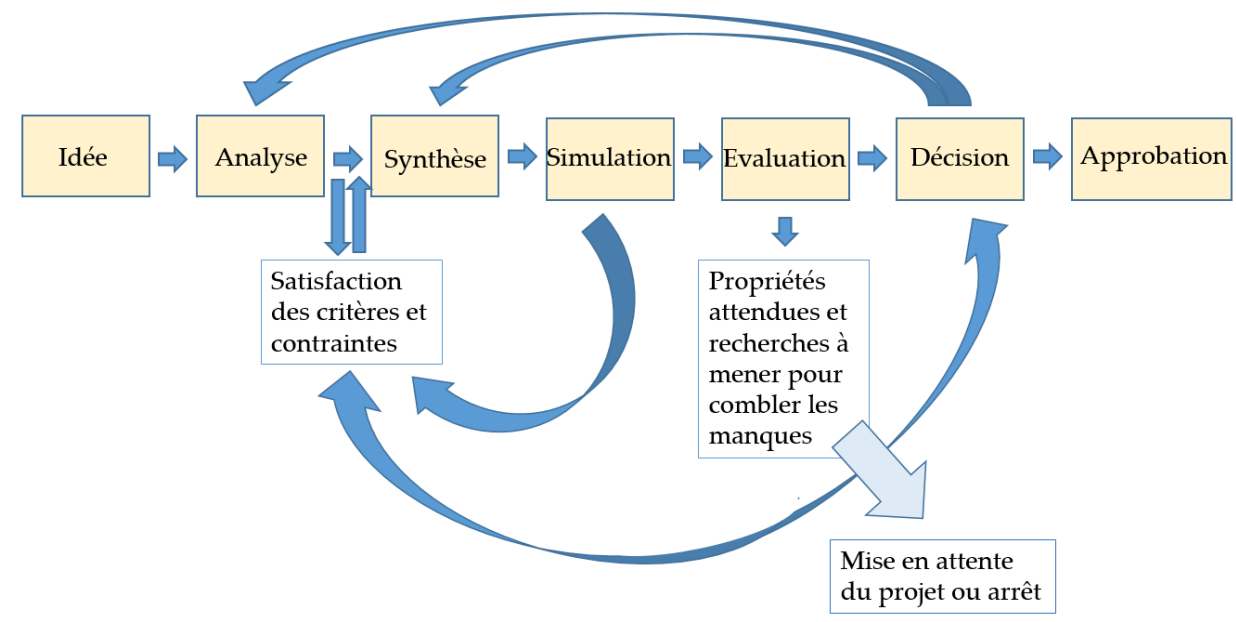

Figure 24. Cycle élémentaire de la conception

\subsubsection{Sortir de l'incrémental...}

« On ne découvre pas de terre nouvelle sans consentir à perdre de vue, d'abord et longtemps, tout rivage » (Gide, 2008).

L'information est présente partout (ou presque) avec Internet, mais n'est pas ou peu appliquée dans les unités de recherche qui restent généralement sur des activités d'innovation incrémentale (financements plus accessibles, reconnaissance par les pairs, etc.). Or, ce processus requiert la mise en forme de l'information qui doit être adéquate pour répondre au besoin : d'où la résolution de cette question, sa planification, le tri des résultats (et de leur robustesse) et fourniture d'un document adapté au destinataire (Goria et Levassor, 2015; Goria, 2019). C'est bien comme cela que se remplissent les propositions à des appels d'offres.

Entre l'idée initiale et l'accomplissement de cette idée en termes conceptuels et/ou applicatifs, un passage souvent obligé consiste en la constitution d'un groupe disposant de personnalités avec des qualités hétérogènes, mais associables et qui doivent s'entendre en vue d'un objectif mutualisé. La créativité peut être personnelle, même si des choses créatives peuvent parfois partagées et avoir un impact sur l'innovation (Runco, 1995, 1996, 2015 ; Runco et Jaeger, 2012 ; Corraza, 2015). Le contenu de l'activité d'innovation peut être séparé en deux étapes principales :

- L'étape de créativité initiale, qui comprend l'émergence de l'idée et la préparation d'un plan d'exploration associé à la poursuite de la mise en œuvre de l'activité, avec des ajustements ultérieurs. Elle débute par l'émergence de l'idée de l'initiateur suivie du recrutement des « bonnes personnes » et de la justification des ressources nécessaires à la réalisation pratique et/ou conceptuelle de l'idée. La diversité des disciplines convoquées et des cadres de référence amènent de la flexibilité, une capacité d'adaptation, de la créativité et de l'innovation (Jehn, Northcraft et Neale, 1999). C'est en fonctionnant que le groupe «projet » fera émerger des besoins d'approfondissement, de clarification, de remise en cause de l'idée, des bifurcations, etc.

- La deuxième étape peut correspondre à une activité moins innovante, car s'appuyant sur l'exploitation de l'art antérieur scientifique et/ou technologique. Les aspects économiques peuvent aussi intervenir dans ce processus. L'approche interdisciplinaire proposée tient compte non seulement de toutes les composantes et, dans un espace temporel contraint, de l'évaluation a priori de l'efficience, mais aussi des caractéristiques psychologiques des créateurs d'idées, du groupe et du support de l'environnement institutionnel (Mynasian, 2018)... 


\section{REMARQUE : heuristique}

Selon Dumont (2018) : «L'heuristique (du grec heuriskêin, « trouver ») est l'utilisation de règles empiriques : pratiques, simples et rapides, facilitant la recherche des faits et l'analyse de situations, dans un objectif de résolution de problèmes et de prise de décision, dans un domaine particulier ». La figure 25 qui représente le champ d'une expertise formelle ou non permet de trouver une place spécifique à la créativité prenant sa pleine dimension dans l'existence de domaines qui sont encore à explorer. C'est bien dans l'exploration heuristique du « lac de non savoir » que les risques émergent...

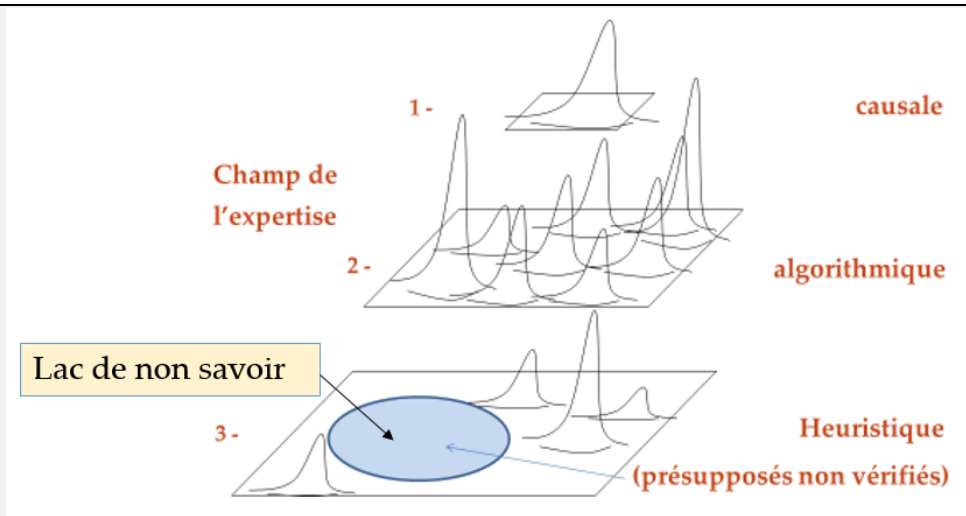

ELARGISSEMENT DU CHAMP DE L'EXPERTISE

Figure 25. Champs d'expertise (les gaussiennes approximatives représentent les acquis scientifiques issus du travail de recherche antérieur; selon les formes d'expertise, le domaine est couvert (causal, algorithmique) ou partiellement couvert (heuristique)) (André et Masse, 2002)

Relativement aux modes classiques d'innovation, le cahier des charges se construit «en marchant » avec de possibles remises en causes et de possibles risques d'échecs... Pour Holm et al (2013), les comités d'experts en charge d'évaluer les projets de recherche ne seraient pas toujours, de par leur composition, réellement aptes à évaluer des projets interdisciplinaires projets et, dans ceux-ci, à reconnaître ceux qui sont potentiellement innovants... (Voir également Dillaert, 2014).

\subsubsection{Transfert de technologie - Relier l'académie au monde réel}

« Les chercheurs industriels agissent sous l'autorité d'un gestionnaire plutôt qu'à titre individuel. Leurs recherches sont commandées pour atteindre des objectifs pratiques, plutôt qu'entreprises dans la poursuite de connaissances. Ils sont employés comme experts en résolution de problèmes, plutôt que pour leur créativité personnelle [...]. La notion d'« industrialisation » de la science universitaire signifie donc quelque chose de plus que le fait que certaines institutions sont prises en compte dans le secteur privé de l'économie nationale. Elle implique l'établissement au sein de la science académique d'un certain nombre de pratiques essentiellement étrangères à sa culture » (Ziman, 2000).

Si l'on parle de technologie d'impression 4D, cela signifie que l'on est capable de proposer des «procédés bien définis et transmissibles destinés à produire certains résultats jugés utiles » (Friedman, 1952). Ce contexte impose donc de définir des règles claires sur les modes de fonctionnement relativement à une fin applicative, donc d'une traduction des possibles complexités évoquées ci-dessus en termes de réductions permettant l'usage. 


\section{REMARQUE :}

Une nouvelle technologie, à l'exemple de la fabrication additive, n'épuise que très rarement et de manière complète celle qui lui a précédé. Par ailleurs, pour différentes raisons présentées dans cet article, seuls quelques aspects radicaux caractérisent l'innovation, les autres composantes de cette dernière puisant leurs savoirs de la connaissance préexistante. Mais, comme la tendance des milieux industriels est plutôt attentiste pour différentes raisons (complexité, difficulté d'avoir, en même temps, dans la même organisation des technologies concurrentes avec les formations spécialisées, financements à opérer, etc.) l'innovation risque de se développer ailleurs. De manière évidente, il existe un réservoir pour cette réalisation, celui de la recherche académique, qui n'est pas réellement adapté à ce type de processus (approche disciplinaire, évaluation spécifique, etc.).

Dans le domaine de la conception et de l'innovation de rupture, la complexité surdétermine l'objet technique, car son intégration dans son environnement futur doit être prévue. L'objet actif doit être inclus dans un cadre de développement plus large : désirabilité, modes de production, d'utilisation, relations clients et utilisateurs, parties prenantes, création de valeur, etc. (Lizarralde, Larrasquet et Coutts, 2011). Par ailleurs, les objets technologiques sont de plus en plus dépendants des services associés et des contenus fournis par des tiers, à titre gratuit ou commercial. Une particularité de ces services technologiques est 1'ignorance du client face à des technologies nouvelles, et des services inattendus, dans lesquels il ne peut se projeter avant de les avoir essayés, et pour lesquels il doit découvrir et inventer de nouveaux usages (Salgues et Bister, 2014). Ces considérations échappent normalement au chercheur académique.

La conception 4D doit être considérée comme partant de plusieurs mots clés : initiative, créativité, responsabilité, confiance, sens, partage, processus, flexibilité, culture, apprentissage, etc., ces domaines contribuent à ajouter de la complexité... De surcroît, la fabrication 4D qui génère une inséparabilité technologique supérieure à l'impression 3D doit produire un objet actif issu de l'imprimante plutôt que par activités organisées en différents stades successifs. Il y a donc des compléments informatifs à opérer. En tout état de cause, des axes communs d'actions doivent/peuvent conduire à un transfert comme (Leleu-Merviel et Boulekbache, 2014) :

- Peut-on restituer l'idée, son traitement depuis sa complexité grâce à une forme synthétique de formulation utilitaire?

- Comment conceptualise-t-on cette formulation ? Et comment représente-t-on ce qu'on a conceptualisé et/ou conçu ?

- Les outils de cette représentation du projet interdisciplinaire influencent-ils en amont le fonctionnement du groupe projet?

- Quel est l'effet des représentations en conception incrémentale ou de rupture sur les échanges entre les acteurs : réductionnisme, standardisation?

- Existence d'outils pour une meilleure compréhension des intentions de conception?

- Définition d'un modèle de l'activité de conception ? Et si oui, comment ?

L'industrie avec l'impression numérique passe d'une logique de production de masse à celle de personnalisation de masse (Blanchet, 2016). Elle ne repose plus sur les effets d'échelle et de volume, mais sur une production flexible et localisée près de la demande, ce qui peut être parfaitement adapté à l'impression 4D. Pour Frigant (2020), un fournisseur s'équiperait d'une imprimante 4D (machine et réactifs) pour répondre à la demande spécifique d'un client souhaitant disposer de pièces aux formes et à des fonctionnalités inédites. Ou bien, un fournisseur s'équipe d'une gamme de productions proposée au marché. Dans les deux cas, il y a création en local d'une 
compétence spécialisée comme cela est le cas pour l'impression 3D. Mais, les spécialistes devraient probablement revenir aux principes de base du «storytelling » pour engager de futurs utilisateurs : le développement du domaine 4D devrait faire appel à la curiosité des utilisateurs, ce qui suppose au-delà des aspects techniques une information adaptée pour interagir. «Les publicités les plus puissantes d'aujourd'hui retardent le plus longtemps possible le moment où le consommateur se rend compte qu'il s'agit d'une publicité » (Hally, 2020).

\subsubsection{Evaluation}

«Il est clair qu'un certain nombre de nos choix sont dictés par des considérations sur le jugement de nos pairs et leur comportement » (Tirole, 2016).

«Les décideurs politiques [devraient] être très conscients des conséquences involontaires de l'évaluation de la recherche et préparer des actions de contrepoids. La conséquence la plus risquée est peut-être la bureaucratisation. La lente transformation de l'évaluation de la recherche en une activité administrative de routine est dangereuse. Une autre conséquence non intentionnelle est l'adoption de comportements stratégiques ou de jeux au point de mettre en danger les valeurs académiques » (Bonaccorsi, 2020). Pour l'essentiel, ce sont des considérations disciplinaires qui sont impliquées dans les processus d'évaluation de la recherche, indépendamment des discours des décideurs. Il est difficile d'évaluer l'interdisciplinarité exposée, aux corrosions du conformisme organisé.

\subsubsection{Une lueur d'espoir responsable}

«Tel est l'amateurisme de la prise de décision à la française qui fait que les mêmes erreurs sont constamment répétées » (Du Sautoy, 2020).

L'EIC (European Innovation Council) se dit être prêt à être patient et à accepter les échecs qui sont inhérents à la recherche technologique de pointe, en particulier dans le domaine des technologies high-tech issues de la science. Pour EU (2020), il ne s'agit pas de prendre des risques non calculés, voire «stupides », mais de prendre un risque d'investissement sur un thème considéré comme porteur que le marché seul n'accepterait pas. En particulier, ce soutien responsable serait pris dans les cas où les nouvelles technologies et les nouveaux modèles commerciaux seraient considérés comme disposant d'un potentiel à long terme d'apport d'avantages déterminants pour la société. Dans ce but, l'EIC doit rassembler des communautés scientifiques et d'entreprises qui sont aujourd'hui déconnectées, ou seulement liées de manière lâche ou informelle. Les financements de l'EIC seront dirigés vers la recherche interdisciplinaire de haute technologie et être centrés sur l'entreprise en mettant l'accent sur la simplicité et les besoins de l'innovateur. Dans le même temps, les procédures administratives de candidature, aujourd'hui longues et fastidieuses seront revues pour une évaluation qualitative agile, rapide, mais toutefois approfondie des candidats, de leurs idées et de leurs plans. Sur ces bases, les décisions de financement doivent être prises rapidement, en quelques semaines et non en quelques mois, voire plus...

\subsubsection{Un cadre du travail ambigu}

«Notre système d'éducation et notre environnement mercantile, deux environnements qui exècrent l'échec sont souvent fort peu propices à la créativité » (Du Sautoy, 2020).

D'aucuns peuvent écrire que les ressources sont suffisamment distribuées de manière à ce que les chercheurs puissent oublier leur arrimage à la société (cf. dans un cadre plus général, Bourlanges, 2017). Les débats actuels sur l'évolution continue vers plus de contrôle a priori peuvent ne pas soutenir la créativité ni le développement d'actions interdisciplinaires (MESRI, 2020). Mais, comme le souligne Pech (2017), de plus en plus de salariés, à la recherche d'un accomplissement personnel, cherchant plus d'autonomie, n'acceptent plus la subordination hiérarchique. Allaire et al (2016) 
donnent des chiffres sur cette évolution mesurable. «Certains s'enfuient vers l'entreprenariat, signe non pas d'une nouvelle valorisation de l'entreprise mais plutôt d'un discrédit croissant de la bureaucratie » (Pech, 2017) et, en plus, selon Colin (2015), cette dynamique se réalise dans le « chaudron de la révolution numérique ». Pour, ce même auteur : «Un hacker, dont les principales qualités sont la rébellion et la créativité, ne peut pas être formé par une institution comme l'Education Nationale, qui prétend certes former des citoyens à l'esprit critique, mais dont l'organisation et le fonctionnement tendent en même temps à façonner les travailleurs dociles et stéréotypés que réclamait l'économie de masse »... Les hackers ou les makers sont généralement des personnes passionnées par des problèmes à résoudre avec comme fondement philosophique le « faire » (Graham, 2015 ; Lallement, 2015 ; André, 2017). Le développement d'un artisanat intellectuel peut être possiblement favorable à la créativité, mais se posera la question essentielle du passage de l'idée à l'innovation en réunissant des acteurs qui ont besoin de se connaître pour coopérer...

\section{Vers une feuille de route en impression 4D}

«La première chose que le commun des mortels cherche à faire lorsqu'on lui présente un nouvel outil, c'est de l'utiliser comme le précédent » (Leblanc, 2017).

Nous avons tenté de collecter les questions posées le long de cet article pour essayer de définir des voies de ressourcement du domaine 4D afin qu'il satisfasse un certain nombre de critères applicatifs. Elles font l'objet du tableau 5. Cependant, s'il est clair que le domaine est complexe, que les recherches correspondent à des disruptions, il n'est pas sûr qu'il constitue un jour une technologie à fort impact économique. Pour autant, des applications rentables et d'utilité sociale devraient émerger dans cette technologie de niche, probablement non générique, s'appuyant sur des aspects scientifiques et techniques nécessitant approfondissement et sur le métissage disciplinaire permettant de satisfaire des besoins industriels. Dans ces conditions, les développements proposés serviraient, en cas de succès, d'une bonne illustration de l'exploration d'objets-frontières (méthodes, interdisciplinarité, prise de risque, relation science-société, etc.).

\begin{tabular}{|c|c|c|}
\hline Thème général & Axe d'action & Commentaires \\
\hline \multirow[t]{4}{*}{$\begin{array}{l}\text { Science et } \\
\text { technologie } \\
\text { «Homogène }\end{array}$} & Matériaux & $\begin{array}{l}\text { 1- Recherche sur des matériaux à fort coefficient de déformation } \\
\text { susceptibles d'être fabriqués en impression } 3 \mathrm{D} \text { (printabilité) } \\
\text { 2- Recherche sur des matériaux à « bonne » tenue mécanique } \\
\text { 3- Recherche sur des matériaux stimulables à temps de réponse } \\
\text { courts (typiquement, inférieurs à la seconde) }\end{array}$ \\
\hline & Impression 3D & Imprimantes multi-matériaux \\
\hline & Stimulation & $\begin{array}{l}\text { Méthodes de stimulation compatibles avec l'application : taille ou } \\
\text { encombrement, énergie consommée, etc. }\end{array}$ \\
\hline & Design & $\begin{array}{l}\text { La question de la modélisation des déformations d'un objet massif } \\
\text { est particulièrement complexe, avec des difficultés majeures de } \\
\text { traitement du problème inverse (téléologie) }\end{array}$ \\
\hline \multirow[t]{4}{*}{$\begin{array}{l}\text { Science et } \\
\text { technologie } \\
\text { « hétérogène » }\end{array}$} & Matériaux & $\begin{array}{l}\text { 1- Recherche sur des matériaux à fort coefficient de déformation } \\
\text { susceptibles d'être fabriqués (ou non) en impression 3D (printabilité) } \\
\text { 2- Pour des assemblages de multi-matériaux, nécessité de trouver } \\
\text { des couples qui adhérent avec des tenues au cisaillement et à la } \\
\text { traction acceptables } \\
\text { 3- Pour des assemblages de type origami ou multi-matériaux } \\
\text { disposer d'éléments actifs à temps de réponse courts }\end{array}$ \\
\hline & Fabrication & $\begin{array}{l}\text { Plutôt par impression 3D, mais aussi par d'autres voies à inventer, } \\
\text { par exemple robotisées }\end{array}$ \\
\hline & Stimulation & $\begin{array}{l}\text { Par dispositifs électriques ou pneumatiques de faible taille } \\
\text { permettant des déformations localisées }\end{array}$ \\
\hline & Design & $\begin{array}{l}\text { La question de la modélisation des déformations d'un objet réalisé } \\
\text { par association de filaments (méta-matériaux) ou d'éléments de } \\
\text { surface (origamis) est difficile, mais commence à être traitée, avec, }\end{array}$ \\
\hline
\end{tabular}




\begin{tabular}{|l|l|l|}
\hline & & $\begin{array}{l}\text { pour l'instant, des difficultés de traitement du problème inverse } \\
\text { (téléologie) }\end{array}$ \\
\hline $\begin{array}{l}\text { Organisation, } \\
\text { interdisciplinarité }\end{array}$ & Créativité & $\begin{array}{l}\text { Problème nécessaire à traiter en dehors de cette réflexion; approche } \\
\text { par la curiosité de ce qui mené dans des domaines proches }\end{array}$ \\
\hline & $\begin{array}{l}\text { Soutien à la prise de } \\
\text { risque }\end{array}$ & $\begin{array}{l}\text { A affirmer et à mettre en œuvre ; à traiter en dehors de cette } \\
\text { réflexion }\end{array}$ \\
\hline & Interdisciplinarité & Apprentissage de la gestion de projet en sciences « dures » \\
\hline
\end{tabular}

Tableau 5. Actions à mener pour le développement de l'impression 4D

D'ailleurs, certains travaux envisagent déjà l'utilisation de technologies de fabrication additive pour réaliser des systèmes avec des méta-matériaux (cf. par exemple, Rafiee, Farahani et Therriault, 2020 : Kanu et al, 2019 ; Schaffner et al, 2018). Des progrès récents en termes d'impression multimatériaux 3D et 4D devraient pouvoir être exploités pour étendre l'espace de conception au-delà des géométries désormais classiques de ces matériaux. Il est alors envisageable d'associer différents matériaux, diverses technologies de fabrication (dont additive) pour créer des objets actifs répondant mieux à des besoins applicatifs.

\section{Discussion conclusive}

«Que le nocturne débouche brutalement au grand jour, le fait surprend chaque fois. Il révèle pourtant une existence d'en dessous, une résistance interne jamais réduite. Cette force à l'affût s'insinue dans les tensions de la société qu'elle menace [...]. Elle brise les clôtures [...]; elle s'ouvre des chemins qui laisseront après son passage $[\ldots]$, un autre paysage et un ordre différent » (De Certeau, 2005).

De nombreux développements technologiques récents sont souvent décrits comme révolutionnaires; tel a été le cas, et cela reste encore vrai pour la fabrication additive classée généralement dans les 10 technologies du futur. La devise "Move Fast and Break things" (avancer rapidement et casser les choses), adoptée initialement par Facebook, symbolise la volonté, voire la détermination, des entreprises de la Silicon Valley à perturber ce qu'elles considèrent comme des normes sociales, des idées politiques et des modèles économiques dépassés, souvent avec une approche «mieux vaut demander pardon, que la permission » en matière de respect de la loi (Boucher et al, 2020). Cette volonté laissant une large place à la prise de risque technologique, pourtant essentielle, ne suffit pas à la promotion d'une technologie prometteuse comme l'impression 4D. Elle est nécessaire, mais pas suffisante parce qu'il faut aller au-delà des preuves de concept pour démontrer et créer de la désirabilité sociale.

Dans les années 1990, l'impression 3D était une activité encore modeste et seules quelques entreprises et personnes avaient accès aux premières machines. En dehors de résines acryliques déjà commercialisées, les matières nécessaires étaient souvent d'un coût élevé et la qualité des logiciels encore défaillante pour réaliser des pièces complexes. " Au fur et à mesure que ces matériaux comme les résines, les plastiques et les métaux en poudre - se sont répandus, l'impression 3D dans le secteur manufacturier a pu prendre son plein essor. Comme la technologie a dépassé le stade du prototypage et est entrée en production, les matériaux ont évolué en conséquence » (Nichols, 2020). Trente années au moins ont été nécessaires pour l'implantation acceptée de la fabrication additive. Si l'en était de même pour l'impression 4D, il faudrait attendre 2040-2050 pour une stabilisation et une inscription dans les dispositifs de fabrication de cette technologie émergente (ce qui met en défaut les prévisions introduites dans le $§ 1$ ). Or, tout au long de ce document, on a montré que la 4D reprend à son compte les difficultés d'implantation de la technologie qui l'a précédée en ajoutant des difficultés complémentaires. Alors, faudra-t-il plus de temps pour que le succès soit au rendezvous? 
Tout n'est sans doute pas pourri dans le royaume de la disruption, même si elle est profondément mise à l'écart par les disciplines et l'innovation incrémentale (le «système»). Ce qui manque probablement, c'est de faire apparaître une tectonique de temporalités entre des recherches de qualité, mais traditionnelles et des procédés créatifs plutôt immatériels (au départ) pour lesquels les phénomènes disruptifs risquent de créer (ou pas) des effets possiblement importants... pour autant qu'on les laisse éclore et les soutiennent (prise de risque). Les opérations de disruption restent plutôt proches de l'idée, de la combine, du bricolage un peu savant, avec des investissements intellectuels modestes (au départ), mais c'est à essayer. Pour aller un peu plus loin, il faudrait explorer la complexité et la convergence de disciplines pour l'action issue du prolongement de l'idée heureuse (ou considérée comme telle). Dans ce contexte, l'interdisciplinarité et la gestion de projets constituent des étapes incontournables.

Par conséquent, le moment est venu dès à présent d'encourager les innovateurs, les chercheurs, les entrepreneurs et les investisseurs visionnaires et capables de changer la donne, d'orienter les ressources vers des idées, des entreprises et des investissements à fort impact, et de permettre à nos industries et à nos nouvelles entreprises de prendre la tête des grandes opportunités d'innovation émanant des technologies de pointe dans les années à venir. Il serait temps de montrer aux jeunes innovateurs talentueux (chercheurs et entrepreneurs) que l'Europe (la France ?) est devenue le meilleur endroit pour développer leur potentiel et réaliser leurs rêves et leurs ambitions. Mais, pour soutenir cet objectif, il faudrait sans doute fabriquer avec quelques volontaires motivés une forme de « contre-culture officielle» offrant l'opportunité de disposer de «moments de vide », d'errance mentale indispensables à la créativité, à même d'assurer une reconquête d'un domaine pour le relancer (avec le risque de penser que cette remise en cause ne devienne pas la nouvelle modernité officielle). Ce peut être le cas de l'impression 4D, qui, pourtant n'en est qu'à ses débuts... S'il est présomptueux d'inclure l'impression 3D dans le concept d'industrie 4.0, la brique technologique 4D est encore largement immature. Lorsqu'on cherche à saisir la dimension fonctionnelle des chaînes de valeur, il s'agit de comprendre comment les firmes parviendront à gérer la fragmentation qu'elles ont construite : fragmentation spatiale, mais aussi fragmentation organisationnelle et technologique avec des niches très limitées... La généricité de l'impression 4D est à développer pour être intégrée un jour dans le concept 4.0 en produisant à l'unité (ou presque), en rompant avec la logique de la production de masse et avec des stockages légers (Chekurov et al, 2018).

"L'industrialisation de la science » entraîne une bureaucratisation des programmes, avec une faible confiance dans les proposants avec de nombreuses justifications a priori; elle constitue un facteur particulièrement limitant à la prise de risque... Il ne faut sans doute pas s'étonner que la production des idées par les chercheurs n'offre plus le retour sur investissement espéré (Bloom et al, 2020). Or, comme le rappelle l'European Commission (2020), s'il a fallu 75 ans environ pour que les citoyens s'approprient le téléphone, il n'a fallu qu'un mois pour que l'on dispose de 100 millions d'acheteur de «Pokémon Go»... C'est cette dynamique exacerbée qui doit être prise en considération pour éviter de rester sur le bord du chemin.

Mais, entre une forme de militantisme indispensable pour le changement, l'engagement vers la démonstration scientifique pour entraîner la conviction du besoin de changer de cap et le maintien d'un éveil critique (voire autocritique) dans ce nouveau à explorer, il y a besoin de sortir d'une contestation gagnante, d'éviter une forme de propagande prosélyte, visant la conversion officielle réductrice des tenants du paradigme précédent. Ce n'est pas une question d'humilité, mais de raison : on aura pu faire un petit pas dans la direction souhaitée, mais le but restera à atteindre. Le système d'évaluation par des pairs trop spécialisés peut modifier le processus de production de connaissances en méprisant des visions sortant du cadre reconnu alors que les tenants d'un paradigme continuent à nourrir des espoirs sous-entendant que le corps de doctrine est « presque à nos portes ». Cependant, comme le rappellent Callon et Lascoumes (2020), on continue à approfondir au même endroit, sans rupture. «Il n'y a pratiquement pas de réunions, où l'on envisage 
des projets sans but clairement fixé et sans que l'on soit certain d'avoir choisi le bon chemin, alors que cela devrait constituer des moments indispensables d'exploration de l'inconnu »...

La réalité de la science est qu'en absence d'une logique déterministe d'action en recherche, devenant alors juste engagée en amélioration incrémentale, il faut du temps pour explorer l'inconnu, pour tâtonner, pour déterminer ce qui fonctionne et ce qui ne fonctionne pas, pour se remettre en cause en visitant des domaines inconnus pour soi. La recherche de possibles solutions inventives et constructives peut en cas de succès être interprétée comme une volonté de liquidation et de destruction de ce qui avait été considéré comme un pilier robuste. «Les faux départs et les impasses apparents sont des éléments normaux d'un processus qui, lorsqu'il est mené honnêtement, conduit à des découvertes et à des innovations qui ont changé le monde » (Smith et Bryn Williams-Jones, 2020 ; Smith et al, 2020). S'il faut alors que des solidarités nouvelles se développent pour l'action, il faut éviter que des antagonismes se cristallisent et se renforcent, surtout parce que l'objectif à atteindre est encore lointain. "Les autistes ne trouvent plus leur place [...]. Place à l'écoute et à l'attention, à l'empathie, à l'envie et à la volonté d'aller vers l'autre, de coopérer avec lui »... (Vittori, 2020).

Mais pour que la science soit digne de confiance permettant d'aller vers l'objectif révélé par Tibbits (2013), il faut certes que les chercheurs fassent preuve d'un engagement éclairé et d'un comportement responsable et ouvert aux autres, mais également que les donneurs d'ordre soient à même d'accorder à ceux, empêcheurs de penser en rond, qui veulent sortir des chemins tracés par ceux qui ont acquis une certaine légitimité, un minimum de soutien. Mais c'est prendre des risques pour soi de tenter de souvenir des déviants qui peuvent échouer ! Alors !

Peut-être faut-il rappeler que la science ne repose pas uniquement sur une seule publication. Dans le domaine de l'impression 4D (et plus largement dans la plupart des domaines où la science a à jouer un rôle d'éclairage), les études doivent être nombreuses, et elles sont évaluées et critiquées par d'autres experts en la matière, mais non confinées au discours majoritaire. Au fil du temps, les résultats peuvent être validés ou invalidés, et on peut en tirer des conclusions, des ouvertures et de nouvelles connaissances. Cette situation, illustrée par cet article d'alerte sur une possible crise de limitation des promesses initiales en impression 4D peut offrir des conditions nouvelles pour l'action. En situation de stabilité, on accumule des résultats, les publications sont soumises à des dispositifs de régulation connus. Mais, quand ils ne sont plus fonctionnels, le dérèglement par la revisite divergente s'impose comme élément de perturbation d'une possible sortie des impasses. Ainsi, comme l'explique Edgar Morin (cité par Bouiss, 2020) : « la crise est tributaire de l'aléa : à certains de ses moments carrefours, il est possible à une minorité, à une action individuelle, de faire basculer le développement dans un sens parfois hautement improbable ».

« C'est un privilège inespéré de rencontrer un adulte capable d'une intranquilité enfantine. J'y vois un gage de curiosité, d'allant, de débord, d'audace » (Muller-Colard, 2017).

\section{Références}

- 4D_Additive (2020) « Module Advanced Lattice de 4D_Additive pour les composants légers, intègres et économes » Micado, 5, 34-35.

- Abtan A.A. (2019) "Design and Fabrication of Origami Elements for use in a Folding Robot Structure" PhD from the University of Leeds - UK - http://etheses.whiterose.ac.uk/26458/1/Akeel\%20Abtan\%20Thesis\%20Final\%20V.pdf

- Ackerman E. (2016) "Swarm of Origami Robots Can Self Assemble Out of a Single Sheet" http://spectrum.ieee.org/automaton/robotics/robotics-hardware/harvard-self-folding-origami-robots

- AFIS - Association Française pour l'Information Scientifique (2020) « La science peut-elle persévérer dans l'erreur? $» \underline{\text { https://www.pseudo-sciences.org/La-science-peut-elle-perseverer-dans-1-erreur }}$ 
- Aharoni H., Xia Y., Zhang X., Kamien R.D., Yang S. (2018) "Universal inverse design of surfaces with thin nematic elastomer sheets" Proceedings of the National Academy of Sciences U.S.A., 115, 7206.

- Ahmed A.A., Musbah A., Atiyah A. (2020) "4D Printing Technology: A Revolution across Manufacturing" International Journal of Mechanical and Industrial Technology, $\underline{7}, 45-51$.

- Akrich M., Callon M., Latour B. (1988) « A quoi tient le succès des innovations. », Gérer et Comprendre - Annales des Mines, $\underline{11}$, 4-17.

- Alain (1962) «Vigiles de l'esprit » Gallimard Ed. - Paris.

- Alhadeff-Jones M. (2008) "Three Generations of Complexity Theories: Nuances and Ambiguities" Educational Philosophy and Theory, $\underline{40}, 66-82$.

- Allaire T., Bureau J., Le Serviget A.J., Pech T. (2016) «Le marché du travail dans les grandes aires urbaines en $2015 »$ Jobijoba et Terra-Nova Ed. - Paris.

- Alter N. (2000) «L'innovation, croyances et pratiques » PUF Ed. - Paris - France.

- Ambrosi A., Webster R.D., Pumera M. (2020) "Electrochemically driven multi-material 3D-printing" Applied Materials Today, $\underline{18}, 100530$.

- Ancla C. (2010) « Micro-gels sensibles au glucose pour la délivrance d'insuline » Thèse de l'Université de Bordeaux - http://ori-oai.u-bordeaux1.fr/pdf/2010/ANCLA_CHRISTOPHE_2010.pdf

- Anderson C. (2008) “The Long Tail: Why the Future of Business is Selling Less of More” Hachette Ed. - Paris.

- Angelovska J., Mavrikiou P.M. (2013) "Can creative web survey questionnaire design improve the response quality? http://aias.s3.amazonaws.com/website/uploads/1456843118275AIASWP131-1.pdf

- André J.C. (2017) "From Additive Manufacturing to 3D/4D Printing - Volume 1: From the first concept to the present applications; Volume 2: Improvement of the present technologies and constraints; Volume 3: Breakdown innovations: Programmable matter; 4D Printing and Bio-Printing” ISTE/Wiley Ed. - Londres - UK.

- André J.C. (2020 a) «Impression 3D : niches applicatives porteuses » Techniques de l'Ingénieur, Réf. BM 7970.

- André J.C. (2020 b) «L'émergence silencieuse de la complexité en impression 3D » Entropie, 20-1, 34 pp https://www.openscience.fr/L-emergence-silencieuse-de-la-complexite-en-impression-3D

- André J.C. (2020) « Recyclage des déchets d'impression 3D » Techniques de l’Ingénieur - Réf. BM 7975.

- André J.C., Le Mehauté A., De Witte O. (1984) « Dispositif pour réaliser un modèle de pièce industrielle » Brevet français $n^{\circ} 8411241,16.07 .1984$.

- André J.C., Masse R. (2002) «L'expertise, la science et l'incertitude : l'expertise scientifique ou la langue d'Ésope » Environnement Risques et Santé 1, 299-306.

- Araujo-Jorge M.M. (2001) «L'impact épistémologique de la complexité » in M. Crozon, Y. Jacquin Ed. « L'élémentaire et le complexe » EDP Sciences Ed. - Paris.

- Atlan H. (1999) « Les étincelles du hasard » Seuil Ed. - Paris.

- Atlantico (2020) «Vers une pénurie d'idées nouvelles? L'étude qui montre que l'innovation véritable progresse peu malgré un nombre de chercheurs dans le monde toujours grandissant» https://www.atlantico.fr/decryptage/3589287/vers-une-penurie-d-idees-nouvelles--1-etude-qui-montre-que-1innovation-veritable-progresse-peu-malgre-un-nombre-de-chercheurs-dans-le-monde-toujours-grandissant-yvesmichaud-

- Avenier M.J. (2019) «Les Sciences de l'artificiel : une conceptualisation révolutionnaire de sciences fondamentales à parachever » Projectics / Proyéctica / Projectique, 24, 43-56.

- Bachelard G. (2003) «Le Nouvel Esprit Scientifique » Presses Universitaires de France Ed. - Paris.

- Bakar M., Djaider F. (2007) "Effect of Plasticizers Content on the Mechanical Properties of unsaturated Polyester Resin" Journal of Thermoplastic Material, 20, 53-64.

- Bar-Cohen Y. (2004) "Electroactive Polymer (EAP) Actuators as Artificial Muscles: Reality, Potential, and Challenges" SPIE Ed. -Bellingham - USA.

- Barbieri-Masini E. (2000) «Penser le Futur : L'essentiel de la prospective et de ses méthodes » Dunod Ed. - Paris France. 
- Barnard M. (2017) "7 Reasons: The Future is Electric" https://cleantechnica.com/2017/11/12/7-reasons-futureelectric/?utm_source=feedburner\&utm_medium=feed\&utm_campaign=Feed\%3A+IM-

cleantechnica+\%28CleanTechnica\%29

- Battu D. (2018) «L'histoire et l'économie du monde accompagnées par les TIC » ISTE Ed. - Londres - UK.

- Beguin P., Cerf M. (2009) «Dynamique des savoirs, dynamique des changements » Octarès Ed. - Toulouse.

- Beltagui A., Sesis A., Stylos N. (2019) "3D Printing, Maker-spaces and Innovation: A Bricolage Perspective" International Product Development Management Conference - Leicester - UK https://www.researchgate.net/publication/333662364_3D_Printing_Makerspaces_and_innovation_a_bricolage_per spective

- Berchon M. (2020) «Le grand livre de l'impression 3D » Eyrolles Ed. - Paris.

- Blanc J.V. (2019) « Quels sont les liens entre souffrance psychique et créativité ? 》 https://www.psychologies.com/Moi/Problemes-psy/Troubles-Maladies-psy/Articles-et-Dossiers/Quels-sont-lesliens-entre-souffrance-psychique-et-creativite

- Blanchet M. (2016) «Industrie 4.0 : nouvelle donne industrielle, nouveau modèle économique » Géo-économie, $\underline{82}$, 37-53.

- Bloom N., Jones C., Van Reenen J., Webb M. (2020) "Are Ideas Getting Harder to Find?" American Economic Review, 110, 1104-44.

- Bœuf G. (2014) « Bio-mimétisme et bio-inspiration » Vraiment durable, 5/6, 43-55.

- Bonaccorsi A. (2020) "Two Decades of Experience in Research Assessment in Italy" Scholarly Assessment Reports, 2, 16 pp - http://doi.org/10.29024/sar.27

- Boucher P., Bentzen N., Lațici T., Madiega T., Schmertzing L., Szczepański M. (2020) "Disruption by technology Impacts on politics, economics and society" European Parliamentary Research Service https://www.europarl.europa.eu/RegData/etudes/IDAN/2020/652079/EPRS IDA(2020)652079 EN.pdf

- Bouiss O. (2020) « Qu'est-ce qu'une « crise »? " https://theconversation.com/quest-ce-quune-crise136026? utm medium=email\&utm campaign=La\%20lettre\%20de\%20The\%20Conversation \%20France\%20du\%20 20\%20mai\%202020\%20-

\%201627315626\&utm_content=La\%20lettre\%20de\%20The\%20Conversation\%20France\%20du\%2020\%20mai\%2 $\underline{02020 \% 20-}$

\%201627315626+CID_e7d11b53fbb6efa351d34998bf4c418c\&utm_source=campaign_monitor_fr\&utm_term=Qu est-ce\%20quune \%20\%20crise

- Bounouira F. (2015) "Les gels, aspects théoriques et applications » Thèse de pharmacie - Université Mohammed V Rabat - Maroc - $\quad$ http://ao.um5.ac.ma/xmlui/bitstream/handle/123456789/14759/P\%2007-

2015.pdf? sequence $=1 \&$ is Allowed $=\mathrm{y}$

- Bourcier D., Van Andel P. (2017) « C’est quoi la sérendipité ? » Le courrier du livre Ed. - Paris.

- Bourlanges J.L. (2017) cité par Peach T. «Insoumissions - Portrait de la France qui vient » Seuil Ed. - Paris.

- Boyer F., Mauny J., Porez M. (2019) «Dynamique de la locomotion bio-inspirée en robotique » Techniques de l’Ingénieur, S7757 V1, 22pp.

- Boydston A.J., Cao B., Nelson A., Ono R.J., Saha A., Schwartz J.J., Thrasher C.J. (2018) “Additive manufacturing with stimuli-responsive materials" Journal of Material Chemistry, A6, 20621-20645.

- Buriak I.A., Zhurba V.O., Vorobjov G.S., Kulizhko V.R., Kononov O.K., Rybalko O. (2016) "Metamaterials: Theory, Classification and Application Strategies (Review)" Journal of Nano- and Electronic Physics, $\underline{8}, 04088$.

- Callens S.J.P., Tümer N., Zadpoor AA. (2019) "Hyperbolic origami-inspired folding of triply periodic minimal surface structures" Applied Material Today, 15, 453-461.

- Callon M., Lascoumes P. (2020) « Penser l'après : Le Covid-19 pousse les scientifiques hors de leurs laboratoires » https://theconversation.com/penser-lapres-le-covid-19-pousse-les-scientifiques-hors-de-leurs-laboratoires-

137336 ?utm medium=email\&utm campaign=La\%20lettre\%20du\%20week-

end $\% 20 \mathrm{de} \% 20$ The $\% 20$ Conversation $\% 20$ France $\% 20$ -

$\% 201629615653 \& u t m \_c o n t e n t=L a \% 201$ ettre\%20du\%20week-

end $\% 20 \mathrm{de} \% 20$ The $\% 20$ Conversation\%20France $\% 20$ -

\%201629615653+CID_33de43f7fe3fd077fee1d632d98d45e8\&utm_source=campaign_monitor_fr\&utm_term=Pen

ser\%20laprs\%20\%20Le\%20Covid-19\%20pousse\%20les\%20scientifiques\%20hors\%20de\%20leurs\%20laboratoires

(C) 2021 ISTE OpenScience - Published by ISTE Ltd. London, UK - openscience.fr

Page | 38 
- Celhay F., Cusin J. (2011) « Comment innover dans une organisation prisonnière de la tradition et de son succès et faisant face à un environnement réfractaire à la nouveauté ? Le cas des vins de Bordeaux » Gestion, $\underline{36}, 44-53$.

- Chalmers A. (1990) "Science and its fabrication" Open University Press Ed. - Buckingham - UK.

- Chan H.K, Griffin J., Lim J.L., Zeng F., Chiu A. (2018) "The impact of 3D printing technology on the supply chain: Manufacturing and legal perspectives" International Journal of Production Economics, 205, 156-162.

- Chekurov S., Metsä-Kortelainen S., Salmi M., Roda I., Jussila A. (2018) "The perceived value of additively manufactured digital spare parts in industry: An empirical investigation" International Journal of Production Economics, 205, 87-97.

- Chen T., Bilal O.R., Lang R., Daraio C., Shea K. (2019) “Autonomous deployment of a solar panel using elastic origami and distributed shape-memory-polymer actuators" Physical Review Applied, 11, 064069.

- Cheng F., Zhang Y., Yin R., Yu Y. (2010) "Visible light induced bending and unbending behavior of crosslinked liquid-crystalline polymer films containing azotolane moieties” Journal of Material Chemistry, 20, 4888-4896.

- CNRS (2002) «Le conseil scientifique du CNRS - Journées thématiques \& recommandations » http://www.cnrs.fr/comitenational/doc/archives/bilan/bilan cs20012005.pdf

- Colin N. (2015) «La richesse des nations après la révolution numérique » Terra-Nova Ed. - Paris.

- Colosimo J.F. (2018) «Aveuglements - Religions, guerres, civilisations » Ed. du Cerf - Paris.

- Constant B. (2010) «De la liberté des anciens comparée à celle des modernes » Mille et une nuits Ed. - Paris.

- Corazza G. (2015) "Potential originality and effectiveness: The dynamic definition of creativity" Creativity Research Journal, 28, 258- 267.

- Coulais C., Teomy E., de Reus K., Shokef Y., van Hecke M. (2016) "Combinatorial design of textured mechanical metamaterials" Nature, $\underline{\mathrm{S} 35}$, 529-535.

- Cropley D.H. (2015) «Creativity in Engineering - Novel Solutions to Complex Problems » Academic Press Ed. Londres - UK.

- Crozier M., Tillette B. (2000) «Quand la France s'ouvrira » Fayard Ed. - Paris.

- Cui T.J., Qi M.Q., Wan X., Zhao J., Cheng Q. (2014) "Coding metamaterials, digital metamaterials and programmable metamaterials" Light: Science \& Applications, $\underline{3}$, e218.

- Cui T.J., Smith D., Liu R. (2010) "Metamaterials - Theory, Design, and Applications" Springer Verlag Ed. - NewYork - USA.

- Culot G., Orzes G., Sartor M., Nassimbenia G. (2020) "The future of manufacturing: A Delphi-based scenario analysis on Industry 4.0” Technology Forecast \& Social Change, 157, 120092.

- Dachs B., Palcic I. (2020) "Not for everyone? Product characteristics and digital production technologies in manufacturing" Revue d'économie industrielle, 169, 37-56.

- Danzo A., André J.C. (2020) “« Que peut apporter une modélisation mathématique à la maitrise du bio-printing ? » Entropie, 20-1, 32pp - http://openscience.fr/Que-peut-apporter-une-modelisation-mathematique-a-la-maitrise-dubio-printing

- De Certeau M. (2005) «La possession de Loudun» Gallimard Ed. - Paris.

- De Simone A., Gidoni P., Noselli G. (2015) «Liquid crystal elastomer strips as soft crawlers » Journal of Mechanics \& Physics of. Solids, $\underline{84}, 254-272$.

- Dean T., Zhang H., \& Xiao Y. (2020) "The role of complexity in the Valley of Death and radical innovation performance" Technovation - https://doi.org/10.1016/j.technovation.2020.102160

- Descartes R. (1990) « Discours de la méthode » Nathan Ed. - Paris.

- Dedehayir O., Mäkinen S.J., Ortt J.R. (2018) "Roles during Innovation Ecosystem Genesis: A Literature Review" Technological Forecasting and Social Change, 136, 18-29.

- Dillaerts H. (2014) «Le libre accès et le financement de projets de recherche transversaux : des vecteurs d'interdisciplinarité dans l'économie de la connaissance ? » Revue européenne des sciences sociales, 52, 111-135.

- Dimassi S., Demoly F., Cruz C., Qi H.J., Kim K.Y., André J.C., Gomes S. (2020) “An ontology-based framework to formalize and represent 4D printing knowledge in design" Computers in Industry, sous presse. 
- Ding Z., Weeger O., Qi H.J., Dunn M.L. (2018) "4D rods: 3D structures via programmable 1D composite rods" Materials \& Design, 137, 256-265.

- Donnadieu G., Karsky M. (2002) «La systémique ; penser et agir dans la complexité » Ed. Liaisons - Paris.

- Dorow P., Davila G., Varvakis G., Vallejos R. (2015) “Generation of Ideas, Ideation and Idea Management" Navus, $\underline{5}, 51-59$.

- Dosi G. (1988) “Sources, Procedures, and Microeconomic Effects of Innovation” Journal of Economic Literature, 26, 1120-1171.

- Du F.P., Ye E.Z., Yang W., Shen T.H., Tang C.Y., Xie X.L., Zhou X.P., Law W.C. (2015) "Electroactive shape memory polymer based on optimized multi-walled carbon nanotubes/polyvinyl alcohol nanocomposites" Composites - Part B, $\underline{68}, 170-175$.

- Du Sautoy M. (2020) "Le code de la créativité - Comment l'IA apprend à écrire, peindre et penser » Ed. Héloïse d'Ormesson - Paris.

- Duduta M., Hajiesmaili E., Zhao H., Wood R.J., Clarke D.R. (2019) "Realizing the potential of dielectric elastomer artificial muscles" Proceedings of the National Academy of Science of the USA, 116, 2476-2481.

- Dufaud O., Marchal P., Corbel S. (2002) "Rheological properties of PZT suspensions for stereo-lithography" Journal of the European Ceramic Society, 22, 2081-2092.

- Dumont N.T. (2018) «Les heuristiques fonctionnelles » https://www.westattitude.com/single-post/2018/04/25/LESHEURISTIQUES-fonctionnelles

- Durach C.F., Kurpjuweit S., Wagner S.M. (2017) "The impact of additive manufacturing on supply chains" International Journal of Physical Distribution \& Logistics Management, 47, 954-971.

- Ercole F., Davis T.P., Evans R.A. (2010) "Photo-responsive systems and biomaterials: photochromic polymers, lighttriggered self-assembly, surface modification, fluorescence modulation and beyond" Polymer Chemistry, 1, 37-54.

- EU (2020) "A vision for the European Innovation Council: from deep-tech research to visionary innovation and scale-ups" https://ec.europa.eu/research/eic/pdf/ec rtd eic-vision.pdf

- European Commission (2020) "Science, Research and Innovation Performance of the EU 2020 - A fair, green and digital Europe" https://ec.europa.eu/info/sites/info/files/srip/2020/ec rtd srip-2020-report.pdf

- Evans J.D., Bon V., Senkovska I., Lee H.C., Kaskel S. (2020) "Four-dimensional metal-organic frameworks" Nature Communications, 11, 2690.

- Fang L., Chen S., Fang T., Fang J., Lu C., Xu Z. (2017) "Shape-memory polymer composites selectively triggered by near-infrared light of two certain wavelengths and their applications at macro-/microscale" Composite Science Technology, 138, 106-116.

- Fillingim K.B., Nwaeri R.O., Paredis C.J., Rosen D., Fu K. (2020) "Examining the effect of design for additive manufacturing rule presentation on part redesign quality" Journal of Engineering Design, 31, 427-460.

- Fressoz J.B. (2018) "Quand la catastrophe suit son cours" 63-75 in Birnbaum J. Ed. « De quoi avons-nous peur?» Gallimard Ed. - Paris.

- Friedman G. (1952) "Les conséquences sociales du progrès technique" UNESCO - Bulletin International des Sciences Sociales, $\underline{4}, 251-272$.

- Frigant V. (2020) «L'industrie 4.0, vers une dé-globalisation des chaînes de valeur ? Effets attendus de la robotique industrielle avancée et de la fabrication additive sur le système de coordination » Revue d'économie industrielle, $\underline{169}, 127-160$.

Future Bridge (2020) "4D Printing - The Technology of the Future" https://www.futurebridge.com/industry/perspectives-mobility/4d-printing-the-technology-of-the-future/

- Gao B., Yang Q., Zhao X., Jin G., Ma Y., Xu F. (2016) “4D Bio-printing for Biomedical Applications” Trends in Biotechnology, $\underline{34}, 746-756$.

- Gao W., Zhang Y., Ramanujan D., Ramani K., Chen Y., Williams C.B., Wange C.C.L., Shin Y.C., Zhang S., Zavattieri P.D. (2015) "The status, challenges, and future of additive manufacturing in engineering" ComputerAided Design, 69, 65-89.

- Garcia I. (2020) “4D printing: An overview into a disruptive technology" https://chemindustryrevolution.com/4dprinting-an-overview-into-a-disruptive-technology/ 
- Ge Q., Qi J., Dunn M.L. (2013) “Active materials by four dimensions printing” Applied Physical Letters, $\underline{103}$, 131901.

- Ge Q., Dunn C.K., Qi H.J., Dunn M.L. (2014) “Active origami by 4D printing” Smart Materials and Structures, $\underline{23}$, 094007.

- Ge Q., Sakhaei A.H., Lee H., Dunn C.K., Fang N.X., Dunn M.L. (2016) "Multi-material 4D Printing with Tailorable Shape Memory Polymers" Scientific Reports $\underline{6}, 31110$.

- Gide A. (2008) « les faux-monnayeurs » Folio Ed. - Paris.

- Godina R., Ribeiro I., Matos F., Ferreira B.T., Carvalho H., Peças P. (2020) "Impact Assessment of Additive Manufacturing on Sustainable Business Models in Industry 4.0 Context” Sustainability, 12, 7066.

- Gong X., Yang K., Xie J., Wang Y., Kulkarni P., Hobbs A., Mazzeo A. (2016) "Rotary Actuators Based on Pneumatically Driven Elastomeric Structures" Advanced Materials, 28, 7533-7538.

- Goo B., Hong C.H., Park K. (2020) "4D printing using anisotropic thermal deformation of 3D-printed thermoplastic parts" Materials \& Design, $\underline{188}, 108485$.

- Goria S. (2019) "How the interpretation frame inventory method can help to identify some of the rigidities of an innovation system” Journal of Innovation Economics \& Management, 28, 29-51.

- Goria S., Levassor C. (2015) «L'emploi des cartes cognitives pour la formation à la maitrise de l'information ». M. Ihadjadène, A. Saemmer, C. Baltz Ed. "Culture informationnelle : vers une propédeutique du numérique » Hermann Ed. - Paris.

- Graham P. (2015) “Hackers and painters - Big ideas from computer age” O’Reilly Media Ed. - Sebastopol - USA.

- Grosul M., Feist G.J. (2014) "The Creative Person in Science" Psychology of Aesthetics, Creativity, and the Arts, American Psychological Association, $\underline{8}, 30-43$.

- Hacking I. (2001) «Entre science et réalité. La construction sociale de quoi? » La Découverte Ed. - Paris.

- Hager M., Bode S., Weber C., Schubert U. (2015) "Shape memory polymers: Past, present and future developments" Progress in Polymer Science, $\underline{49}, 3-33$.

- Hahn V., Kiefer P., Frenzel T., Qu J., Blasco E., Barner-Kowollik C., Wegener M. (2020) "Rapid Assembly of Small Materials Building Blocks (Voxels) into Large Functional 3D Metamaterials" Advances in Functional Materials, 30, 1907795.

Hally A. (2020) " La hype autour de l'hyperpersonnalisation est finie 》 https://www.strategies.fr/actualites/medias/4053436W/-la-hype-autour-de-l-hyperpersonnalisation-est-finieandrew-hally-bynder-.html

- Han X.J., Dong Z.Q., Fan M.M., Liu Y., Li J.H., Wang Y.F., Yuan Q.J. (2012) "pH-induced shape-memory polymers" Macromolecules Rapid Communications, $\underline{33}$, 1055-1060.

- Hannibal M., Knight G. (2018) "Additive manufacturing and the global factory: disruptive technologies and the location of international business" International Business Review, 27, 1116-1127.

- Hansson S.O. (2010) «L'incertitude en matière de technologie » Annales des Mines - Responsabilité et environnement, $\underline{57}, 70-74$.

- Hegel G. (2003) «La raison dans l'histoire » Poche 10/18 Ed. - Paris.

- Heemsbergen L., Daly A., Lu J., Birtchnell T. (2019) "3D-printed Futures of Manufacturing, Social Change and Technological Innovation in China and Singapore: The Ghost of a Massless Future?" Science, Technology and Society, 24, 254-270.

- Hirsch M., Charlet A., Amstad E. (2020) "3D Printing of Strong and Tough Double Network Granular Hydrogels" Advanced Functional Materials, 2005929 - https://onlinelibrary.wiley.com/doi/full/10.1002/adfm.202005929

- Hisour (2020) « Polymères électro-actifs » https://www.hisour.com/fr/electroactive-polymers-42852/

- Hobbes T. (1993) «Le citoyen et les fondements de la politique » Flammarion Ed. - Paris.

- Holm P., Goodsite M.E., Cloetingh S., Agnoletti M., Moldan B., Lang D., Leemans R., Moeller J.O., Buendia M.P., Pohl W., Scholz R.W., Sors A., Vanheusden B., Yusoff K., Zondervan R. (2013) "Collaboration between the natural, social and human sciences in Global Change Research" Environmental Science and Policy, 28, 25-35. 
- Hu J., Zhu Y., Huang H., Lu J., (2012) "Recent advances in shape memory polymers: Structure, mechanism, functionality, modeling and applications" Progress in Polymer Science, 37, 1720-1763.

- Hu F., Wang W., Cheng J., Bao Y. (2020) "Origami spring-inspired metamaterials and robots: An attempt at fully programmable robotics" Science Progress, 103, 1-19.

- Hwang Y., Paydar O.H., Candler R.N. (2015) "Pneumatic micro-finger with balloon fins for linear motion using 3D printed molds" Sensors \& Actuators, 234, 65-71.

- Ilic-Stojanovic S., Nikolic L., Nikolic V., Petronic S., Stankovic M., Mladenovic-Ranisavljevic I. (2011) "Stimulisensitive hydrogels for pharmaceutical and medical applications" Facta Universitatis, $\underline{9}$, 37-56.

- Ion A., Frohnhofen J., Wall L., Kovacs R., Alistar M., Lindsay J., Lopes P., Chen H.T., Baudisch P. (2016) "Metamaterial Mechanisms" Proceedings of the UIST'16 https://hpi.de/fileadmin/user upload/fachgebiete/baudisch/projects/metamaterial/metamaterialmechanisms/2016UIST_Metamaterial_Mechanisms_authors_copy_.pdf

- Ion A., Kovacs R., Schneider O.S., Lopes P., Baudisch P. (2018) "Metamaterial Textures" https://www.researchgate.net/profile/Alexandra_Ion/publication/324669839_Metamaterial_Textures/links/5af18ee daca272bf4255bef2/Metamaterial-Textures.pdf

- Ion A., Wall L., Kovacs R., Baudisch P. (2018 a) "Digital Mechanical Metamaterials" https://www.researchgate.net/profile/Alexandra_Ion/publication/314263173_Digital_Mechanical_Metamaterials/li nks/5a0313b3a6fdcc6b7c9c2e1b/Digital-Mechanical-Metamaterials.pdf

- Janbaz S., Bobbert F.S.L., Mirzaali M.J., Zadpoora A.A. (2019) "Ultra-programmable buckling-driven soft cellular mechanisms" Material Horizons https://pubs.rsc.org/en/content/articlelanding/2019/MH/c9mh00125e\#!divAbstract

- Jardim-Goncalves R., Romero D., Grilo A. (2017) "Factories of the Future: Challenges and Leading Innovations in Intelligent Manufacturing” International Journal of Computer Integrated Manufacturing, 30, 4-14.

- Jayashankar D.K., Gupta S.S., Stella L.Y.N., Tracy K. (2019) "3D printing of compliant passively actuated 4d structures" Solid Freeform Fabrication 2019: Proceedings of the 30th Annual International Solid Freeform Fabrication Symposium - An Additive Manufacturing Conference Reviewed Paper" http://utw10945.utweb.utexas.edu/sites/default/files/2019/082\%203D\%20Printing\%20of\%20Compliant\%20Passiv ely\%20Actuated\%204D\%20Str.pdf

- Jehn K., Northcraft G., Neale M. (1999) "Why Differences Make a Difference: A Field Study of Diversity, Conflict, and Performance in Workgroups" Administrative Science Quarterly, 44, 741-763.

- Jeong H.Y., An S.C., Seo I.C., Lee E., Ha S., Kim N., Jun Y.C. (2019) "3D printing of twisting and rotational bistable structures with tuning elements" Scientific Reports, $\underline{9}, 324$.

- Jian B. (2020) «Conception basée sur les origamis pour l'impression 4D de structures déployables » Thèse de l'UTBM - Sévenans.

- Jian B., Demoly F., Zhang Y., Gomes S. (2019) “An origami-based design approach to self-reconfigurable structures

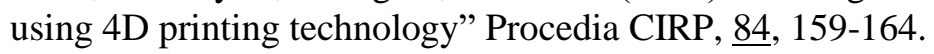

- Jiang H., Wang Z., Jin Y., Chen X., Li P., Gan Y., Lin S., Chen X. (2020) "Design, Control, and Applications of a Soft Robotic Arm" https://arxiv.org/pdf/2007.04047.pdf

- Jin B., Song H., Jiang R., Song J., Zhao Q., Xie T. (2018) "Programming a crystalline shape memory polymer network with thermo- and photo-reversible bonds toward a single-component soft robot" Science Advances, $\underline{4}$, eaao3865.

- Joseph E., Holm V. (2015) "Makerspaces and Contributions to Entrepreneurship" Procedia Social Behavior Science, $195,24-31$.

- Kadic M., Milton G.W., van Hecke M., Wegener M. (2019) “3D metamaterials” Nature Reviews -Physics, 1 , 198210.

- Kagermann H., Wahlster W., Helbig J. (2013) "Umsetzungsempfehlungen für das Zukunftsprojekt Industrie 4.0. Abschlussbericht des Arbeitskreises Industrie 4.0. ACATECH" Deutsche Akademie der Technikwissenschaften in Berlin - Allemagne.

- Kanu N.J., Gupta E., Vates U.K. Singh G.K. (2019) “ An insight into biomimetic 4D printing” Royal Society of Chemistry Advances, $\underline{9}, 38209$. 
- Khim T., Zhu L., Al-Kaysi R.O., Bardeen C.J. (2014) "Organic photomechanical material” ChemPhysChem, $\underline{15}$, 400-414.

- Khoo Z.X., Mei Teoh J.E., Liu Y., Chua C.K., Yang S., An J., Leonga K.F., Yeong W.Y. (2015) "3D printing of smart materials: A review on recent progresses in 4D printing" Virtual and Physical Prototyping, 10, 103-122.

- Krödel S., Li L., Constantinescu A., Daraio C. (2017) "Stress relaxation in polymeric micro-lattice materials" Materials and Design, $\underline{130}, 433-441$.

- Kuksenok O., Balazs A.C. (2013) "Modeling the photo-induced reconfiguration and directed motion of polymer gels" Advanced Functional Materials, 23, 4601-4610.

- Kunovjanek M., Reiner G. (2020) "How will the diffusion of additive manufacturing impact the raw material supply chain process?” International Journal of Production Research, ㅌ, 1540-1554.

- Lallement M. (2015) « L’âge du faire - Hacking, travail, anarchie » Seuil Ed. - Paris.

- Lam H.K.S., Ding L., Cheng T.C.E., Zhou H. (2019) “The impact of 3D printing on stock returns: a contingent dynamic capabilities perspective" International Journal of Operations and Production Management, 39, 935-961.

- Lau H.F. (2019) "3D-Printed Inflatable Actuators - Design and Development of Soft Actuators for a PneumaticallyActuated Soft Robotic Arm” https://www.research-collection.ethz.ch/handle/20.500.11850/357220

- Le Duigou A., Beaugrand J., Castro M. (2017) «Compréhension des mécanismes d'actionneur des pommes de pin pour améliorer les performances des bio-composites hygro-morphes » Journées Nationales sur les Composites 2017, Ecole des Ponts, ParisTech (ENPC) - https://hal.archives-ouvertes.fr/hal-01623672/document

- Le Duigou A., Chabaud G., Scarpa F., Castro M. (2019) "Bioinspired Electro-Thermo-Hygro Reversible ShapeChanging Materials by 4D Printing” Advanced Functional Materials, 2019, 1903280.

- Le Digou A., Castro M. (2020) «La pomme de pin, une force de la nature !» https://irdl.fr/la-pomme-de-pin-uneforce-de-la-nature/

- Le Moigne J.L. (2001) «Interdisciplinarité ; comprendre pour faire ? » La lettre chemin faisant, 39. http://www.intelligence-complexite.org/fileadmin/docs/39.pdf

- Leblanc D. (2017) «Smart world - Comment de simples idées deviennent de grandes inventions » Edilivres Ed. Saint Denis.

- Leleu-Merviel S., Boulekbache H. (2014) «Recherches en design - Processus de conception, écriture et représentations » ISTE Ed. - Londres - UK.

- Lendlein A. (2010) "Shape-Memory Polymers" Springer Ed. - Berlin/Heidelberg - Allemagne.

- Lendlein A., Gould O. (2019) "Reprogrammable recovery and actuation behavior of shape-memory polymers" Nature Review Materials, $\underline{4}, 116-133$.

- Lévi-Strauss (1990) «La pensée sauvage » Pocket Ed. - Paris.

- Levinas E. (1990) « Totalité et infini : essai sur l'extériorité » Poche Ed. - Paris.

- Lévy-Leblond J.M. (2020) « Le tube à essais - Effervesciences » Seuil Ed. - Paris.

- Li S., Stampfl J., Xu H., Xu E., Diaz E., Malkin V., Rus D., Wood R. (2019) “A Vacuum-driven Origami “Magicball” Soft Gripper" IEEE - https://dspace.mit.edu/handle/1721.1/120930

- Liu Y., Xu B., Sun S., Wei J., Wu L., Yu Y. (2017) "Humidity- and photo-induced mechanical actuation of crosslinked liquid crystal polymers" Advanced Materials, 29, 1604792.

- Lizarralde I., Larrasquet J.M., Coutts N. (2011) "Design and innovation in the face of complexity (Towards new challenges of linking systems and learning)" Projectics/Proyéctica/Projectique, $\underline{8-9}, 199-211$.

- Lohmuller P., Favre J., Kenzari S., Piotrowski B., Peltier L., Laheurte P. (2019) "Architectural effect on 3D elastic properties and anisotropy of cubic lattice structures" Materials \& Design, 182, 108059.

- Lovric D., Schneider G. (2020) "What Kind of Chief Innovation Officer Does Your Company Need?" https://hbr.org/2019/11/what-kind-of-chief-innovation-officer-does-your-company-need

- Ma J., Franco B., Tapia G., Karayagiz K., Johnson L., Liu J., Arroyave R., Karaman I., Elwany A. (2017) "Spatial control of functional response in 4D-printed active metallic structures" Scientific Reports, $\underline{7}, 46707$.

- Mac Causland T. (2020) “3D Printing’s Time to Shine” Research-Technology Management, 63, 62-65. 
- Mahimwalla Z. (2013) "Characterization of photo-induced photo-mechanical responses in azo-benzene polymers" PhD Université McGill - Montréal - Canada.

- Mahimwalla Z., Yager K., Mamiya J.I., Shishido A., Priimagi A., Barrett C. (2012) “Azo-benzene photo-mechanics prospects and potential applications" Polymer Bulletin, $\underline{69}$, 967-1006.

- Mamasioulas A., Mourtzis D., Chryssolouris G. (2020) "A manufacturing innovation overview: concepts, models and metrics" International Journal of Computer Integrated Manufacturing, $\underline{33}$, 769-791.

- Marsilli O. (2000) "Technological regimes and sources of entrepreneurship" - Working Paper 00.10 http://citeseerx.ist.psu.edu/viewdoc/download?doi=10.1.1.959.5438\&rep=rep1\&type=pdf

- Maslow A. (1966) “The Psychology of Science” Harper and Row Ed. - New-York - USA.

- Market Analysis Report (2017) "4D Printing Market Size, Share \& Trends Analysis Report by Material (Programmable Carbon Fiber, Programmable Wood, Programmable Textiles), By End-Use, by Region, and Segment Forecasts, 2018 - 2025" - https://www.grandviewresearch.com/industry-analysis/4d-printing-market

- Market and Market (2020) “4D Printing Market by Material (Programmable Carbon Fiber, Programmable Wood Custom Printed Wood Grain, Programmable Textiles), End User (Aerospace, Automotive, Clothing, Construction, Defense, Healthcare \& Utility) \& Geography - Global Trends \& Forecasts to 2019 - 2025" https://www.marketsandmarkets.com/Market-Reports/4d-printing-market-3084180.html

- Market Intellica (2019) “4D Printing Market Research: Global Status \&Forecast by Geography, Type \& Application $(2015,2025) "$ https://www.marketintellica.com/report/MI74639-4d-printing-market-research-global-status

- Market Screener (2020) "The Global 4D Printing Market to Garner \$236.22 Billion by the year 2028" https://www.marketscreener.com/quote/stock/AUTODESK-INC-40246776/news/The-Global-4D-Printing-Marketto-Garner-236-22-Billion-by-the-year-2028-31104240/

- Market Watch (2020) "4D Printing Market Size, Growth Research Analysis and Share to attain over US \$419.5 million by 2026 - New Report" https://www.marketwatch.com/press-release/4d-printing-market-size-growthresearch-analysis-and-share-to-attain-over-us-4195-million-by-2026---new-report-2020-09-

$\underline{02 ? \bmod =\mathrm{mw} \_q u o t e \_n e w s}$

- Marsot J. (2002) “Conception et ergonomie - Méthodes et outils pour intégrer l'ergonomie dans le cycle de conception des outils à mains » Note Scientifique et Technique 219 de l'INRS - INRS Ed. - Paris.

- Mathieu N., Schmid AF. (2014) « Modélisation et interdisciplinarité » Quae Ed. - Paris.

- Maziz A., Concas A., Khaldi A., Stålhand J., Persson N.K., Jager E. (2017) "Knitting and weaving artificial muscles" Science Advances, $\underline{3}$, e1600327.

- Mendelssohn F. (1999) cité par Aguerre J.C. «Le champ du vampire » 25-46 in Y. Ferraton Ed. « Le diable en musique » Presses Universitaires de Nancy Ed. - Nancy.

- Ménissier T. (2020) «L'« éleucratie »: et si nous inventions un statut pour protéger la liberté de penser ?» https://theconversation.com/l-eleucratie-et-si-nous-inventions-un-statut-pour-proteger-la-liberte-de-penser149838?utm medium=email\&utm campaign=La\%20lettre\%20de\%20The\%20Conversation\%20France\%20du\%20 24\%20novembre\%202020\%20-

\%201794317418\&utm_content=La\%20lettre\%20de\%20The\%20Conversation\%20France\%20du\%2024\%20novem bre\%202020\%20-

\%201794317418+CID_bea2b9c51acd47ab5b21ac4aaa4f4faf\&utm_source=campaign_monitor_fr\&utm_term=L\%2 Oleucratie\%20\%20\%20et\%20si\%20nous\%20inventions\%20un\%20statut\%20pour\%20protger\%20la\%20libert\%20 de\%20penser

- Mercier A. (2020) «Chercheurs : quels services rendus à la cité ?» https://theconversation.com/chercheurs-quelsservices-rendus-a-la-cite-

132406? utm medium=email\&utm campaign=La\%20lettre\%20de\%20The\%20Conversation \%20France\%20du $\% 20$ $26 \% 20$ fvrier\%202020\%20-

$\% 201546114766 \& u$ tm_content=La\%20lettre\%20de\%20The\%20Conversation $\% 20$ France $\% 20 \mathrm{du} \% 2026 \% 20 \mathrm{fvrier}$ $\% 202020 \% 20-$

\%201546114766+CID d17b4f53a7ae23573ab95cc3a6e540a5\&utm source=campaign monitor fr\&utm term=Ch ercheurs\%20\%20quels\%20services\%20rendus\%20\%201a\%20cit

- MESRI - Ministère de l'Enseignement Supérieur, de l'Industrie et de l'Innovation (2020) « Loi de programmation de la recherche 2021-2030» https://www.enseignementsup-recherche.gouv.fr/pid39124/le-projet-de-loi-deprogrammation-de-la-recherche-2021-2030.html 
- Michéa J.C. (2007) «L’empire du moindre mal » Champs Essais Ed. - Paris.

- Momeni F., Ni J. (2018) “Nature-inspired smart solar concentrators by 4D printing” Renewable Energy, 122, 35-44.

- Momeni F., Ni J. (2020) “Laws of 4D Printing” Engineering, $\underline{6}$, 1035-1055.

- Momeni F., Mehdi Hassani S.M., Liu N.X., Ni J. (2017) “A review of 4D printing” Materials \& Design, 122, 42-79.

- Monod T. (1997) « Terre et ciel » Actes Sud Ed. - Arles.

- Mordor Intelligence (2020) "4D Printing Market - Growth, Trends, and Forecast (2020 - 2025)" https://www.mordorintelligence.com/industry-reports/4d-printing-market

- More T. (1987) «L’Utopie » Flammarion Ed. - Paris.

- Muller-Colard M. (2017) «L'intranquilité » Bayard Ed. - Paris.

- Mynasian G.G. (2018) "Project management in the sphere of innovations: interdisciplinary approach" Vestnik Voronežskogo Gosudarstvennogo Universiteta Inženernyh Tehnologij, 9, 289-296.

- Nakano H. (2010) "Direction control of photomechanical bending of a photochromic molecular fiber" Journal of Material Chemistry, 20, 2071-2074.

- Nal E. (2013) «Réciprocité du croire et circulation du persuader dans la relation d'accompagnement au projet » Nouvelle revue de psychosociologie, $\underline{16}, 215-227$.

- Nguyen D. (2020) « Opinion - Chercheurs, sortez de votre zone de confort : entreprenez ! » https://www.lesechos.fr/idees-debats/cercle/opinion-chercheurs-sortez-de-votre-zone-de-confort-entreprenez1171877

- Nichols M. (2020) "How Is 3D Printing Improving The Manufacturing Industry?" https://www.fabbaloo.com/news/how-is-3d-printing-improving-the-manufacturingindustry?utm_source=newsletter\&utm_medium=email\&utm_campaign=fabbaloo_daily_3d_printing_news\&utm_t $\underline{\mathrm{erm}=2020-11-26}$

- Nisser M.E.W., Felton S.M., Tolley M.T., Rubenstein M., Wood R.J. (2016) "Feedback-Controlled Self-Folding of Autonomous Robot Collectives" IROS 2016 - IEEE/RSJ International Conference on Intelligent Robots and Systems - Daejeon - Corée, 9-14 Octobre 2016, 1254 - 1261.

- NKWR - N.K. Wood Research (2019) "Global 4D Printing Market Forecast 2019-2028" https://www.inkwoodresearch.com/reports/4d-printing-market/

- Novak N., Vesenjak M., Ren Z. (2016) “Auxetic Cellular Materials - a Review” Journal of Mechanical Engineering, $\underline{62}, 485-493$.

- Orwell G. (2008) « A ma guise: Chroniques 1943-1947 » Agone Ed. - Paris.

- Patel P., Pavitt K. (1997) “The Technological Competencies of the Wold's Largest Firms: Complex and PathDependent, But Not Much Variety” Research Policy, 26, 141-156.

- Patel D.K., Sakhaei A.K., Layani M., Zhang B., Ge Q., Magdassi S. (2017) "Highly Stretchable and UV Curable Elastomers for Digital Light Processing Based 3D Printing” Advanced Materials, 1606000.

- Pavitt K. (2005) "Innovation Processes" 86-114 in Fagerberg, J., Movery, D.C., Nelson, R.R. Ed. "The Oxford Handbook of Innovation” Oxford University Press Ed. - Oxford -UK.

- Peach T. (2017) «Insoumissions - Portrait de la France qui vient » Seuil Ed. - Paris.

- Peele B.N., Wallin T.J., Zhao H., Sheperd R.F. (2015) “3D printing antagonistic system of artificial muscle using projection stereo-lithography” Bio-inspiration, Biomimicry, $\underline{10}, 055003$.

- Peguiron F. (2008) «L'intelligence économique au service des acteurs de l'université - La question du partage de l'information sur les campus » L'Harmattan Ed. - Paris - France.

- Pei E., Loh H.G. (2018) "Technological considerations for 4D printing: an overview" Progress in Additive Manufacturing, $\underline{3}$, 95-107 - https://doi.org/10.1007/s40964-018-0047-1

- Petr M., Helgeson M.E., Soulages J., McKinley G.H., Hammond P.T. (2015) "Rapid Viscoelastic Switching of an Ambient Temperature Range Photo-responsive Azo-benzene Side Chain Liquid Crystal Polymer” Polymer, $\underline{54}$, 2850-2856.

- Piaget J. (1967) « Biologie et connaissance » Gallimard Ed. - Paris. 
- Piedrahita-Bello M., Angulo-Cervera J.E., Courson R., Molnar G., Malaquin L., Thibault C., Tondu B., Salmon L., Bousseksou A. (2020) “ 4D printing with spin-crossover polymer composites” Journal of Material Chemistry, C8, 6001-6005.

- PMR - Polaris Market Research (2020) "4D Printing Market Size Worth \$ 419.5 Million by 2026" https://www.polarismarketresearch.com/press-releases/4d-printing-market

- Rafiee M., Farahani R.D., Therriault D. (2020) "Multi-Material 3D and 4D Printing: A Survey" Advanced Sciences, 7, 1902307.

- Rafsanjani A., Bertoldi K., Studart A.R. (2020) "Programming Soft Robots with Flexible Mechanical Metamaterials" https://arxiv.org/ftp/arxiv/papers/1906/1906.00306.pdf

- Rander (2020) “4D Printing: A Revolution across Industries » https://www.machinedesign.com/3d-printingcad/article/21837454/4d-printing-a-revolution-across-industries

- Raymond L. (2000) «Globalisation, économie du savoir et compétitivité: un cadre de veille des tendances et enjeux stratégiques pour les PME » Gestion, 25, 29-38.

- Research and Market (2016) "Global 4D Printing Market to Grow 40\% during 2019-2027" http://www.dailycadcam.com/global-4d-printing-market-to-grow-40-during-2019-2027/

- Roozenburg N., Eckels J. (1995) “Product design: Fundamentals and methods” J. Wiley \& Sons Ed. - New-York USA.

- Rosales A.M., Mabry K.M., Nehls E.M., Anseth K.S. (2015) "Photo-responsive elastic properties of azo-benzenecontaining poly-(ethylene-glycol)-based hydrogels" Bio-macromolecules, $\underline{16}$, 798-806.

- Runco M.A. (1995) “Insight for creativity, expression for impact” Creativity Research Journal, $\underline{8}, 377-390$.

- Runco M.A. (1996) "Personal creativity: Definition and developmental issues" New Directions for Child Development, $\underline{72}, 3-30$.

- Runco M.A. (2015) “Meta-creativity: Being creative about creativity” Creativity Research Journal, 27, 295- 298.

- Runco M.A., Jaeger G. (2012) “The standard definition of creativity” Creativity Research Journal, 24, 92-96.

- Russell M.G., Smorodinskaya N.V. (2018) “Leveraging Complexity for Eco-systemic Innovation” Technological Forecasting and Social Change, 136, 114-131.

- Ryan K.R., Down M.P., Banks C.E. (2020) "Future of Additive Manufacturing: Overview of 4D and 3D Printed Smart and Advanced Materials and their Applications" Chemical Engineering Journal, 126162 https://www.sciencedirect.com/science/article/abs/pii/S1385894720322907

- Sadok M., Benabdallah S., Lesca H. (2003) «Apports Différentiels de l'Internet pour la Veille Anticipative : Application au cas de réponse aux Atteintes à la Sécurité des Réseaux d'entreprises » Actes du 8ième Colloque de l'AIM - Grenoble - France.

- Salgues B., Bister L. (2014) «Le marketing des objets technologiques et des services numériques » ISTE Ed. Londres - UK.

- Samra Y.M., Zhang H., Lynn G.S., Reilly R.R. (2019) “Crisis Management in New Product Development: A Tale of Two Stories" Technovation, $\underline{88}, 102038,1-12$.

- Sarazin B., Cohendet P., Simon L. (2017) «Introduction» 7-11 in « Les communautés d'innovation» EMS Ed. Paris.

- Schaffner M., Faber J.A., Pianegonda L., Rühs P.A., Coulter F, Studart A.R. (2018) "3D printing of robotic soft actuators with programmable bioinspired architectures” Nature Communications, $\underline{9}, 878$.

- Schiffter F. (2019) « Gaston Lagaffe « Philosophie Magazine Ed. - Paris.

- Schwab K. (2017) “The Fourth Industrial Revolution” Penguin Ed. - Random House - USA.

- Shiblee N.I., Ahmed K., Khosla A., Kawakami M., Furukawa H. (2018) "3D printing of shape memory hydrogels with tunable mechanical properties" Soft Matter, 14, 7809-7817.

- Simon H.A. (1976) "From substantive to procedural rationality" 129-148 in Latsis S.J. Ed. "Methods and Appraisal in Economics" Cambridge University Press Ed. - Cambridge - USA.

- Simon H.A. (1995) "Design and systems: General applications of methodology” A. Collen \& W.W. Gasparski Ed. New-Brunswik - USA. 
- Simon H.A. (2004) « Les sciences de l'artificiel » Folio-Essais Ed. - Paris.

- Smith E., Williams-Jones B. (2020) «Lorsque la science fait fausse route: quelques leçons du «Lancetgate »» https://www.latribune.ca/opinions/lorsque-la-science-fait-fausse-route-quelques-lecons-du-lancetgate333d8743b67b5a0e4154a5d4b4f7bf28

- Smith E., Williams-Jones B., Master Z., Larivière V., Sugimoto C.R., Paul-Hus A., Shi M., Resnik D.B. (2020) "Misconduct and Misbehavior Related to Authorship Disagreements in Collaborative Science" Science and Engineering Ethics, 26, 1967-1993.

- Sperber D. (1996) « La contagion des idées » O. Jacob Ed. - Paris.

Stevenson K. (2020) "Who's Working on Volumetric 3D Printing?" https://www.fabbaloo.com/blog/2020/2/25/whos-working-on-volumetric-3d-printing

- Sossou G., Demoly F., Montavon G., Gomes S. (2018) "Design for 4D printing: rapidly exploring the design space around smart materials" Procedia CIRP, 70, 120-125.

- Sossou G., Demoly F., Belkebir H., Qi H.J., Gomes S., Montavon G. (2019) “Design for 4D printing: A voxel-based modeling and simulation of smart materials" Materials \& Design, 175, 107798.

- Sossou G., Demoly F., Belkebir H., Qi H. J., Gomes S., Montavon G. (2019 a) "Design for 4D printing: Modeling and computation of smart materials distributions" Materials \& Design, 181, 108074.

- Stouffer W.B., Russell J.S., Olivia M.G. (2004) "Making the Strange Familiar: Creativity and the Future of Engineering Education" American Society for Engineering, Education Annual Conf. \& Exposition https://pdfs.semanticscholar.org/dd49/3d0c9e6298a8aa0ad17d829a4db8f14f166b.pdf? ga=2.55701928.207523073 $\underline{8.1578563932-1657860172.1578563932}$

- Tang Y., Chi Y., Sun J., Huang T.H., Maghsoudi O.H., Spence A., Zhao J., Su H., Yin J. (2020) "Leveraging elastic instabilities for amplified performance: Spine-inspired high-speed and high-force soft robots" Science Advances, $\underline{8}$, eaaz6912.

- Teoh J.E.M., An J., Chua C.K., Lv M., Krishnasamy V., Liu Y. (2017) "Hierarchically self-morphing structure through 4D printing" Virtual and Physical Prototyping, 12, 61-68.

- Tibbits S. (2013 a) "A very cool new video: A brief introduction to 4D printing" https://blog.ted.com/a-briefintroduction-to-4d-printing/

Tibbits S. (2013) "The emergence of 4D Printing"

https://www.ted.com/talks/skylar_tibbits_the_emergence_of_4d_printing

- Tibbits S. (2014) “4D printing: multi-material shape change” Architecture Design, $\underline{84}, 116-121$.

- Tirole J. (2016) “Economie du bien commun” PUF Ed. - Paris.

- Vallayes F. (2020) « Misère du gouvernement par la peur et la disproportion » https://upmagazine.info/decryptages/analyses/48526-misere-du-gouvernement-par-la-peur-et-la-

disproportion/?utm_source=ActiveCampaign\&utm_medium=email\&utm_content=ACTION\%21+La+newsletter+q uotidienne+de+UP++Magazine\&utm_campaign=ACTION\%21+Quotidienne+lu-2704

- Vielhaber M., Stoffels P. (2014) "Product Development Vs. Production Development” Procedia CIRP, 21, 252-257.

- Vittori J.M. (2020) «Quand les entreprises embaucheront des cœurs » https://www.lesechos.fr/2016/01/quand-lesentreprises-embaucheront-des-coeurs-194055

- VMR - Verified Market Research (2019) "4D Printing Market Size and Forecast" https://www.verifiedmarketresearch.com/product/4d-printing-market/

- VSR - Veracious Statistics Research (2019) "Global Market Growth Opportunities (Revenue, Growth) by 20192026" https://www.veraciousstatisticsresearch.com/research-study/4d-printing-market/

- Walters P., Rossiter J., McGoran J. (2010) "Smart materials and novel actuators: creative applications in art and design"

http://www.uwe.ac.uk/sca/research/cfpr/research/3D/research_projects/smart_materials_novel_actuators.html

- Walton D. (1992) “Slippery slope argument” Clarenton Press Ed. - Oxford - UK. 
- Wang Y., Lee K. (2017) "3D-printed semi-soft mechanisms inspired by origami twisted tower" NASA/ESA Conference on Adaptive Hardware and Systems (AHS) - Pasadena - USA, 2017, 161-166 - doi: 10.1109/AHS.2017.8046373 and (2018) "Three-Dimensional Printable Origami Twisted Tower: Design, Fabrication, and Robot Embodiment" IEEE Robotics and Automation Letters, $\underline{3}, 116$ - 123.

- Weller C., Kleer R., Piller F.T. (2015) "Economic implications of 3D printing: Market structure models in light of additive manufacturing revisited" International Journal of Production Economics, 164, 43-56.

- Wissmann H. (2010) « Au-delà du scientisme » 41-60 in J.M. Besnier, E. Klein, H. Le Guyader, H. Wissmann Ed. « La science en jeu » Actes Sud/IHEST Ed. - Paris.

- Xin X., Liu L., Liu Y., Leng J. (2019) "Mechanical Models, Structures, and Applications of Shape-Memory Polymers and Their Composites" Acta Mechanica Solida Sinica, 32, 535-565.

- Yoshino T., Kondo M., Mamiya J., Kinoshita M. Yu Y.L., Ikeda T. (2010) “Three dimensional photo-mobility of crosslinked azo-benzene liquid crystalline polymer fiber” Advanced Materials, 22, 1361-1363.

- Yu K., Ritchie A., Mao Y., Dunn M.L., Qi H.J. (2015) “Controlled sequential shape changing components by 3D printing of shape memory polymer multi-materials” Procedia IUTAM, 12, 193-203.

- Yu Y., Nakano M., Ikeda T. (2003) "Photo-mechanics: directed bending of a polymer film by light" Nature, $\underline{425}$, 145.

- Zaghloul A., Bone G.M. (2020) «3D Shrinking for Rapid Fabrication of Origami-Inspired Semi-Soft Pneumatic Actuators" IEEE Access, $\underline{8}, 191330$ - 191340.

- Zare M., Prabhakaran M.P., Parvin N., Ramakrishna S. (2019) "Thermally-induced two-way shape memory polymers: Mechanisms, structures, and applications" Chemical Engineering Journal, $\underline{374}$, 706-720.

- Zerbib R., Mamavi O. (2020) «Pourquoi les innovations de rupture sont-elles si difficiles à contrer ?» https://www.hbrfrance.fr/chroniques-experts/2020/11/32418-pourquoi-les-innovations-de-rupture-sont-elles-sidifficiles-a-contrer/

- Zhang L., Liang H., Jacob J., Naumov P. (2015) "Photogated humidity-driven motility" Nature Comm., DOI: 10.1038/ncomms 8429 |www.nature.com/naturecommunications

- Zhang X., Pint C.L., Lee M.H., Scubert B.E., Jamshidi A., Takei K., Ko H., Gillies A.,Bardhan R., Urban J.J.,Wu M., Fearing R., Javey A. (2011) "Optically- and Thermally-Responsive Programmable Materials Based on Carbon Nanotube-Hydrogel Polymer Composites" Nano Letters, 11, 3239-3244.

- Zhang Z., Demir K.G., Gu G.X. (2019) "Developments in 4D-printing: A review on current smart materials, technologies, and applications" International Journal of Smart and Nano Materials, 10, 205-224,

- Zhang Y., Yin X.Y., Zheng M., Moorlag C., Yang J., Wang Z.L. (2019) "3D printing of thermos-reversible polyurethanes with targeted shape memory and precise in situ self-healing properties" Journal of Material Chemistry, $\underline{\text { A7 }}, 6972-6984$.

- Zhao Q., Qi H.J., Xie T. (2015) "Recent progress in shape memory polymer: new behavior, enabling materials, and mechanistic understanding" Progress in Polymer Sci., 49-50, 79-120.

- Ziman J. (2000) "Real Science. What it is, and what it means" Cambridge University Press Ed. - Cambridge - UK.

- Zolfagharian A., Kaynak A., Khoo Y.S., Zhang J., Nahavandi S., Kouzani A. (2019) "Control-oriented modelling of a 3D-printed soft actuator" Materials, 12, 71.

- Zolfagharian A., Kaynak A., Kouzani A. (2020) “Closed-loop 4D-printed soft robots” Materials and Design, 188, 108411.

- Zyla G., Surkamp N., Gurevich E.L., Esen C., Klehr A., Knigge A., Hofmann M.R., Ostendorf A. (2020) "Twophoton polymerization with diode lasers emitting ultrashort pulses with high repetition rate" Optics Letters, $\underline{45}$, 4827-4830.

«Il était une fois un laboureur qui suait sur son champ à quelques lieues de Bagdad. Vint à passer devant lui un homme hideux et inquiétant, vêtu de noir et de mauvais présages. « Qui es-tu étranger ? Où vas-tu ? Que fais-tu ? » Lui demande le paysan. D’une voix étonnamment douce et aimable, l'être sombre lui répond : «Je suis la peste, et m'en vais à Bagdad où je tuerai 500 personnes ». Quelques temps plus tard, s'étant instruit sur les ravages de l'épidémie dans la capitale, le laboureur devise tout à coup l'homme en noir de retour sur le chemin. « Eh, menteur ! 
Pourquoi m'as-tu dit que tu allais en tuer 500 à Bagdad, si l'on déplore plus de 5000 morts ?». Du même ton doux, la peste lui répond : « Je ne t'ai pas menti, et ce n'est pas ma faute. Moi, je n'en ai tué que 500. Les autres sont morts de peur »» (Vallayes, 2020). 